December 11, 2006

hep-ph/0612123

DESY 06-224

$\mathrm{SFB} / \mathrm{CPP}-06-55$

\title{
Two-Loop Renormalization in the Standard Model
}

\section{Part II: Renormalization Procedures and Computational Techniques*}

\author{
Stefano Actis \\ Deutsches Electronen - Synchrotron, DESY, Platanenallee 6, 15738 Zeuthen, Germany \\ Giampiero PASSARINd团 \\ Dipartimento di Fisica Teorica, Università di Torino, Italy \\ INFN, Sezione di Torino, Italy
}

In part I general aspects of the renormalization of a spontaneously broken gauge theory have been introduced. Here, in part II, two-loop renormalization is introduced and discussed within the context of the minimal Standard Model. Therefore, this paper deals with the transition between bare parameters and fields to renormalized ones. The full list of one- and two-loop counterterms is shown and it is proven that, by a suitable extension of the formalism already introduced at the one-loop level, two-point functions suffice in renormalizing the model. The problem of overlapping ultraviolet divergencies is analyzed and it is shown that all counterterms are local and of polynomial nature. The original program of 't Hooft and Veltman is at work. Finite parts are written in a way that allows for a fast and reliable numerical integration with all collinear logarithms extracted analytically. Finite renormalization, the transition between renormalized parameters and physical (pseudo-)observables, will be discussed in part III where numerical results, e.g. for the complex poles of the unstable gauge bosons, will be shown. An attempt will be made to define the running of the electromagnetic coupling constant at the two-loop level.

Key words: Renormalization, Feynman diagrams, Multi-loop calculations,

PACS Classification: 11.10.-z, 11.15.Bt, 12.38.Bx, 02.90.+p, 02.60.Jh, 02.70.Wz

\footnotetext{
${ }^{*}$ Work supported by MIUR under contract 2001023713_006 and by the European Community's Marie-Curie Research Training Network under contract MRTN-CT-2006-035505 'Tools and Precision Calculations for Physics Discoveries at Colliders'.

†Stefano.Actis@desy.de

‡giampiero@to.infn.it
} 


\section{Contents}

1 Introduction 1

2 Notation and conventions $\quad 2$

3 Outline of the calculation 2

3.1 Ultraviolet decompositions . . . . . . . . . . . . . . . . . . . . . . . . . . . . 4

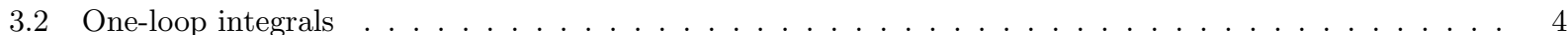

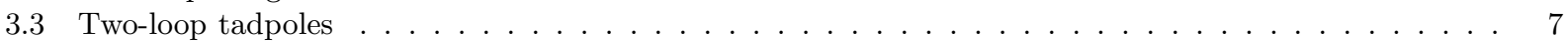

3.4 Two-loop two-point integrals . . . . . . . . . . . . . . . . . . . . . . 8

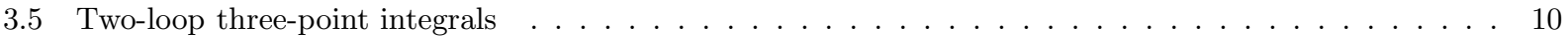

$4 \overline{M S}$ renormalization at two loops

4.1 Gauge fixing . . . . . . . . . . . . . . . . . . . . . . . . . . 12

4.2 Definition of the renormalization scheme: $\overline{M S}$ and beyond . . . . . . . . . . . . . . . . . 14

5 One-loop renormalization $\mathbf{1 6}$

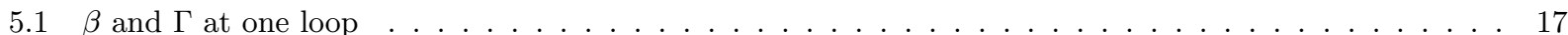

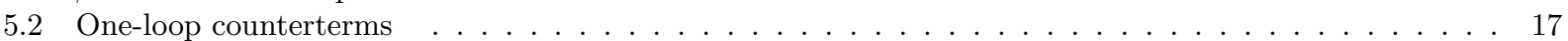

5.3 Finite renormalization . . . . . . . . . . . . . . . . . . . . . . . . 18

5.4 Ward-Slavnov-Taylor identities . . . . . . . . . . . . . . . . . . . . . . . 21

$6 \quad \beta$ and $\Gamma$ at two loops 23

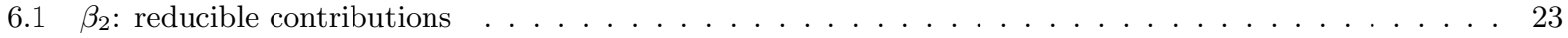

$6.2 \beta_{2}$ : irreducible contributions . . . . . . . . . . . . . . . . . . . . . . . 24

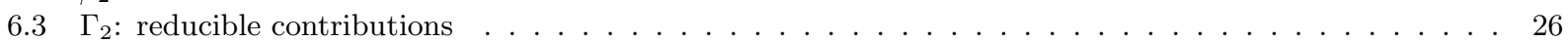

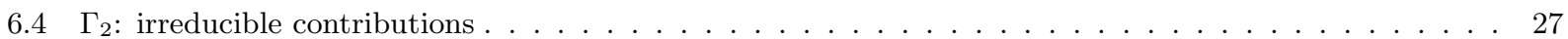

7 Two-loop self-energies $\mathbf{2 8}$

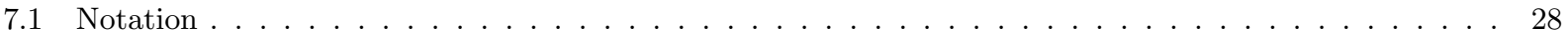

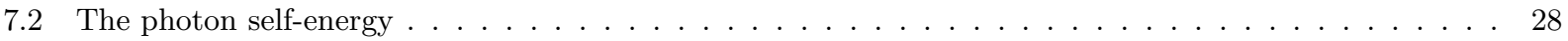

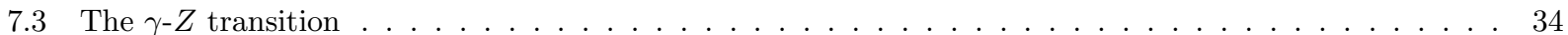

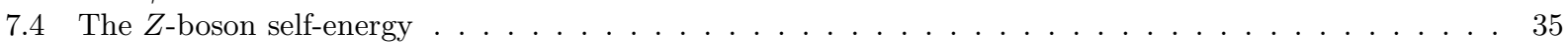

7.5 The $W$-boson self-energy $\ldots \ldots \ldots \ldots \ldots \ldots \ldots$

7.6 The Higgs-boson self-energy . . . . . . . . . . . . . . . . . . . . . . 37

8 Two-Loop Counterterms

9 Conclusions 40

A Reduction of vacuum bubbles 43

B Singularities whose origin is infrared 43

B.1 Evaluation of $s^{C}\left(s=0 ; m_{1}, m_{2}, 0,0\right) \ldots \ldots \ldots \ldots$

B.2 Evaluation of $s^{E}\left(s, m_{1}, m_{2}, 0, m_{4}, 0\right) \ldots \ldots \ldots \ldots$

C The limit $m_{f} \rightarrow 0$

D The limit $p^{2} \rightarrow 0 \quad 48$

D.1 One-loop functions . . . . . . . . . . . . . . . . . . . . . . . . . . . . . 48

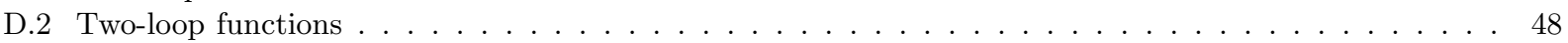

D.3 Limit $m_{f} \rightarrow 0$ of $p^{2} \rightarrow 0 \ldots \ldots \ldots \ldots \ldots$

E Finite mass renormalization in $\Pi^{R} \quad 53$ 


\section{Introduction}

After the end of the Lep era it became evident that including estimates of higher-order radiative corrections into one-loop calculations for physical (pseudo-)observables could not satisfy anymore the need for precision required by the forthcoming generation of experiments. Since LHC will be an arena for discovery physics, high precision will not be mandatory during its first phase. However, according to some predestinate design, hadron machines are alternating with electron-positron ones and, hopefully, ILC will come into operation: here the highest available theoretical precision will play a fundamental role.

As a matter of fact, it is not clear what kind of scenario will arise after the first few months of running at LHC. Any evidence of new physics will lead to a striking search for new theoretical models and their Born predictions; the quake could be so strong to remove any interest in quantum effects of the Standard Model. On the contrary, we could be back to the familiar landscape: effects of new physics hidden inside loops.

Since we have no firm opinion, we decided to follow the old rule si vis pacem para bellum, building the environment that allows for a complete two-loop analysis of a spontaneously broken gauge field theory. This construction requires several elements, and it is difficult to characterize our strategy with a single acronym; although our work implies a lot of analytical aspects, the final step (computing arbitrary two-loop diagrams) can only be done with an algebraic-numerical approach.

If one thinks for a while, everything is in the old papers of 't Hooft and Veltman [1, but translating few formal properties into a working scheme is far from trivial. Most of the times it is not a question of How do I do it?, rather it is a question of bookkeeping: Can I do it without exhausting the memory of my computer?, or, Is there any practical way of presenting my results besides making my codes public?.

We devoted a first paper 2] (hereafter I) to deal with general aspects of a spontaneously broken gauge theory. First of all, we showed how to treat tadpoles; although everybody knows how to do it, general results are rarely presented in a way that everyone can use them. In addition, we analyzed how to perform an order-by-order diagonalization of the neutral sector of a theory of fundamental interactions; once again, one needs a comprehensive collection of results which allows for practical applications.

Alternative solutions to solving these problems exist, noticeably in the background-field formalism [3] (compare also with Ref. 4]); our claim here is restricted to the construction of a set of procedures which do not rely on other sources and cover broadly all the aspects, from generation of diagrams and renormalization to evaluation (mostly numerical) of physical processes. In particular, our results show that the structure of the counterterms at the two-loop level, as well as of the whole set of renormalized Green functions and the Ward-Slavnov-Taylor identities [5, has (in the conventional approach) a degree of simplicity comparable to the one obtained at one loop in the background-field approach.

Another perennial question is: what about renormalization, with or without counterterms? In a way, it is a fake question, since the two approaches are fully equivalent as far as $S$-matrix elements are concerned. In this paper we focus on the transition from bare parameters to renormalized ones, and in a third paper (hereafter III) we will then discuss the ultimate step in any renormalization procedure: the transition from renormalized parameters to a set of physical (pseudo-)observables. Perhaps, one should try to make a clear vocabulary of renormalization in quantum field theory. A renormalization procedure is designed to bring you from a Lagrangian to theoretical predictions; it includes regularization (nowadays dimensional regularization [1] is easy to understand), a renormalization scheme and the choice of an input parameter set. The scheme, being a transitory step, is almost irrelevant; it can be on-mass-shell or $\overline{M S}$ or based on complex poles, but unless you do something illegal (resummations that are not allowed or similar things) it really does not matter. Admittedly, one can define $\overline{M S}$ quantities as convenient tools, but it is the last step that matters, at least as long as we have a convenient subtraction point (which we miss in QCD).

Renormalized quantities should always be expressed in terms of a set of physical quantities. One may indulge to the introduction of an $\overline{M S}$ running electromagnetic coupling constant (importing a concept from QCD to QED, which sounds strange anyway) but, at the end of the day, only cross sections matter.

In this paper, we have done one thing: all the two-loop Green's functions of the theory are made finite by introducing non-logarithmic counterterms and respecting unitarity. In addition, one can easily check that renormalized Ward-Slavnov-Taylor identities are satisfied. Actually, we have done more, since all ultravioletfinite parts have been classified and an algorithm has been designed for their evaluation at any scale. What 
is innovative in our approach, as well as in other modern approaches $\underline{6}$, is the idea that everything can be generated (is generated) by a set of automatized procedures which deals satisfactorily with the somewhat greater complexity of a two-loop calculation.

Furthermore, classification of ultraviolet divergencies is dynamically linked to a well-defined computational scheme. In other words, in our approach, the ultraviolet-finite parts are back in their privileged position where they can play the role of creating the predictive power of the theory. There are, of course, preliminary steps (not always the easy ones), but it is only the full control on the multi-scale level that pays off.

The outline of this paper is as follows. After introducing our notation and conventions in Section 2 we outline the strategy of our calculation in Section 3, where we classify all the ultraviolet-divergent parts of the needed loop integrals. Next, in Section 4, we choose the gauge-fixing Lagrangian and we define our renormalization scheme. In Section 5 we review one-loop renormalization, and in Section 6 we show explicit results for tadpole renormalization and neutral-sector diagonalization at two loops. Finally, we analyze the ultraviolet structure of two-loop self-energies in Section 7 and we show explicit results for the two-loop counterterms in Section 8. Section 9 contains the summary, and several technical details connected with the relevant kinematical limits, which represent the backbone of our computational techniques, are discussed in the appendices.

\section{Notation and conventions}

Regularization. We employ dimensional regularization [1, denoting the number of the space-time dimensions by $n=4-\epsilon$. In addition, we use a short-hand notation for regularization-dependent factors,

$$
\Delta_{U V}=\gamma+\ln \pi+\ln \frac{M^{2}}{\mu^{2}}, \quad \Delta_{U V}(x)=\Delta_{U V}-\ln \frac{M^{2}}{x},
$$

where $\gamma=0.5772156 \cdots$ is the Euler constant, $\mu$ is the 't Hooft unit of mass, $M$ stands for the bare (renormalized) $W$-boson mass (we do not distinguish unless strictly needed) and $x$ is a positive-definite kinematical variable. In our conventions the logarithm has a cut along the negative real axis and it is understood that for all masses: $M^{2} \rightarrow M^{2}-i \delta$, with $\delta \rightarrow 0_{+}$.

Masses. We introduce a compact notation for ratios of squared masses,

$$
x_{H}=\frac{M_{H}^{2}}{M^{2}}, \quad x_{l, i}=\frac{m_{l, i}^{2}}{M^{2}}, \quad x_{u, i}=\frac{m_{u, i}^{2}}{M^{2}}, \quad x_{d, i}=\frac{m_{d, i}^{2}}{M^{2}} .
$$

Here $M_{H}$ is the Higgs-boson bare (renormalized) mass and $m_{l, i}, m_{u, i}$ and $m_{d, i}$ are the bare (renormalized) masses of the charged lepton and the up and down quarks of the $i$ th fermion doublet, with $i=1, \ldots, 3$. We consider the minimal representation for the Standard-Model scalar sector, defining $M_{0}=M / c_{\theta}$ for the $Z$-boson bare (renormalized) mass. Here $c_{\theta}\left(s_{\theta}\right)$ is the bare or renormalized cosine (sine) of the weak-mixing angle $\theta$. For notational clarity we frequently employ the notation

$$
\{m\}_{12 \ldots N}=m_{1}, m_{2}, \ldots, m_{N} .
$$

\section{Outline of the calculation}

Our calculation builds upon an automatic strategy for generating Feynman diagrams and evaluating the necessary one- and two-loop integrals. In addition, it does not rely on any black-box tool: diagrams are generated through a set of FORM [7] routines implemented in the $\mathcal{G}$ raphShot package [8] and loop integrals are computed through the FORTRAN/95 LoopBack 9 code.

The present work uses a set of results derived in a series of previous papers. The general strategy for handling multi-loop multi-leg Feynman diagrams was designed in Ref. [10] and the whole body of results necessary for evaluating two-loop two-point integrals can be found in Ref. [11. The calculation of two-loop 
three-point scalar integrals is considerably more involved: infrared-convergent configurations are discussed in Ref. 12 and infrared- and collinear-divergent ones are analyzed in Ref. 13. Finally, our method for dealing with two-loop tensor integrals can be found in Ref. [14] and results for one-loop multi-leg integrals are shown in Ref. [15].

It is worth noting that in any spontaneously-broken gauge theory the large number of masses and external kinematical variables leads to difficulties for the familiar analytical methods. Therefore, we use an alternative approach (mostly numerical) which, however, does not imply a blind rejecting of analytical techniques; for instance, in this work, we devote a special attention to the analytical extraction of leading and sub-leading collinear logarithms, since they play a dominant role in any calculation [16].

If we focus on renormalization, we can safely state that all the necessary ingredients are available. Here the crucial point is to connect a set of input experimental data (an input-parameter set, hereafter IPS) to the free parameters of the theory:

- mass renormalization involves the calculation of self-energies [10]11;

- renormalization of coupling constants requires additional elements, which depend on the choice of the (pseudo-)observables (hereafter POs) in the IPS.

The most-obvious selection of an IPS is based on the choice of those data which are known with the best experimental precision: the electromagnetic coupling constant, $\alpha$, and the Fermi coupling constant, $G_{F}$. ¿From the diagrammatic point of view, the whole set of renormalization equations (including finite parts) requires the evaluation of two-loop vertex and box integrals, since $\alpha$ is defined by means of the Thomsonscattering amplitude and $G_{F}$ is extracted from the muon lifetime. However, it is possible to show that:

- neglecting $\mathcal{O}\left(m_{\mu}^{2} / M^{2}\right)$ terms, where $m_{\mu}$ is the muon mass, the evaluation of the muon lifetime can be carried on through tadpole diagrams (zero external momenta);

- the relevant Ward-Slavnov-Taylor (hereafter WST) identities of the Standard Model imply a set of algebraic cancellations involving vertex diagrams and wave-function renormalization factors for the external legs. Here the role played by the parameter $\Gamma$ introduced in I (all-order diagonalization of the neutral sector) plays an essential role. As a result, one can write an electric-charge renormalization equation which depends only on the photon vacuum-polarization function, as in QED.

We defer a complete discussion of this computation to a forthcoming paper (hereafter III); for a short review, see Ref. [17. For the present purposes, it is enough to say that the results collected in Refs. [10[11] allow to complete the whole renormalization program. Of course, properties of two-loop vertex diagrams have to be checked, and here we use the results of Refs. [12 13]14.

An important remark concerns tensor loop integrals, namely those configurations with a non-trivial spin structure for the numerators. On the one hand, they can be reduced to scalar integrals if one uses the standard methods of Ref. 18, supplemented by the sub-loop techniques of Ref. 19. It is worth noting that a complete scalarization for two-loop vertex diagrams requires the introduction of generalized scalar integrals, to be evaluated in shifted space-time dimensions (the corresponding propagators are raised to non-canonical powers). On the other hand, the number of terms generated by the reduction procedure increases rapidly in any realistic computation.

In order to carry out a systematic check of our computation, we have used both approaches: a reduction procedure followed by an extraction of the ultraviolet (hereafter UV) resides of the poles for the scalar configurations and a direct computation of the UV divergencies for the tensor cases. Reduction techniques can introduce negative powers of Gram determinants, which can be dangerous for the numerical stability of the results. The approach we take in the present paper avoids these problems since scalarization is only employed to prove that the relevant algebraic properties are satisfied.

One of the main goals of this paper is to show that UV divergencies at two loops can be removed through suitable polynomial subtraction terms. Therefore, in this section we collect the results for the residues of the UV poles of all the relevant one- and two-loop integrals employed in our calculation. However, we stress that only a full control over the (less-trivial) UV-finite parts will finally allow for theoretical predictions. In Subsection 3.1 we introduce general UV decompositions for one- and two-loop integrals. In Subsection 3.2 
we present the relevant results for the one-loop ingredients. In Subsections 3.3, 3.4 and 3.5 we show the necessary results for one-, two- and three-point two-loop integrals.

\subsection{Ultraviolet decompositions}

One-loop integrals. Any one-loop integral $f^{1}$ can be decomposed as 1

$$
f^{1}(\{l\})=\sum_{k=-1}^{1} f^{1}(\{l\} ; k) F_{k}^{1}(x)
$$

where $\{l\}$ denotes a given set of arguments: powers of the inverse propagators, external kinematical variables, masses of internal particles. $x$ is some kinematical variable (usually $M$ for tadpoles or a squared external momentum) and the dependence on the dimensional regulator $\epsilon$ and the regularization-dependent factors introduced in Eq.(11) is entirely transferred to the universal UV factors,

$$
F_{-1}^{1}(x)=\frac{1}{\epsilon}-\frac{1}{2} \Delta_{U V}(x)+\frac{1}{8} \Delta_{U V}^{2}(x) \epsilon, \quad F_{0}^{1}(x)=1-\frac{1}{2} \Delta_{U V}(x) \epsilon, \quad F_{1}^{1}(x)=\epsilon .
$$

It is worth noting that, because of overlapping divergencies (UV-divergent one-loop sub-diagrams), we include $\mathcal{O}(\epsilon)$ terms in all one-loop results.

Two-loop integrals. A generic two-loop integral $f^{2}$ can be written as

$$
f^{2}(\{l\})=\sum_{k=-2}^{0} f^{2}(\{l\} ; k) F_{k}^{2}(x) .
$$

Here the two-loop UV factors read as follows:

$$
F_{-2}^{2}(x)=\frac{1}{\epsilon^{2}}-\frac{\Delta_{U V}(x)}{\epsilon}+\frac{1}{2} \Delta_{U V}^{2}(x), \quad F_{-1}^{2}(x)=\frac{1}{\epsilon}-\Delta_{U V}(x), \quad F_{0}^{2}(x)=1 .
$$

Note that the product of two one-loop integrals can be written through the same UV decomposition of a two-loop integral. Finally, in the following we collect two overall factors for one- and two-loop integrals,

$$
\mu_{1}(\epsilon)=\frac{\mu^{\epsilon}}{i \pi^{2}}, \quad \mu_{2}(\epsilon)=\frac{\mu^{2 \epsilon}}{\pi^{4}}=-\mu_{1}^{2}(\epsilon)
$$

\subsection{One-loop integrals}

\section{Tadpoles}

A scalar one-loop tadpole, with the inverse propagator raised to an integer power $\alpha$, is defined by

$$
A_{0}(\alpha, m)=\mu_{1}(\epsilon) \int \frac{d^{n} q}{\left(q^{2}+m^{2}\right)^{\alpha}} .
$$

Integration-by-parts (IBP) identities [20] allow to reduce any configuration with $\alpha>1$ to the case $\alpha=1$ by means of a recursive application of the identity

$$
A_{0}(\alpha, m)=-\frac{1}{\alpha-1}\left(\frac{n}{2}-\alpha+1\right) \frac{A_{0}(\alpha-1, m)}{m^{2}} .
$$

We factorize everywhere the scale dependence in order to deal with dimensionless loop integrals. Here we introduce the function $a_{0}(\alpha, m)$,

$$
A_{0}(\alpha, m)=\left(m^{2}\right)^{2-\alpha} a_{0}(\alpha, m),
$$

\footnotetext{
${ }^{1}$ The UV decompositions of Eqs. (4)-(7) and the corresponding classification of UV poles are due to S. Uccirati.
} 
and apply the UV decomposition defined in Eq.(4) to the case $a_{0}(m)=a_{0}(1, m)$,

$$
a_{0}(m)=\sum_{k=-1}^{1} a_{0}(m ; k) F_{k}^{1}\left(M^{2}\right)
$$

The coefficients of the UV factors in Eq.(12) read as

$$
\begin{aligned}
a_{0}(m ;-1) & =-2, \quad a_{0}(m ; 0)=-1+\ln \frac{m^{2}}{M^{2}} \\
a_{0}(m ; 1) & =\frac{1}{2}\left[-1-\frac{1}{2} \zeta(2)+\left(1-\frac{1}{2} \ln \frac{m^{2}}{M^{2}}\right) \ln \frac{m^{2}}{M^{2}}\right],
\end{aligned}
$$

where $\zeta(z)$ is the Riemann zeta function.

\section{Two-point integrals}

Ultraviolet decompositions. For two-point integrals we introduce $s=-p^{2}$, where $p$ is the external momentum. We extract form factors for tensor configurations and introduce scaled quantities according to the following definitions:

$$
\begin{aligned}
B_{\mu \nu}\left(\alpha, \beta, s,\{m\}_{12}\right) & =\mu_{1}(\epsilon) \int d^{n} q \frac{q_{\mu} q_{\nu}}{\left(q^{2}+m_{1}^{2}\right)^{\alpha}\left[(q+p)^{2}+m_{2}^{2}\right]^{\beta}} \\
& =s^{2-\alpha-\beta} b_{21}\left(\alpha, \beta, s,\{m\}_{12}\right) p_{\mu} p_{\nu}+s^{3-\alpha-\beta} b_{22}\left(\alpha, \beta, s,\{m\}_{12}\right) \delta_{\mu \nu}, \\
B_{\mu}\left(\alpha, \beta, s,\{m\}_{12}\right) & =\mu_{1}(\epsilon) \int d^{n} q \frac{q_{\mu}}{\left(q^{2}+m_{1}^{2}\right)^{\alpha}\left[(q+p)^{2}+m_{2}^{2}\right]^{\beta}}=s^{2-\alpha-\beta} b_{1}\left(\alpha, \beta, s,\{m\}_{12}\right) p_{\mu}, \\
B_{0}\left(\alpha, \beta, s,\{m\}_{12}\right) & =\mu_{1}(\epsilon) \int \frac{d^{n} q}{\left(q^{2}+m_{1}^{2}\right)^{\alpha}\left[(q+p)^{2}+m_{2}^{2}\right]^{\beta}}=s^{2-\alpha-\beta} b_{0}\left(\alpha, \beta, s,\{m\}_{12}\right) .
\end{aligned}
$$

Note that scaled one-loop form factors will often appear in the residues of the UV poles for two-loop integrals. Reduction of higher-rank form factors to the scalar case can be obtained by means of well-established techniques. UV decompositions read as

$$
b_{i}\left(\alpha, \beta, s,\{m\}_{12}\right)=\sum_{k=-1}^{1} b_{i}\left(\alpha, \beta, s,\{m\}_{12} ; k\right) F_{k}^{1}(s),
$$

where $i=0,1,21,22$. The coefficients of the UV factors for the canonical scalar case $(\alpha=\beta=1)$ read as

$$
\begin{aligned}
b_{0}\left(1,1, s,\{m\}_{12} ;-1\right) & =2, \quad b_{0}\left(1,1, s,\{m\}_{12} ; 0\right)=b_{0}^{\text {fin }}\left(1,1, s,\{m\}_{12}\right), \\
b_{0}\left(1,1, s,\{m\}_{12} ; 1\right) & =\frac{1}{4}\left[\zeta(2)-b_{0}^{\epsilon}\left(1,1, s,\{m\}_{12}\right)\right] .
\end{aligned}
$$

We will need to consider additional cases, where one propagator is raised to a non-canonical power, i.e. $\alpha=2, \beta=1$. Here results are given by

$$
\begin{aligned}
b_{0}\left(2,1, s,\{m\}_{12} ;-1\right) & =0, \quad b_{0}\left(2,1, s,\{m\}_{12} ; 0\right)=b_{0}^{\text {fin }}\left(2,1, s,\{m\}_{12}\right), \\
b_{0}\left(2,1, s,\{m\}_{12} ; 1\right) & =-\frac{1}{4} b_{0}^{\epsilon}\left(2,1, s,\{m\}_{12}\right) .
\end{aligned}
$$

Note that Eq. (17) holds strictly only when $m_{1} \neq 0$, otherwise an infrared (IR) divergency arises and we need a different decomposition. The finite parts and the $\mathcal{O}(\epsilon)$ terms introduced in Eqs.(16) and (17) have the following integral representations:

$$
\begin{aligned}
& b_{0}^{\mathrm{fin}}\left(1,1, s,\{m\}_{12}\right)=-\int_{0}^{1} d x \ln \frac{\chi(x)}{s}, \quad b_{0}^{\mathrm{fin}}\left(2,1, s,\{m\}_{12}\right)=\int_{0}^{1} d x \frac{(1-x) s}{\chi(x)}, \\
& b_{0}^{\epsilon}\left(1,1, s,\{m\}_{12}\right)=-\int_{0}^{1} d x \ln ^{2} \frac{\chi(x)}{s}, \quad b_{0}^{\epsilon}\left(2,1, s,\{m\}_{12}\right)=2 \int_{0}^{1} d x \frac{(1-x) s}{\chi(x)} \ln \frac{\chi(x)}{s},
\end{aligned}
$$


where the quadratic form $\chi$ reads as

$$
\chi(x)=s x^{2}-\left(s+m_{1}^{2}-m_{2}^{2}\right) x+m_{1}^{2}-i \delta .
$$

Finite components. For the finite parts we compute explicitly the integrals introduced in Eq.(18). For the case $\alpha=\beta=1$ we obtain

$$
\begin{aligned}
b_{0}^{\mathrm{fin}}(1,1, s, 0,0) & =2-L_{\mathrm{an}}, \\
b_{0}^{\mathrm{fin}}(1,1, s, 0, m) & =-\ln \frac{m^{2}}{s}+2-\left(1-\frac{m^{2}}{s}\right) \ln \left(1-\frac{s}{m^{2}}\right), \\
b_{0}^{\mathrm{fin}}(1,1, s, m, m) & =-\ln \frac{m^{2}}{s}+2-\beta\left(\frac{m^{2}}{s}\right) \ln \frac{\beta\left(m^{2} / s\right)+1}{\beta\left(m^{2} / s\right)-1}, \\
b_{0}^{\text {fin }}\left(1,1, s, m_{1}, m_{2}\right) & =-\frac{1}{2}\left[\ln \frac{m_{1}^{2}}{s}+\ln \frac{m_{2}^{2}}{s}+\frac{m_{1}^{2}-m_{2}^{2}}{s}\left(\ln \frac{m_{1}^{2}}{s}-\ln \frac{m_{2}^{2}}{s}\right)\right] \\
& +2-\frac{1}{s} \lambda^{1 / 2}\left(s, m_{1}^{2}, m_{2}^{2}\right) L\left(s, m_{1}^{2}, m_{2}^{2}\right),
\end{aligned}
$$

where $\lambda(x, y, z)$ is the usual Källen function and we introduced

$$
\begin{aligned}
\beta^{2}\left(\frac{m^{2}}{s}\right) & =1-4 \frac{m^{2}}{s+i \delta}, \\
L\left(s, m_{1}^{2}, m_{2}^{2}\right) & =\ln \frac{-s+m_{1}^{2}+m_{2}^{2}-\lambda^{1 / 2}\left(s, m_{1}^{2}, m_{2}^{2}\right)}{2 m_{1} m_{2}}, \\
L_{\text {an }} & =\ln (-1-i \delta), \quad \delta \rightarrow 0_{+} .
\end{aligned}
$$

For the regular $\left(m_{1} \neq 0\right)$ case, where $\alpha=2$ and $\beta=1$, we obtain

$$
\begin{aligned}
b_{0}^{\mathrm{fin}}(2,1, s, m, 0) & =-\ln \left(1-\frac{s}{m^{2}}\right), \\
b_{0}^{\mathrm{fin}}(2,1, s, m, m) & =-\frac{1}{\beta\left(m^{2} / s\right)} \ln \frac{\beta\left(m^{2} / s\right)+1}{\beta\left(m^{2} / s\right)-1}, \\
b_{0}^{\mathrm{fin}}\left(2,1, s, m_{1}, m_{2}\right) & =\frac{m_{1}^{2}-m_{2}^{2}-s}{\lambda^{1 / 2}\left(s, m_{1}^{2}, m_{2}^{2}\right)} L\left(s, m_{1}^{2}, m_{2}^{2}\right)+\frac{1}{2}\left(\ln \frac{m_{1}^{2}}{s}-\ln \frac{m_{2}^{2}}{s}\right) .
\end{aligned}
$$

Infrared-divergent configurations. Two special cases requires a separate discussion: when $m_{1}=0$ in Eq.(14) and $\alpha=2$ or $\beta=1$, an IR divergency shows up. Here we regularize IR divergencies by setting $n=4+\hat{\epsilon}$, and then we use the relation between the UV regulator $\epsilon$ and the IR one $\hat{\epsilon}$, i.e. $\epsilon=-\hat{\epsilon}$. The results read as follows:

$$
\begin{aligned}
b_{0}(2,1, s, 0,0) & =\frac{2}{\epsilon}-\Delta_{U V}(s)-L_{\mathrm{an}}+\frac{\epsilon}{4}\left[\Delta_{U V}^{2}(s)+2 \Delta_{U V}(s) L_{\mathrm{an}}+L_{\mathrm{an}}^{2}-\zeta(2)\right], \\
b_{0}(2,1, s, 0, m) & =\frac{s}{s-m^{2}}\left\{\frac{2}{\epsilon}-\Delta_{U V}(s)-\ln \frac{m^{2}}{s}-\left(1+\frac{m^{2}}{s}\right) \ln \left(1-\frac{s}{m^{2}}\right)\right. \\
& +\frac{\epsilon}{2}\left[\frac{1}{2} \zeta(2)+\frac{1}{2} \Delta_{U V}^{2}(s)+\ln \frac{m^{2}}{s}\left(\Delta_{U V}(s)+\frac{1}{2} \ln \frac{m^{2}}{s}\right)\right. \\
& +\left(1+\frac{m^{2}}{s}\right)\left(\ln \left(1-\frac{s}{m^{2}}\right) \Delta_{U V}(s)+\ln \frac{m^{2}}{s} \ln \left(1-\frac{s}{m^{2}}\right)\right. \\
& \left.\left.\left.+\frac{1}{2} \ln ^{2}\left(1-\frac{s}{m^{2}}\right)-\operatorname{Li}_{2}\left(\frac{s}{s-m^{2}}\right)\right)\right]\right\} .
\end{aligned}
$$

Here we used generalized Nielsen polylogarithms,

$$
S_{n, p}(z)=\frac{(-1)^{n+p-1}}{(n-1) ! p !} \int_{0}^{1} \frac{d x}{x} \ln ^{n-1} x \ln ^{p}(1-z x), \quad S_{n-1,1}(z)=\operatorname{Li}_{n}(z) .
$$




\section{Three-point integrals}

Ultraviolet decompositions. For three-point integrals we extract form factors for tensor configurations and then we introduce scaled quantities. Our notation for a generic tensor structure with $l$ Lorentz indices is

$$
C_{\mu_{1} \ldots \mu_{l}}\left(\alpha_{1}, \alpha_{2}, \alpha_{3}, p_{1}, p_{2}, P,\{m\}_{123}\right)=\mu_{1}(\epsilon) \int d^{n} q \frac{q_{\mu_{1}} \ldots q_{\mu_{l}}}{\left(q^{2}+m_{1}^{2}\right)^{\alpha_{1}}\left[\left(q+p_{1}\right)^{2}+m_{2}^{2}\right]^{\alpha_{2}}\left[(q+P)^{2}+m_{3}^{2}\right]^{\alpha_{3}}},
$$

where $P=p_{1}+p_{2}$. Scaled quantities read as (we omit list of arguments)

$$
\begin{aligned}
C_{\mu \nu} & =s^{2-\alpha_{1}-\alpha_{2}-\alpha_{3}}\left[c_{21} p_{1 \mu} p_{1 \nu}+c_{22} p_{2 \mu} p_{2 \nu}+c_{23}\left\{p_{1} p_{2}\right\}_{\mu \nu}\right]+s^{3-\alpha_{1}-\alpha_{2}-\alpha_{3}} c_{24} \delta_{\mu \nu}, \\
C_{\mu} & =s^{2-\alpha_{1}-\alpha_{2}-\alpha_{3}}\left(c_{11} p_{1 \mu}+c_{12} p_{2 \mu}\right), \quad C_{0}=s^{2-\alpha_{1}-\alpha_{2}-\alpha_{3}} c_{0},
\end{aligned}
$$

where we have also introduced the symmetrized combination $\{p k\}_{\mu \nu}=p_{\mu} k_{\nu}+p_{\nu} k_{\mu}$. Three-point, scaled, one-loop form factors will often appear in the residues of the UV poles for two-loop vertex integrals. Reduction of higher-rank form factors to the scalar case can be obtained by means of standard methods, and UV decompositions read as

$$
c_{i}\left(\alpha_{1}, \alpha_{2}, \alpha_{3}, p_{1}, p_{2}, P,\{m\}_{123}\right)=\sum_{k=-1}^{1} c_{i}\left(\alpha_{1}, \alpha_{2}, \alpha_{3}, p_{1}, p_{2}, P,\{m\}_{123} ; k\right) F_{k}^{1}(s),
$$

where $i$ contains the information on the spin structure and $s=-P^{2}$. Examples of UV decomposition are

$$
c_{0}\left(1,1,1, p_{1}, p_{2}, P,\{m\}_{123} ;-1\right)=0, \quad \ldots \quad c_{24}\left(1,1,1, p_{1}, p_{2}, P,\{m\}_{123} ;-1\right)=\frac{1}{2}, \quad \text { etc. }
$$

\subsection{Two-loop tadpoles}

A special class of two-loop integrals is represented by tadpoles, defined by

$$
T_{i j k}\left(\{m\}_{123}\right)=\mu_{2}(\epsilon) \int \frac{d^{n} q_{1} d^{n} q_{2}}{\left(q_{1}^{2}+m_{1}^{2}\right)^{i}\left(q_{2}^{2}+m_{2}^{2}\right)^{j}\left[\left(q_{1}-q_{2}\right)^{2}+m_{3}^{2}\right]^{k}} .
$$

Any two-loop tadpole can be reduced to a single two-loop master tadpole (we choose the configuration with $i=k=1, j=2$ ) and to products of one-loop tadpoles through iterated application of IBP identities. A few examples are given in Appendix A, The UV decomposition for the master tadpole reads as

$$
T_{121}\left(\{m\}_{123}\right)=\sum_{k=-2}^{0} T_{121}\left(\{m\}_{123} ; k\right) F_{k}^{2}\left(M^{2}\right),
$$

where the coefficients of the UV-divergent factors are given by

$$
T_{121}\left(\{m\}_{123} ;-2\right)=-2, \quad T_{121}\left(\{m\}_{123} ;-1\right)=-\left(1-2 \ln \frac{m_{2}^{2}}{M^{2}}\right) .
$$

These relations hold if $m_{2} \neq 0$, since a collinear logarithm is present when $m_{2} \rightarrow 0$. When $m_{2}=0$ we can change integration variables and apply again IBP identities in order to use Eq.(31), i.e.

$$
T_{121}\left(m_{1}, 0, m_{3}\right)=T_{112}\left(m_{1}, m_{3}, 0\right) \underset{I B P}{\underset{\longrightarrow}{\longrightarrow}} T_{121}\left(m_{1}, m_{3}, 0\right)
$$

and the $\left(m_{1}, m_{3}, 0\right)$ configuration, which is collinear-free, can be computed as shown in Eq.(223) of Appendix $\mathrm{A}$. 


\subsection{Two-loop two-point integrals}

Notation. We follow the notation of Section 7 of Ref. 14] and we introduce scaled irreducible two-loop two-point integrals. Note that when the squared external momentum vanishes, $p^{2}=0$, the tadpole-reduction methods of Subsection 3.3 can be easily employed. Otherwise, we extract $s=-p^{2}$ and accordingly define

$$
S_{0}^{A}=s s_{0}^{A}, \quad S_{0}^{C}=s_{0}^{C}, \quad S_{0}^{D}=\frac{1}{s} s_{0}^{D}, \quad S_{0}^{E}=\frac{1}{s} s_{0}^{E} .
$$

The alphameric classification of graphs can be found in Subsection 2.2 of Ref. 14. For tensor configurations we employ form factors according to Section 7 of Ref. [14,

$$
\begin{aligned}
\operatorname{rank} 1 \rightarrow S^{I}(\mu \mid 0) & =S_{1}^{I} p_{\mu}, & S^{I}(0 \mid \mu)=S_{2}^{I} p_{\mu}, \\
\operatorname{rank} 2 \rightarrow \quad S^{I}(\mu, \nu \mid 0) & =S_{112}^{I} \delta_{\mu \nu}+S_{111}^{I} p_{\mu} p_{\nu}, & S^{I}(\mu \mid \nu)=S_{122}^{I} \delta_{\mu \nu}+S_{121}^{I} p_{\mu} p_{\nu}, \\
S^{I}(0 \mid \mu, \nu) & =S_{222}^{I} \delta_{\mu \nu}+S_{221}^{I} p_{\mu} p_{\nu}, &
\end{aligned}
$$

with $I=A, C, D, E$, and we extract the scale dependence in complete analogy with Eq.(33).

Ultraviolet decompositions. The UV decomposition introduced in Eqs.(6) and (7) reads as

$$
s_{j}^{I}(s,\{m\})=\sum_{k=-2}^{0} s_{j}^{I}(s,\{m\} ; k) F_{k}^{2}(s),
$$

where $I=A, C, D, E$ classifies the family of integrals and $j$ contains the information about the tensor structure. It is worth noting that the following results can be written in a compact form through the one-loop, two-point functions introduced in Subsection 3.2. We shall be quoting the tensor configurations relevant for our calculation.

$\underline{\text { A family }}$

$$
\begin{gathered}
s_{0}^{A}\left(s,\{m\}_{123} ;-2\right)=2 \sum_{i=1}^{3} \frac{m_{i}^{2}}{s} \\
s_{0}^{A}\left(s,\{m\}_{123} ;-1\right)=\sum_{i=1}^{3} \frac{m_{i}^{2}}{s}\left[-2 \ln \left(\frac{m_{i}^{2}}{s}\right)+3\right]-\frac{1}{2} .
\end{gathered}
$$

$\underline{\text { C family }}$

$$
\begin{gathered}
s_{0}^{C}\left(s,\{m\}_{1234} ;-2\right)=-2, \quad s_{1}^{C}\left(s,\{m\}_{1234} ;-2\right)=\frac{1}{2}, \quad s_{2}^{C}\left(s,\{m\}_{1234} ;-2\right)=1, \\
s_{0}^{C}\left(s,\{m\}_{1234} ;-1\right)=-2 b_{0}\left(1,1, s,\{m\}_{34} ; 0\right)-1 \\
s_{1}^{C}\left(s,\{m\}_{1234} ;-1\right)=-b_{1}\left(1,1, s,\{m\}_{34} ; 0\right)+\frac{1}{8} \\
s_{2}^{C}\left(s,\{m\}_{1234} ;-1\right)=-2 b_{1}\left(1,1, s,\{m\}_{34} ; 0\right)+\frac{1}{4} .
\end{gathered}
$$

$\underline{D \text { family }}$

$$
\begin{aligned}
s_{0}^{D}\left(s,\{m\}_{12345} ;-2\right) & =0, \\
s_{112}^{D}\left(s,\{m\}_{12345} ;-2\right) & =-\frac{1}{2}, \quad s_{i}^{D}\left(s,\{m\}_{12345} ;-2\right)=0, \\
s_{i j 1}^{D}\left(s,\{m\}_{12345} ;-2\right) & =0,
\end{aligned}
$$




$$
\begin{array}{rlrl}
s_{0}^{D}\left(s,\{m\}_{12345} ;-1\right) & =0, & & s_{i}^{D}\left(s,\{m\}_{12345} ;-1\right)=0, \\
s_{112}^{D}\left(s,\{m\}_{12345} ;-1\right) & =-\frac{1}{2} b_{0}\left(1,1, s,\{m\}_{45} ; 0\right)-\frac{3}{8}, & & s_{122}^{D}\left(s,\{m\}_{12345} ;-1\right)=-\frac{1}{4}, \\
s_{222}^{D}\left(s,\{m\}_{12345} ;-1\right) & =-\frac{1}{2} b_{0}\left(1,1, s,\{m\}_{12} ; 0\right)-\frac{3}{8}, & \\
s_{i j 1}^{D}\left(s,\{m\}_{12345} ;-1\right) & =0 .
\end{array}
$$

$\underline{\text { E family }}$

$$
\begin{aligned}
& s_{0}^{E}\left(s,\{m\}_{12343} ;-2\right)=0, s_{i}^{E}\left(s,\{m\}_{12343} ;-2\right)=0, \\
& s_{112}^{E}\left(s,\{m\}_{12343} ;-2\right)=0, s_{122}^{E}\left(s,\{m\}_{12343} ;-2\right)=-\frac{1}{4}, \quad s_{222}^{E}\left(s,\{m\}_{12343} ;-2\right)=-\frac{1}{2}, \\
& s_{i j 1}^{E}\left(s,\{m\}_{12343} ;-2\right)=0, \\
& s_{0}^{E}\left(s,\{m\}_{12343} ;-1\right)=-2 b_{0}\left(2,1, s,\{m\}_{34} ; 0\right), \\
& s_{1}^{E}\left(s,\{m\}_{12343} ;-1\right)=-b_{1}\left(2,1, s,\{m\}_{34} ; 0\right), \\
& s_{2}^{E}\left(s,\{m\}_{12343} ;-1\right)=-2 b_{1}\left(2,1, s,\{m\}_{34} ; 0\right), \\
& s_{112}^{E}\left(s,\{m\}_{12343} ;-1\right)=\frac{1}{2} \frac{m_{1}^{2}+m m_{2}^{2}}{s} b_{0}\left(2,1, s,\{m\}_{34} ; 0\right)+\frac{1}{6} b_{21}\left(2,1, s,\{m\}_{34} ; 0\right), \\
& s_{111}^{E}\left(s,\{m\}_{12343} ;-1\right)=-\frac{2}{3} b_{21}\left(2,1, s,\{m\}_{34} ; 0\right), \\
& s_{122}^{E}\left(s,\{m\}_{12343} ;-1\right)=-b_{22}\left(2,1, s,\{m\}_{34} ; 0\right)-\frac{1}{16}, \\
& s_{121}^{E}\left(s,\{m\}_{12343} ;-1\right)=-b_{21}\left(2,1, s,\{m\}_{34} ; 0\right), \\
& s_{222}^{E}\left(s,\{m\}_{12343} ;-1\right)=-2 b_{22}\left(2,1, s,\{m\}_{34} ; 0\right)-\frac{1}{8}, \\
& s_{221}^{E}\left(s,\{m\}_{12343} ;-1\right)=-2 b_{21}\left(2,1, s,\{m\}_{34} ; 0\right) .
\end{aligned}
$$

The results shown for the $s^{E}$ family hold when $m_{3} \neq 0$.

Finite components. The UV-finite parts of two-point two-loop functions can be found in Ref. [10, Eq. (89) and Eqs. (146-147), for $S_{0}^{A}=S_{0}^{111}$; in Ref. [11], Subsection 5.8 for $S_{0}^{C}=S_{0}^{121}$, Subsection 7.3 for $S_{0}^{E}=S_{0}^{131}$ and Subsection 7.8 for $S_{0}^{D}=S_{0}^{221}$.

Special configurations. Some configurations where one or more masses vanish show overlapping UV and IR divergencies and deserve a separate discussion.

As a non trivial example, we consider the scalar function of the $C$ family evaluated at $p^{2}=0\left(s_{0}^{C}\right)$, since here the residue of the single UV pole can be IR divergent. Note that this function represents a tadpole, and it can be treated by means of the methods of Subsection 3.3. The residue of the double UV pole of $s_{0}^{C}$ can be found in Eq.(38) and the residue of the single UV pole is given in Eq.(39). The same UV components arise when $p^{2}=0$ and $m_{3}=m_{4}$ is not vanishing,

$$
s_{0}^{C}\left(0, m_{1}, m_{2}, m_{3}, m_{3}\right)=-2 F_{-2}^{2}\left(M^{2}\right)-F_{1}^{2}\left(M^{2}\right)\left(1-2 \ln \frac{m_{3}^{2}}{M^{2}}\right)+s_{\text {fin }}^{C},
$$

where $s_{\text {fin }}^{C}$ is finite when $\epsilon \rightarrow 0$. However, reduction in sub-loops requires to evaluate cases where $m_{3}=m_{4}=$ 0 . Here the residue of the single UV pole becomes IR singular, and the explicit expressions read as

$$
\begin{aligned}
s_{0}^{C}\left(0, m_{1}, m_{2}, 0,0\right) & =2 F_{-2}^{2}\left(M^{2}\right)+2\left[1+\frac{1}{m_{2}^{2}-m_{1}^{2}}\left(m_{1}^{2} \ln \frac{m_{1}^{2}}{M^{2}}-m_{2}^{2} \ln \frac{m_{2}^{2}}{M^{2}}\right)\right] F_{-1}^{2}\left(M^{2}\right)+F_{I R}, \\
F_{I R} & =-\frac{1}{\epsilon}\left(\frac{2}{\epsilon}-2 \Delta_{U V}+1\right) .
\end{aligned}
$$


Overlapping UV and IR divergencies appear also for special configurations of the scalar function of the $E$ family $\left(s_{0}^{E}\right)$, evaluated at arbitrary $s$, when $m_{3}=0$, i.e.

$$
\begin{aligned}
s_{0}^{E}\left(s, 0, m_{2}, 0, M, 0\right) & =\frac{2}{1-x}\left[\Delta_{U V}^{2}(s)-\frac{2}{\epsilon^{2}}\right]+2\left[\frac{1}{\epsilon}-\Delta_{U V}(s)\right]\left\{\frac { 1 } { 1 - x } \left[2 \Delta_{U V}(s)-1-\ln x\right.\right. \\
& \left.\left.+2 \ln (x-1)+\ln \frac{m_{2}^{2}}{s}\right]+\ln x-\ln (x-1)\right\}+s_{\text {fin }}^{E}\left(s, 0, m_{2}, 0, M, 0\right), \\
s_{0}^{E}\left(s, m_{1}, m_{2}, 0, M, 0\right) & =\frac{2}{1-x}\left[\Delta_{U V}^{2}(s)-\frac{2}{\epsilon^{2}}\right]+2\left[\frac{1}{\epsilon}-\Delta_{U V}(s)\right]\left\{\frac { 1 } { 1 - x } \left[2 \Delta_{U V}(s)-1\right.\right. \\
& \left.-\ln x+2 \ln (x-1)+\frac{m_{2}^{2}}{m_{2}^{2}-m_{1}^{2}} \ln \frac{m_{2}^{2}}{s}-\frac{m_{1}^{2}}{m_{2}^{2}-m_{1}^{2}} \ln \frac{m_{1}^{2}}{s}\right] \\
& +\ln x-\ln (x-1)\}+s_{\text {fin }}^{E}\left(s, m_{1}, m_{2}, 0, M, 0\right),
\end{aligned}
$$

where $s_{\text {fin }}^{E}$ is finite when $\epsilon \rightarrow 0$ and $x=M^{2} / s$. Note that here double poles arise, whereas the residue of the double UV pole vanishes in Eq.(42).

These spurious singularities are a consequence of the sub-loop reduction of Ref. [19. This technique allows to handle integrals with irreducible scalar products in the numerator and introduces new propagators with a corresponding zero mass. IR poles are then generated by the related integrals behaving as

$$
\int_{0} d^{n} q \frac{1}{q^{4}}
$$

The treatment of overlapping UV and IR poles is not free from ambiguities. Strictly speaking, the integrals of Eqs.(45) and (46) cannot be defined in any strip of the complex $n$-plane. UV regulation requires $n<4$ and IR one should be performed for $n>4$. Therefore, one should first of all disentangle overlapping UV and IR singularities. This can be achieved by means of the IBP identities introduced in Ref. [20], which allow to write the original integral as a combination of objects which are UV or IR singular but never both. At the end one can use the well-known relation between UV and IR regulators, i.e. $\hat{\epsilon}=-\epsilon$. We verified that this recipe gives the same result of the naive approach where one regularizes integrals for $n<4$ without separating the UV and the IR domains.

\subsection{Two-loop three-point integrals}

Notation. Our notation for three-point two-loop integrals can be found in Section 9 of Ref. 14. Here $P=p_{1}+p_{2}$ and we extract $s=-P^{2}$ in order to define scaled functions. For scalar configurations we introduce

$$
V_{0}^{E}=v_{0}^{E}, \quad V_{0}^{I, G}=s^{-1} v_{0}^{I, G}, \quad V_{0}^{M, K, H}=s^{-2} v_{0}^{M, K, H} .
$$

For tensor integrals, first we introduce form factors according to Section 9 of Ref. 14. Then, we define dimensionless functions in complete analogy with Eq.(48). The (somehow redundant) definition of the rooting of momenta in two-loop vertices has been introduced for all families in Ref. [14; we stick to the notation used there and one should only remember momentum conservation, i.e. $P=p_{1}+p_{2}$.

Ultraviolet decompositions. The UV decomposition introduced in Eqs.(6) and (17) reads as

$$
v_{j}^{L}(\{\text { momenta }\},\{m\})=\sum_{k=-2}^{0} v_{j}^{L}(\{\text { momenta }\},\{m\} ; k) F_{k}^{2}(s),
$$

where $L=E, I, G, M, K, H$ classifies the family of integrals under consideration and $j$ summarizes the information about the tensor structure. Here we show only UV-divergent tensor configurations relevant for our calculation.

$\underline{\text { E family }}$

$$
\begin{aligned}
& v_{0}^{E}(\cdots ;-2)=-2, \quad v_{11}^{E}(\cdots ;-2)=\frac{1}{2}, \quad v_{12}^{E}(\cdots ;-2)=1, \\
& v_{21}^{E}(\cdots ;-2)=1, \quad v_{22}^{E}(\cdots ;-2)=2 \text {, }
\end{aligned}
$$




$$
\begin{aligned}
& v_{0}^{E}\left(p_{2}, P,\{m\}_{1234} ;-1\right)=-2 b_{0}\left(1,1, p_{1},\{m\}_{34} ; 0\right)-1, \\
& v_{11}^{E}\left(p_{2}, P,\{m\}_{1234} ;-1\right)=-b_{1}\left(1,1, p_{1},\{m\}_{34} ; 0\right)+\frac{1}{8}, \\
& v_{12}^{E}\left(p_{2}, P,\{m\}_{1234} ;-1\right)=b_{0}\left(1,1, p_{1},\{m\}_{34} ; 0\right)+\frac{1}{4}, \\
& v_{21}^{E}\left(p_{2}, P,\{m\}_{1234} ;-1\right)=-2 b_{1}\left(1,1, p_{1},\{m\}_{34} ; 0\right)+\frac{1}{4}, \\
& v_{22}^{E}\left(p_{2}, P,\{m\}_{1234} ;-1\right)=2 b_{0}\left(1,1, p_{1},\{m\}_{34} ; 0\right)+\frac{1}{2} .
\end{aligned}
$$

\section{$\underline{\text { I family }}$}

$$
\begin{aligned}
& v_{0}^{I}(\cdots ;-2)=0, \quad v_{i j}^{I}(\cdots ;-2)=0, \\
& v_{114}^{I}(\cdots ;-2)=0, \quad v_{124}^{I}(\cdots ;-2)=-\frac{1}{4}, \quad v_{224}^{I}(\cdots ;-2)=-\frac{1}{2}, \\
& v_{i j k}^{I}(\cdots ;-2)=0, \\
& v_{0}^{I}\left(p_{1}, P,\{m\}_{12345} ;-1\right)=-2 c_{0}\left(1,1,1, p_{1}, p_{2}, P,\{m\}_{345} ; 0\right), \\
& v_{1 i}^{I}\left(p_{1}, P,\{m\}_{12345} ;-1\right)=-c_{1 i}\left(1,1,1, p_{1}, p_{2}, P,\{m\}_{345} ; 0\right), \\
& v_{2 i}^{I}\left(p_{1}, P,\{m\}_{12345} ;-1\right)=-2 c_{1 i}\left(1,1,1, p_{1}, p_{2}, P,\{m\}_{345} ; 0\right), \\
& v_{114}^{I}\left(p_{1}, P,\{m\}_{12345} ;-1\right)=\frac{1}{2} \frac{m_{1}^{2}+m_{2}^{2}}{s} c_{0}\left(1,1,1, p_{1}, P,\{m\}_{345} ; 0\right) \\
&+\frac{1}{6} \frac{p_{1}^{2}}{s} c_{21}\left(1,1,1, p_{1}, p_{2}, P,\{m\}_{345} ; 0\right)+\frac{1}{6} \frac{P^{2}-p_{1}^{2}-p_{2}^{2}}{s} c_{23}\left(1,1,1, p_{1}, p_{2}, P,\{m\}_{345} ; 0\right) \\
& \quad \frac{1}{s} c_{22}\left(1,1,1, p_{1}, p_{2}, P,\{m\}_{345} ; 0\right), \\
& v_{11 i}^{I}\left(p_{1}, P,\{m\}_{12345} ;-1\right)=-\frac{2}{3} c_{2 i}\left(1,1,1, p_{1}, p_{2}, P,\{m\}_{345} ; 0\right), \\
& v_{124}^{I}\left(p_{1}, P,\{m\}_{12345} ;-1\right)=-c_{24}\left(1,1,1, p_{1}, p_{2}, P,\{m\}_{345} ; 0\right)-\frac{1}{16}, \\
& v_{12 i}^{I}\left(p_{1}, P,\{m\}_{12345} ;-1\right)=-c_{2 i}\left(1,1,1, p_{1}, p_{2}, P,\{m\}_{345} ; 0\right), \\
& v_{224}^{I}\left(p_{1}, P,\{m\}_{12345} ;-1\right)=-2 c_{24}\left(1,1,1, p_{1}, p_{2}, P,\{m\}_{345} ; 0\right)-\frac{1}{8}, \\
& v_{22 i}^{I}\left(p_{1}, P,\{m\}_{12345} ;-1\right)=-2 c_{2 i}\left(1,1,1, p_{1}, p_{2}, P,\{m\}_{345} ; 0\right) .
\end{aligned}
$$

G family

$$
\begin{aligned}
v_{0}^{G}(\cdots ;-2) & =0, \quad v_{i j}^{G}(\cdots ;-2)=0, \\
v_{114}^{G}(\cdots ;-2) & =-\frac{1}{2}, \quad v_{124}^{G}(\cdots ;-2)=0, \quad v_{224}^{G}(\cdots ;-2)=-\frac{1}{2}, \\
v_{i j k}^{G}(\cdots ;-2) & =0,
\end{aligned}
$$

$$
\begin{aligned}
v_{0}^{G}(\cdots ;-1) & =0, \quad v_{1 i}^{G}(\cdots ;-1)=0, \\
v_{114}^{G}\left(p_{1}, p_{1}, P,\{m\}_{12345} ;-1\right) & =-\frac{1}{2} b_{0}\left(1,1, p_{2},\{m\}_{45} ; 0\right)-\frac{3}{8},
\end{aligned}
$$$$
v_{124}^{G}\left(p_{1}, p_{1}, P,\{m\}_{12345} ;-1\right)=-\frac{1}{4} \text {, }
$$$$
v_{224}^{G}\left(p_{1}, p_{1}, P,\{m\}_{12345} ;-1\right)=-\frac{1}{2} b_{0}\left(1,1, p_{1},\{m\}_{12} ; 0\right)-\frac{3}{8} \text {. }
$$ 
$\underline{M \text { family }}$

$$
\begin{aligned}
v_{0}^{M}\left(p_{1}, P,\{m\}_{123453} ;-1\right) & =-2 c_{0}\left(2,1,1, p_{1}, p_{2}, P,\{m\}_{345} ; 0\right), \\
v_{1 i}^{M}\left(p_{1}, P,\{m\}_{123453} ;-1\right) & =-c_{1 i}\left(2,1,1, p_{1}, p_{2}, P,\{m\}_{345} ; 0\right), \\
v_{2 i}^{M}\left(p_{1}, P,\{m\}_{123453} ;-1\right) & =-2 c_{1 i}\left(2,1,1, p_{1}, p_{2}, P,\{m\}_{345} ; 0\right), \\
v_{114}^{M}\left(p_{1}, P,\{m\}_{123453} ;-1\right) & =\frac{1}{2} \frac{m_{1}^{2}+m_{2}^{2}}{s} c_{0}\left(2,1,1, p_{1}, p_{2}, P,\{m\}_{345} ; 0\right) \\
+\frac{1}{6} \frac{p_{1}^{2}}{s} c_{21}\left(2,1,1, p_{1}, p_{2}, P,\{m\}_{345} ; 0\right) & +\frac{1}{6} \frac{P^{2}-p_{1}^{2}-p_{2}^{2}}{s} c_{23}\left(2,1,1, p_{1}, p_{2}, P,\{m\}_{345} ; 0\right) \\
& +\frac{1}{6} \frac{p_{2}^{2}}{s} c_{22}\left(2,1,1, p_{1}, p_{2}, P,\{m\}_{345} ; 0\right), \\
v_{11 i}^{M}\left(p_{1}, P,\{m\}_{123453} ;-1\right) & =-\frac{2}{3} c_{2 i}\left(2,1,1, p_{1}, p_{2}, P,\{m\}_{345} ; 0\right), \\
v_{12 i}^{M}\left(p_{1}, P,\{m\}_{123453} ;-1\right) & =-c_{2 i}\left(2,1,1, p_{1}, p_{2}, P,\{m\}_{345} ; 0\right), \\
v_{22 i}^{M}\left(p_{1}, P,\{m\}_{123453} ;-1\right) & =-2 c_{2 i}\left(2,1,1, p_{1}, p_{2}, P,\{m\}_{345} ; 0\right) .
\end{aligned}
$$

$\underline{H, K \text { families }}$

$$
\begin{aligned}
v_{0}^{K}(\cdots ;-1) & =0, \quad v_{1 i}^{K}(\cdots ;-1)=0, \\
v_{114}^{K}\left(P, p_{1}, P,\{m\}_{123456} ;-1\right) & =-\frac{1}{2} c_{0}\left(1,1,1, p_{1}, p_{2}, P,\{m\}_{456} ; 0\right), \\
v_{i j k}^{K}(\cdots ;-1) & =0, \quad v_{i j k}^{H}(\cdots ;-1)=0 .
\end{aligned}
$$

\section{$4 \overline{M S}$ renormalization at two loops}

We now briefly summarize the notion of renormalization procedure. Any renormalization procedure for a gauge theory requires three steps:

- the definition of the gauge-fixing term of the Lagrangian;

- the renormalization-scheme prescription;

- the choice of an IPS.

In Subsection 4.1 we define the gauge-fixing part of the Standard-Model Lagrangian and in Subsection 4.2 we discuss the renormalization scheme employed in this paper. This is a rather technical subject, but one should realize that choosing a renormalization scheme is equivalent to changing the variables which describe the degrees of freedom of the theory. Bare quantities are traded for renormalized ones through multiplicative renormalization constants, and the latter are expanded in perturbation theory introducing counterterms. The prescription imposed for the counterterms defines the so-called renormalization scheme.

Whatever the renormalization scheme employed, a theory becomes predictive when renormalized quantities are expressed in terms of the chosen IPS. As long as the same data are employed, different renormalization schemes lead to the same theoretical predictions. Special care should be devoted to the choice of the POs used as input: two predictions for the same PO differ by an amount proportional to the missing higher-order corrections if they refer to different IPS. We will discuss this issue in details in III, where we will show that a consistent description of unstable particles at two loops can be achieved in the framework of complex poles [21].

\subsection{Gauge fixing}

We fix the gauge for the electroweak sector of the Standard-Model Lagrangian introducing six gauge parameters $\xi_{i}\left(i=A, Z, A Z, \varphi^{0}, W, \varphi\right)$,

$$
\mathcal{L}_{g f}^{E W}=-\mathcal{C}^{+} \mathcal{C}^{-}-\frac{1}{2}\left[\left(\mathcal{C}^{A}\right)^{2}+\left(\mathcal{C}^{Z}\right)^{2}\right]
$$




$$
\mathcal{C}^{A}=-\frac{1}{\xi_{A}} \partial_{\mu} A_{\mu}-\xi_{A Z} \partial_{\mu} Z_{\mu}, \quad \mathcal{C}^{Z}=-\frac{1}{\xi_{Z}} \partial_{\mu} Z_{\mu}+\xi_{\varphi^{0}} M_{0} \varphi^{0}, \quad \mathcal{C}^{ \pm}=-\frac{1}{\xi_{W}} \partial_{\mu} W_{\mu}^{ \pm}+\xi_{\varphi} M \varphi^{ \pm} .
$$

Here $A_{\mu}, Z_{\mu}$ and $W_{\mu}^{ \pm}$are the fields for the photon and the $Z$ and the $W$ bosons and $\varphi^{0}$ and $\varphi^{ \pm}$are the fields for the neutral and charged unphysical Higgs-Kibble scalars. For the QCD sector, we employ the usual 't Hooft-Feynman gauge.

Note that gauge parameters are usually introduced in order to test predictions for physical (pseudo)observables (hereafter POs); $S$-matrix elements are gauge independent, and one can indeed check that gauge-parameter dependence cancels out. Here, instead, the gauge-fixing term which defines what we call the $R_{\xi \xi}$ gauge is closely related to our renormalization strategy. We will discuss this issue in Subsection 4.2 . where we will introduce renormalized gauge parameters.

Dyson-resummed propagators in the $R_{\xi \xi}$ gauge. In this Section we illustrate the method of Dyson resummation of higher-order corrections which plays a crucial role in any renormalization procedure, because mass renormalization and wave-function factors for external legs naturally arise in this context. In the $R_{\xi \xi}$ gauge, in addition, transitions between the vector gauge bosons and the Higgs-Kibble scalars, as well as a mixing between the photon and the $Z$ boson, take place at the Born level. Therefore, Dyson resummation is a mandatory step already at lowest order in perturbation theory.

Here we show the Dyson-resummed propagators for the vector gauge bosons and the Higgs-Kibble scalars, including all the allowed transitions. Furthermore, we provide the explicit expressions for the propagators of the Faddeev-Popov ghost fields.

The charged sector: The Dyson-resummed propagator for the $W$ boson reads as

$$
\bar{\Delta}_{\mu \nu}^{W W}=\frac{1}{(2 \pi)^{4} i} \frac{1}{p^{2}+M^{2}}\left(\delta_{\mu \nu}+\bar{\Delta}_{p p}^{W W} p_{\mu} p_{\nu}\right)
$$

where we introduced

$$
\bar{\Delta}_{p p}^{W W}=\frac{1}{p^{2}+\xi_{W}^{2} M^{2}}\left[\xi_{W}^{2}-1+\frac{\left(\xi_{W}-\xi_{\varphi}\right)^{2} \xi_{W}^{2} M^{2}\left(p^{2}+M^{2}\right)}{\left(p^{2}+\xi_{W} \xi_{\varphi} M^{2}\right)^{2}}\right] .
$$

Moreover, for the propagator of the $\varphi$ boson we get

$$
\bar{\Delta}^{\varphi \varphi}=\frac{1}{(2 \pi)^{4} i} \frac{p^{2}+\xi_{W}^{2} M^{2}}{\left(p^{2}+\xi_{W} \xi_{\varphi} M^{2}\right)^{2}} .
$$

Finally, a $W-\varphi$ transition takes place,

$$
\bar{\Delta}_{\mu}^{W \varphi}=\frac{1}{(2 \pi)^{4} i} \frac{M \xi_{W}\left(\xi_{\varphi}-\xi_{W}\right)}{\left(p^{2}+\xi_{W} \xi_{\varphi} M^{2}\right)^{2}} i p_{\mu} .
$$

The neutral sector: Let us start with the Dyson-resummed propagators for the photon and the $Z$ boson,

$$
\bar{\Delta}_{\mu \nu}^{A A}=\frac{1}{(2 \pi)^{4} i} \frac{1}{p^{2}}\left(\delta_{\mu \nu}+\bar{\Delta}_{p p}^{A A} p_{\mu} p_{\nu}\right), \quad \bar{\Delta}_{\mu \nu}^{Z Z}=\frac{1}{(2 \pi)^{4} i} \frac{1}{p^{2}+M_{0}^{2}}\left(\delta_{\mu \nu}+\bar{\Delta}_{p p}^{Z Z} p_{\mu} p_{\nu}\right),
$$

where we defined

$$
\begin{gathered}
\bar{\Delta}_{p p}^{A A}=\frac{\xi_{A}^{2}-1}{p^{2}}+\frac{\xi_{A}^{2} \xi_{A Z}^{2} \xi_{Z}^{2}\left(p^{2}+\xi_{\varphi^{0}}^{2} M_{0}^{2}\right)}{\left(p^{2}+\xi_{z} \xi_{\varphi^{0}} M_{0}^{2}\right)^{2}} \\
\bar{\Delta}_{p p}^{Z Z}=\frac{1}{p^{2}+\xi_{C}^{2} M_{0}^{2}}\left[\xi_{C}^{2}-1+\xi_{C}^{2}\left(p^{2}+M_{0}^{2}\right) \frac{M_{0}^{2}\left(\xi_{z}-\xi_{\varphi^{0}}\right)^{2}+\xi_{A Z}^{2} \xi_{Z}^{2}\left(p^{2}+\xi_{\varphi^{0}}^{2} M_{0}^{2}\right)}{\left(p^{2}+\xi_{\varphi^{0}} \xi_{Z} M_{0}^{2}\right)^{2}}\right],
\end{gathered}
$$

and we introduced a combination of gauge parameters, $\xi_{C}^{2}=\xi_{z}^{2} /\left(1+\xi_{z}^{2} \xi_{A Z}^{2}\right)$. Furthermore, the propagator for the $\varphi^{0}$ boson reads as

$$
\bar{\Delta}^{\varphi^{0} \varphi^{0}}=\frac{1}{(2 \pi)^{4} i} \frac{p^{2}+\xi_{z}^{2} M_{0}^{2}}{\left(p^{2}+\xi_{z} \xi_{\varphi^{0}} M_{0}^{2}\right)^{2}} .
$$


In the neutral sector we have vector-scalar transitions,

$$
\bar{\Delta}_{\mu}^{A \varphi^{0}}=\frac{1}{(2 \pi)^{4} i} \frac{M_{0} \xi_{z} \xi_{A} \xi_{A Z}\left(\xi_{\varphi^{0}}-\xi_{z}\right)}{\left(p^{2}+\xi_{Z} \xi_{\varphi^{0}} M_{0}^{2}\right)^{2}} i p_{\mu}, \quad \bar{\Delta}_{\mu}^{Z \varphi^{0}}=\frac{1}{(2 \pi)^{4} i} \frac{M_{0} \xi_{Z}\left(\xi_{\varphi^{0}}-\xi_{z}\right)}{\left(p^{2}+\xi_{Z} \xi_{\varphi^{0}} M_{0}^{2}\right)^{2}} i p_{\mu},
$$

as well as a mixing between the photon and the $Z$ boson,

$$
\bar{\Delta}_{\mu \nu}^{A Z}=\frac{1}{(2 \pi)^{4} i} \frac{\xi_{A} \xi_{A Z} \xi_{z}^{2}\left(p^{2}+\xi_{\varphi^{0}}^{2} M_{0}^{2}\right)}{p^{2}\left(p^{2}+\xi_{Z} \xi_{\varphi^{0}} M_{0}^{2}\right)^{2}} p_{\mu} p_{\nu} .
$$

Ghost fields: Finally, the gauge-fixing Lagrangian defines the propagators for the charged and neutral ghost fields $X^{ \pm}, Y^{z}$ and $Y^{A}$,

$$
\Delta^{X^{ \pm}}=\frac{1}{(2 \pi)^{4} i} \frac{\xi_{W}}{p^{2}+\xi_{\varphi} \xi_{W} M^{2}}, \quad \Delta^{Y^{Z}}=\frac{1}{(2 \pi)^{4} i} \frac{\xi_{z}}{p^{2}+\xi_{\varphi^{0}} \xi_{Z} M_{0}^{2}}, \quad \Delta^{Y^{A}}=\frac{1}{(2 \pi)^{4} i} \frac{\xi_{A}}{p^{2}} .
$$

\subsection{Definition of the renormalization scheme: $\overline{M S}$ and beyond}

The orthodox approach to renormalization [6] uses the language of counterterms. It is worth noting that this is not a mandatory step, since one could write directly renormalization equations that connect the bare parameters of the Lagrangian to an IPS, skipping the introduction of intermediate renormalized quantities and avoiding any unnecessary reference to a given renormalization scheme. In this approach, carried on at one loop in [22, no special attention is paid to individual Green functions, and one is mainly concerned with:

- UV finiteness of $S$-matrix elements after the proper treatment of external legs in amputated Green functions, which greatly reduces the complexity of the calculation;

- gauge independence of predictions for (pseudo-)observables.

However, renormalization equations are usually organized through different building blocks, where gaugeboson self-energies embed process-independent (universal) higher-order corrections and play a privileged role. Therefore, their structure has to be carefully analyzed, and the language of counterterms allows to disentangle UV overlapping divergencies which show up at two loops. In a renormalizable gauge theory, in fact, the UV poles of any Green function can be removed order-by-order in perturbation theory. In addition, the imaginary part of a Green function at a given order is fixed, through unitarity constraints, by the previous orders. Therefore, UV-subtraction terms have to be at most polynomials in the external momenta (in the following, local subtraction terms). In this paper, we will express our results using the language of counterterms:

- we promote bare quantities (parameters and fields) to renormalized ones;

- we fix the counterterms at one loop in order to remove the UV poles from all one-loop Green functions;

- at this stage, we check that two-loop Green functions develop local UV residues;

- our final task is to fix the counterterms at two loops and to remove the UV poles from two-loop Green functions.

Obviously, the absorption of UV divergencies into local counterterms does not exhaust the renormalization procedure, because we have still to connect renormalized quantities to POs, thus making the theory predictive. This will be the subject of III. In the remainder of this section we discuss renormalization constants for all parameters and fields.

\section{Renormalization constants}

We relate bare quantities to renormalized ones introducing multiplicative renormalization constants $Z_{i}$ and (if not otherwise stated) we expand them through the renormalized $S U(2)$ coupling constant $g_{R}$,

$$
Z_{i}=1+\sum_{n=1}^{\infty}\left(\frac{g_{R}^{2}}{16 \pi^{2}}\right)^{n} \delta Z_{i}^{(n)}
$$


where $\delta Z_{i}^{(n)}$ are counterterms and the subscript $i$ refers to masses, couplings, gauge parameters and fields. Let us make more precise the notion of renormalization constants.

- for the bare masses of the $W$ boson $(M)$, the Higgs boson $\left(M_{H}\right)$ and the fermions $\left(m_{f}\right)$ we write $m=Z_{m}^{1 / 2} m_{R}$, where $m=M, M_{H}, m_{f}$ and $m_{R}$ corresponds to one of the associated renormalized masses. $Z_{m}$ is expanded through Eq.(69).

- For the bare $S U(2)$ coupling constant $g$ and the bare cosine (sine) of the weak-mixing angle $c_{\theta}\left(s_{\theta}\right)$ we define $p=Z_{p} p_{R}$, where $p=g, c_{\theta}, s_{\theta}$ and $p_{R}$ is one of the related renormalized parameters. $Z_{p}$ is expanded through Eq. (69).

- For the bare gauge parameters introduced in Eq.(58) we use $\xi=Z_{\xi} \xi_{R}$, where $\xi$ is one of the bare gauge parameters and $\xi_{R}$ is the associated renormalized quantity. $Z_{\xi}$ is expanded by means of Eq.(69) except for the case $\xi=\xi_{A Z}$, where we use

$$
Z_{\xi_{A Z}}=\sum_{n=1}^{\infty}\left(\frac{g_{R}^{2}}{16 \pi^{2}}\right)^{n} \delta Z_{\xi_{A Z}}^{(n)}
$$

After the expansions, we use the freedom in choosing the values for the renormalized gauge parameters and we set $\xi=1$, thus compromising between two alternative exigencies. On the one hand, counterterms for the gauge parameters are required to remove UV divergencies; on the other hand, we recover part of the simplicity of 't Hooft-Feynman gauge.

- For a given bare field $\phi$ it is convenient to write $\phi=Z_{\phi}^{1 / 2} \phi_{R}$, where $\phi_{R}$ is a renormalized field, and we expand $Z_{\phi}$ through Eq.(69). The bare photon field $A^{\mu}$ represents an exception, and here we use

$$
A^{\mu}=Z_{A A}^{1 / 2} A_{R}^{\mu}+Z_{A Z}^{1 / 2} Z_{R}^{\mu}, \quad Z_{A Z}^{1 / 2}=\sum_{n=1}^{\infty}\left(\frac{g_{R}^{2}}{16 \pi^{2}}\right)^{n} \delta Z_{A Z}^{(n)},
$$

where $A_{R}^{\mu}$ and $Z_{R}^{\mu}$ are the renormalized fields for the photon and the $Z$ boson. Note that $Z_{A A}$ is expanded through Eq.(69).

In addition, bare fermion fields $\psi$ (we omit flavour labels) are written by means of bare left-handed and right-handed chiral fields $\psi^{L}$ and $\psi^{R}$. The latter are traded for renormalized fields $\psi_{R}^{L}$ and $\psi_{R}^{R}$ expanding the renormalization constants through Eq.(69),

$$
\psi^{L, R}=\frac{1}{2}\left(1 \pm \gamma^{5}\right) \psi, \quad \psi^{L, R}=Z_{\psi_{L, R}}^{1 / 2} \psi_{R}^{L, R} .
$$

Finally, Faddeev-Popov ghost fields are not renormalized.

\section{UV decompositions for Green functions}

Before defining our renormalization scheme, we introduce UV decompositions also for Green functions. Given a one- $(i=1)$ or two-loop $(i=2)$ Green function with $N$ external lines carrying Lorentz indices $\mu_{j}$, $j=1, \ldots, N$, we introduce form factors,

$$
G_{\mu_{1} \ldots \mu_{N}}^{i}=\sum_{a=1}^{A} G_{a}^{i} K_{\mu_{1} \ldots \mu_{N}}^{a}, \quad i=1,2 .
$$

Here the set $K^{a}$, with $a=1, \ldots, A$, contains independent tensor structures made up of external momenta, Kronecker-delta functions, elements of the Clifford algebra and Levi-Civita tensors. Next, we introduce UV decompositions for the form factors $G_{a}^{i}$ in analogy with Eqs.(4)-(7),

$$
1 \text { loop } \rightarrow G_{a}^{1}=\sum_{k=-1}^{1} G_{a ; k}^{1} F_{k}^{1}\left(M_{R}^{2}\right), \quad 2 \text { loops } \rightarrow G_{a}^{2}=\sum_{k=-2}^{0} G_{a ; k}^{2} F_{k}^{2}\left(M_{R}^{2}\right) \text {. }
$$


The UV factors $F_{k}^{1}(k=-1,0,1)$ and $F_{k}^{2}(k=-2,-1,0)$ can be read in Eqs.(15) and (77).

\section{Renormalization prescriptions: $\overline{M S}$ and beyond}

In the spirit of the UV decomposition of Eq. (174), one could define a non-minimal $(\overline{N M S})$ subtraction scheme where the one- and two-loop counterterms are defined by

$$
1 \text { loop } \rightarrow \delta Z_{i}^{(1)}=\Delta Z_{i}^{(1)} F_{-1}^{1}\left(M_{R}^{2}\right), \quad 2 \text { loops } \rightarrow \delta Z_{i}^{(2)}=\sum_{k=-2}^{-1} \Delta Z_{i ; k}^{(2)} F_{k}^{2}\left(M_{R}^{2}\right),
$$

and are fixed in order to remove order-by-order the poles at $\epsilon=0$ for any Green function. As a result, also the product of a one-loop counterterm with a one-loop diagram (i.e., a one-loop counterterm insertion) has the same UV decomposition of a two-loop function thus simplifying two-loop renormalized Green functions. Even if we scale non-tadpole diagrams with $s$ instead of $M_{R}^{2}$, the difference is proportional to $\ln M_{R}^{2} /(s \epsilon)$, a non-local term which cancels in the total.

Although the $\overline{N M S}$ scheme has the virtue of respecting a universal UV decomposition, we adopt the conventional $\overline{M S}$ choice, keeping the counterterms as simple as possible. Let us accordingly define

$$
\delta Z_{i}^{(1)}=\left(-\frac{2}{\epsilon}+C\right) \Delta Z_{i}^{(1)}
$$

and we define the renormalization scheme choosing the explicit value for $C$. We define a minimal $\overline{M S}$ subtraction scheme,

$$
\delta Z_{i}^{(1)}=\left(-\frac{2}{\epsilon}+\Delta_{U V}\right) \Delta Z_{i}^{(1)}
$$

and we fix the counterterms in order to remove the poles at $\epsilon=0$ for any one-loop Green function. Next, we extend the scheme at two loops by introducing

$$
\delta Z_{i}^{(2)}=\left(\frac{1}{\epsilon}-\Delta_{U V}\right)\left(\frac{\Delta Z_{i ; 1}^{(2)}}{\epsilon}+\Delta Z_{i ; 2}^{(2)}\right)+\Delta_{U V}^{2} \Delta Z_{i ; 3}^{(2)} .
$$

Accordingly, we decompose the two-loop form factors,

$$
G_{a}^{2}=\left(\frac{1}{\epsilon^{2}}-\frac{\Delta_{U V}}{\epsilon}\right) G_{a ; U V ; 1}^{2}+\left(\frac{1}{\epsilon}-\Delta_{U V}\right) G_{a ; U V ; 2}^{2}+\Delta_{U V}^{2} G_{a ; U V ; 3}^{2}+G_{a ; F}^{2},
$$

and we fix the counterterms in order to remove the poles at $\epsilon=0$ for any two-loop Green function. Note the correspondence with the decomposition in Eq.(74),

$$
\begin{array}{ll}
G_{a ; U V ; 1}^{2}=G_{a ;-2}^{2}, & G_{a ; U V ; 2}^{2}=G_{a ;-1}^{2}, \\
G_{a ; U V ; 3}^{2}=\frac{1}{2} G_{a ;-2}^{2}, & G_{a ; F}^{2}=G_{a ; 0}^{2} .
\end{array}
$$

\section{One-loop renormalization}

In this section we collect all the one-loop results necessary for our renormalization procedure. Tadpole renormalization ( $\beta$ vertices) and neutral-sector diagonalization ( $\Gamma$ vertices) are shown in Subsection [5.1] and counterterms for parameters and fields are presented in Subsection 5.2 .

In Subsection 5.3 we discuss the relevance of one-loop finite renormalization through some examples and in Subsection 5.4 we analyze how counterterms affect WST identities. Renormalized WST identities riafferm the basic simplicity of the one-loop structure of the theory. These results should provide a useful guide to the two-loop renormalization. 


\section{$5.1 \beta$ and $\Gamma$ at one loop}

Two key ingredients are the parameter $\beta_{t}$, introduced in Sect. 2.3 of I (tadpole renormalization), and the parameter $\Gamma$, defined in Sect. 3.1 of I (all-order diagonalization of the neutral sector). We recall that one can define different tadpole schemes, and in I, we discussed the so-called $\beta_{h}$ and $\beta_{t}$ schemes; since here no ambiguities arise, we drop everywhere the subscript $t$ from $\beta$. To show the one-loop results, we expand $\beta$ and $\Gamma$ in the unrenormalized $S U(2)$ coupling constant $g$,

$$
\beta=\sum_{n=1}^{\infty} g^{2 n} \beta_{n}, \quad \Gamma=\sum_{n=1}^{\infty} g^{2 n} \Gamma_{n} .
$$

The complete answer for $\beta_{1}$ reads as

$$
\begin{aligned}
\beta_{1} & =\frac{1}{16 \pi^{2} x_{H}}\left\{3 \sum_{i=1}^{3}\left[x_{d, i}^{2} a_{0}\left(m_{d, i}\right)+x_{u, i}^{2} a_{0}\left(m_{u, i}\right)\right]+\sum_{i=1}^{3} x_{l, i}^{2} a_{0}\left(m_{l, i}\right)\right. \\
& \left.-\frac{1}{4 c_{\theta}^{2}}\left(\frac{n-1}{c_{\theta}^{2}}+\frac{x_{H}}{2}\right) a_{0}\left(M_{0}\right)-\frac{1}{2}\left(n-1+\frac{x_{H}}{2}\right) a_{0}(M)-\frac{3}{8} x_{H}^{2} a_{0}\left(M_{H}\right)\right\},
\end{aligned}
$$

where we recall that $x_{i}=m_{i}^{2} / M^{2}, l_{i}$ is the charged lepton and $u_{i}, d_{i}$ are the up and down quarks of the $i$ th fermion doublet. The one-loop tadpole $a_{0}$ is given in Eq.(13) and the explicit result for $\Gamma_{1}$ is

$$
\Gamma_{1}=\frac{n-2}{16 \pi^{2}} a_{0}(M)
$$

Note that in Eqs.(82) and (83) we dropped the subscript $R$, since bare quantities disappear after the introduction of renormalized parameters and fields.

\subsection{One-loop counterterms}

In this subsection we provide the full list of one-loop counterterms in the $\overline{M S}$ scheme. Counterterms are defined in Eqs.(69)-(72), and UV factors are extracted through Eq.(77). Here we introduce short-hand notations for sums over fermions ( $l \rightarrow$ charged leptons, $u, d \rightarrow$ quarks),

$$
X_{l}^{j}=\sum_{i=1}^{3} x_{l, i}^{j}, \quad X_{u}^{j}=\sum_{i=1}^{3} x_{u, i}^{j}, \quad X_{d}^{j}=\sum_{i=1}^{3} x_{d, i}^{j} .
$$

Gauge parameters. Gauge-parameter counterterms can be expressed by means of field, mass and couplingconstant counterterms,

$$
\begin{array}{ll}
\Delta Z_{\xi_{A}}^{(1)}=\frac{1}{2} \Delta Z_{A A}^{(1)}, & \Delta Z_{\xi_{A Z}}^{(1)}=-\Delta Z_{A Z}^{(1)}, \\
\Delta Z_{\xi_{Z}}^{(1)}=\frac{1}{2} \Delta Z_{Z}^{(1)}, & \Delta Z_{\xi_{\varphi^{0}}}^{(1)}=-\frac{1}{2}\left(\Delta Z_{\varphi^{0}}^{(1)}+\Delta Z_{M}^{(1)}\right)+\Delta Z_{c_{\theta}}^{(1)}, \\
\Delta Z_{\xi_{W}}^{(1)}=\frac{1}{2} \Delta Z_{W}^{(1)}, & \Delta Z_{\xi_{\varphi}}^{(1)}=-\frac{1}{2}\left(\Delta Z_{\varphi}^{(1)}+\Delta Z_{M}^{(1)}\right) .
\end{array}
$$

The gauge-fixing term defined in Eq.(58) is invariant under renormalization. We will discuss this issue in Subsection 5.4.

Gauge-boson and Higghs-Kibble fields.

$$
\begin{aligned}
\Delta Z_{A A}^{(1)} & =\frac{23}{3} s_{\theta}^{2}, & \Delta Z_{A Z}^{(1)} & =-\frac{s_{\theta}}{3}\left(\frac{41}{2} \frac{1}{c_{\theta}}-23 c_{\theta}\right), \\
\Delta Z_{Z}^{(1)} & =\frac{1}{3}\left(\frac{41}{2} \frac{1}{c_{\theta}^{2}}-41+23 c_{\theta}^{2}\right), & \Delta Z_{\varphi^{0}}^{(1)} & =-1-\frac{1}{2}\left[\frac{1}{c_{\theta}^{2}}-X_{l}-3\left(X_{d}+X_{u}\right)\right], \\
\Delta Z_{W}^{(1)} & =\frac{5}{6}, & \Delta Z_{\varphi}^{(1)} & =\Delta Z_{\varphi^{0}}^{(1)} .
\end{aligned}
$$


Masses and couplings. Here we discover a nice feature of the neutral-sector diagonalization introduced in Sect. 3.1 of I, since in the full-fledged Standard Model we derive a QED-like relation involving $\Delta Z_{g}^{(1)}, \Delta Z_{s_{\theta}}^{(1)}$ and $\Delta Z_{A A}^{(1)}$,

$$
\begin{aligned}
& \Delta Z_{M}^{(1)}=-\frac{3}{4} \frac{1}{c_{\theta}^{2}}-\frac{7}{3}+\frac{1}{x_{H}}\left[\frac{3}{2} \frac{1}{c_{\theta}^{4}}+3-2 X_{l}^{2}-6\left(X_{d}^{2}+X_{u}^{2}\right)\right]+\frac{1}{2}\left[\frac{3}{2} x_{H}+X_{l}+3\left(X_{d}+X_{u}\right)\right], \\
& \Delta Z_{c_{\theta}}^{(1)}=\frac{1}{2}\left(\frac{41}{6} \frac{1}{c_{\theta}^{2}}-\frac{29}{2}+\frac{23}{3} c_{\theta}^{2}\right), \quad \Delta Z_{s_{\theta}}^{(1)}=-\frac{c_{\theta}^{2}}{s_{\theta}^{2}} \Delta Z_{c_{\theta}}^{(1)}, \quad \Delta Z_{g}^{(1)}=-\Delta Z_{s_{\theta}}^{(1)}-\frac{1}{2} \Delta Z_{A A}^{(1)} .
\end{aligned}
$$

In other words, electric-charge renormalization depends only on the photon vacuum-polarization function, also in the (complete) Standard Model.

\section{Higgs-boson field and mass.}

$$
\Delta Z_{H}^{(1)}=-1-\frac{1}{2}\left[\frac{1}{c_{\theta}^{2}}-X_{l}-3\left(X_{d}+X_{u}\right)\right], \quad \Delta Z_{M_{H}}^{(1)}=\frac{3}{2}\left[\frac{1}{2} \frac{1}{c_{\theta}^{2}}+1-\frac{1}{2} x_{H}-\frac{1}{3} X_{l}-\left(X_{d}+X_{u}\right)\right] .
$$

Fermion fields and masses. Here $\nu$ is the neutrino field associated with the charged lepton $l$, and $u$ and $d$ are up and down quarks belonging to the same doublet. Furthermore, $g_{s}$ is the $S U(3)$ coupling constant of strong interactions. For left-handed spinor-field counterterms we get

$$
\begin{aligned}
\Delta Z_{\nu_{L}}^{(1)} & =\frac{1}{2}\left(\frac{1}{2} \frac{1}{c_{\theta}^{2}}+1+\frac{1}{2} x_{l}\right), & \Delta Z_{u_{L}}^{(1)} & =\frac{1}{4}\left(\frac{1}{9} \frac{1}{c_{\theta}^{2}}+\frac{26}{9}+x_{u}+x_{d}+\frac{16}{3} \frac{g_{s}^{2}}{g^{2}}\right), \\
\Delta Z_{l_{L}}^{(1)} & =\Delta Z_{\nu_{L}}^{(1)}, & \Delta Z_{d_{L}}^{(1)} & =\Delta Z_{u_{L}}^{(1)} .
\end{aligned}
$$

Expressions for the right-handed components are given by

$$
\begin{aligned}
\Delta Z_{\nu_{R}}^{(1)} & =0, & \Delta Z_{u_{R}}^{(1)} & =\frac{4}{9} \frac{1}{c_{\theta}^{2}}-\frac{4}{9}+\frac{1}{2} x_{u}+\frac{4}{3} \frac{g_{s}^{2}}{g^{2}}, \\
\Delta Z_{l_{R}}^{(1)} & =\frac{1}{c_{\theta}^{2}}-1+\frac{1}{2} x_{l}, & \Delta Z_{d_{R}}^{(1)} & =\frac{1}{9} \frac{1}{c_{\theta}^{2}}-\frac{1}{9}+\frac{1}{2} x_{d}+\frac{4}{3} \frac{g_{s}^{2}}{g^{2}} .
\end{aligned}
$$

Finally, fermion-mass counterterms depend on the sum over the fermion families because of tadpole renormalization (the explicit expression for $\beta_{1}$ can be found in Eq.(82)),

$$
\begin{aligned}
\Delta Z_{m_{l}}^{(1)} & =3 \frac{s_{\theta}^{2}}{c_{\theta}^{2}}+\frac{1}{x_{H}}\left[\frac{3}{2} \frac{1}{c_{\theta}^{4}}+3-2 X_{l}^{2}-6\left(X_{u}^{2}+X_{d}^{2}\right)\right]+\frac{3}{4}\left(x_{H}-x_{l}\right), \\
\Delta Z_{m_{u}^{(1)}} & =\frac{2}{3} \frac{s_{\theta}^{2}}{c_{\theta}^{2}}+\frac{1}{x_{H}}\left[\frac{3}{2} \frac{1}{c_{\theta}^{4}}+3-2 X_{l}^{2}-6\left(X_{u}^{2}+X_{d}^{2}\right)\right]+\frac{3}{4}\left(x_{H}-x_{u}+x_{d}\right)+8 \frac{g_{s}^{2}}{g^{2}}, \\
\Delta Z_{m_{d}}^{(1)} & =-\frac{1}{3} \frac{s_{\theta}^{2}}{c_{\theta}^{2}}+\frac{1}{x_{H}}\left[\frac{3}{2} \frac{1}{c_{\theta}^{4}}+3-2 X_{l}^{2}-6\left(X_{u}^{2}+X_{d}^{2}\right)\right]+\frac{3}{4}\left(x_{H}+x_{u}-x_{d}\right)+8 \frac{g_{s}^{2}}{g^{2}} .
\end{aligned}
$$

\subsection{Finite renormalization}

The last step in one-loop renormalization is the connection between renormalized quantities and POs. Since all quantities at this stage are UV-free, we yerm it finite renormalization. Note that the absorption of UV divergencies into local counterterms is, to some extent, a trivial step; finite renormalization, instead, requires more attention. For example, beyond one loop one cannot use on-shell masses but only complex poles for all unstable particles. The complete formulation of finite renormalization will be given in III. However, let us show some examples where the concept of an on-shell can be employed. Suppose that we renormalize a physical (pseudo-)observable $F$,

$$
F=F_{B}+g^{2} F_{1 L}\left(M^{2}\right)+g^{4} F_{2 L}\left(M^{2}\right),
$$


where $M$ is some renormalized mass which appears at one and two loops in $F_{1_{L}}$ and $F_{2 L}$ but does not show up in the Born term $F_{B}$. In this case we can use the concept of an on-shell mass identifying $M=M_{O S}$ for the two-loop term and performing a finite mass renormalization at one loop,

$$
M^{2}=M_{O S}^{2}\left\{1+\frac{g^{2}}{16 \pi^{2}}\left[\left.\operatorname{Re} \Sigma_{M}^{(1)}\right|_{p^{2}=-M_{O S}^{2}}-\delta Z_{M}^{(1)}\right]\right\}=M_{O S}^{2}+g^{2} \Delta M^{2},
$$

where $M_{O S}$ is the on-shell mass and $\Sigma$ is extracted from the required one-particle irreducible Green function. Eq.(93) is still meaningful (no dependence on gauge parameters) and will be used inside the one-loop result,

$$
F=F_{B}+g^{2} F_{1 L}\left(M_{O S}^{2}\right)+g^{4}\left[F_{2 L}\left(M_{O S}^{2}\right)+F_{1 L}^{\prime}\left(M_{O S}^{2}\right) \Delta M^{2}\right],
$$

where

$$
F_{1 L}^{\prime}\left(M_{O S}^{2}\right)=\left.\frac{\partial F_{1 L}\left(M^{2}\right)}{\partial M^{2}}\right|_{M^{2}=M_{O S}^{2}} .
$$

Here we show some examples of one-loop finite renormalization.

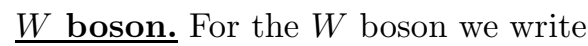

$$
M^{2}=M_{W}^{2}\left[1+\frac{g^{2}}{16 \pi^{2}}\left(\operatorname{Re} \Sigma_{W W}-\delta Z_{M}^{(1)}\right)\right],
$$

where the expression for the counterterm can be read in Eq. (87). The quantity within square brackets in Eq.967) is finite by construction, and it is convenient to write the decomposition

$$
\Sigma_{W W}=\Sigma_{W W}^{f}+\Sigma_{W W}^{b}-2\left(\beta_{1}+\Gamma_{1}\right) .
$$

$\beta_{1}$ and $\Gamma_{1}$ can be read in Eq.821) and Eq.83), whereas the first two terms in the r.h.s. of Eq. (97) refer to fermionic and bosonic contributions. The fermionic part for one generation (one leptonic family, $x_{l}=m_{l}^{2}$ / $M^{2}$, and one up-down quark doublet, $\left.x_{u}=m_{u}^{2} / M^{2}, x_{d}=m_{d}^{2} / M^{2}\right)$ is

$$
\begin{aligned}
\Sigma_{W W}^{f(1)} & =\frac{1}{n-1}\left\{\frac{1}{2}\left(n-2-x_{l}\right) x_{l} a_{0}\left(m_{l}\right)\right. \\
& +\frac{3}{2}\left(x_{u}-x_{d}+n-2\right) x_{d} a_{0}\left(m_{d}\right)+\left(x_{d}-x_{u}+n-2\right) x_{u} a_{0}\left(m_{u}\right) \\
& +\frac{3}{2}\left[(n-1)\left(1-x_{d}-x_{u}\right)+2\left(x_{d}+x_{u}\right)-\left(x_{d}-x_{u}\right)^{2}\right] b_{0}\left(1,1, M^{2}, m_{u}, m_{d}\right) \\
& \left.+\frac{1}{2}\left[(n-1)\left(1-x_{l}\right)+2 x_{l}-x_{l}^{2}-1\right] b_{0}\left(1,1, M^{2}, 0, m_{l}\right)\right\}
\end{aligned}
$$

and the bosonic component reads as follows,

$$
\begin{aligned}
\Sigma_{W W}^{b} & =\frac{1}{4 c_{\theta}^{2}}\left[4(n-2) s_{\theta}^{2}+\frac{1}{n-1} \frac{1}{c_{\theta}^{2}}-4 n-\frac{4}{n-1}+11\right] a_{0}\left(M_{0}\right) \\
& +\frac{1}{4}\left(10-\frac{1}{n-1} \frac{1}{c_{\theta}^{2}}-4 n-\frac{1}{n-1} x_{H}\right) a_{0}(M)+\frac{1}{4}\left(\frac{1}{n-1} x_{H}-1\right) x_{H} a_{0}\left(M_{H}\right) \\
& +\frac{1}{n-1}\left[\frac{1}{4 c_{\theta}^{4}}-\frac{2}{c_{\theta}^{2}}+4+(n-1)\left(-11+4 s_{\theta}^{2}+\frac{2}{c_{\theta}^{2}}\right)\right] b_{0}\left(1,1, M^{2}, M_{0}, M\right) \\
& +\frac{1}{n-1}\left(\frac{x_{H}^{2}}{4}-x_{H}+n-1\right) b_{0}\left(1,1, M^{2}, M, M_{H}\right)-4 s_{\theta}^{2} b_{0}\left(1,1, M^{2}, 0, M\right) .
\end{aligned}
$$

Fermions. For a fermion $f$ we obtain

$$
m_{f}^{2}=m_{f, O S}^{2}\left[1+\frac{g^{2}}{8 \pi^{2}}\left(\Sigma_{f}-\frac{\delta Z_{m, f}^{(1)}}{2}\right)\right]
$$


where the fermion-mass counterterms can be read in Eq.(91), and the physical mass in Eq. (100) coincides with the on-shell one. Using

$$
Q_{l}=-1, Q_{u}=\frac{2}{3}, Q_{d}=-\frac{1}{3}, N_{l}^{c}=1, N_{q}^{c}=3, \quad v_{f}=I_{f}^{(3)}-2 Q_{f} s_{\theta}^{2},
$$

where $I_{f}^{(3)}$ is the third component of isospin, we provide the needed expressions for arbitrary up and down partners in a given fermion doublet. For a generic up-type fermion we get

$$
\begin{aligned}
\Sigma_{u} & =-\frac{1}{8} x_{H} a_{0}\left(M_{H}\right)-\frac{1}{8}\left[1+\frac{1}{x_{u}}\left(n-2+x_{d}\right)\right] a_{0}(M)-\frac{1}{8 c_{\theta}^{2}}\left[1+\frac{1}{x_{u} c_{\theta}^{2}}(n-2)\left(v_{u}^{2}+\frac{1}{4}\right)\right] a_{0}\left(M_{0}\right) \\
& +\frac{1}{8}\left\{(n-2)\left[4 s_{\theta}^{2} Q_{u}^{2}+\frac{1}{c_{\theta}^{2}}\left(v_{u}^{2}+\frac{1}{4}\right)\right]+2 x_{u}\right\} a_{0}\left(m_{u}\right)+\frac{1}{8}\left[1+\frac{1}{x_{u}}\left(n-2+x_{d}\right)\right] x_{d} a_{0}\left(m_{d}\right) \\
& +\frac{1}{8}\left\{n-3+\frac{1}{x_{u}}\left[(n-3) x_{d}-n+2+x_{d}^{2}\right]+x_{u}-2 x_{d}\right\} b_{0}\left(1,1, m_{u}^{2}, M, m_{d}\right) \\
& +\frac{1}{8 c_{\theta}^{2}}\left\{1+(n-2)\left[1-\frac{1}{c_{\theta}^{2} x_{u}}\left(v_{u}^{2}+\frac{1}{4}\right)\right]-4\left(v_{u}^{2}+\frac{1}{4}\right)\right\} b_{0}\left(1,1, m_{u}^{2}, M_{0}, m_{u}\right) \\
& +\frac{1}{8}\left(4 x_{u}-x_{H}\right) b_{0}\left(1,1, m_{u}^{2}, M_{H}, m_{u}\right)-2 s_{\theta}^{2} Q_{u}^{2} b_{0}\left(1,1, m_{u}^{2}, 0, m_{u}\right)-\beta_{1} .
\end{aligned}
$$

For a d-type fermion, the result reads as

$$
\begin{aligned}
\Sigma_{d} & =-\frac{1}{8} x_{H} a_{0}\left(M_{H}\right)-\frac{1}{8}\left[1+\frac{1}{x_{d}}\left(n-2+x_{u}\right)\right] a_{0}(M)-\frac{1}{8 c_{\theta}^{2}}\left[1+\frac{1}{x_{d} c_{\theta}^{2}}(n-2)\left(v_{d}^{2}+\frac{1}{4}\right)\right] a_{0}\left(M_{0}\right) \\
& +\frac{1}{8}\left\{(n-2)\left[4 s_{\theta}^{2} Q_{d}^{2}+\frac{1}{c_{\theta}^{2}}\left(v_{d}^{2}+\frac{1}{4}\right)\right]+2 x_{d}\right\} a_{0}\left(m_{d}\right)+\frac{1}{8}\left[1+\frac{1}{x_{d}}\left(n-2+x_{u}\right)\right] x_{u} a_{0}\left(m_{u}\right) \\
& +\frac{1}{8}\left\{n-3+\frac{1}{x_{d}}\left[(n-3) x_{u}-n+2+x_{u}^{2}\right]+x_{d}-2 x_{u}\right\} b_{0}\left(1,1, m_{d}^{2}, M, m_{u}\right) \\
& +\frac{1}{8 c_{\theta}^{2}}\left\{1+(n-2)\left[1-\frac{1}{c_{\theta}^{2} x_{d}}\left(v_{d}^{2}+\frac{1}{4}\right)\right]-4\left(v_{d}^{2}+\frac{1}{4}\right)\right\} b_{0}\left(1,1, m_{d}^{2}, M_{0}, m_{d}\right) \\
& +\frac{1}{8}\left(\frac{1}{2} x_{d}-x_{H}\right) b_{0}\left(1,1, m_{d}^{2}, M_{H}, m_{d}\right)-2 s_{\theta}^{2} Q_{d}^{2} b_{0}\left(1,1, m_{d}^{2}, 0, m_{d}\right)-\beta_{1} .
\end{aligned}
$$

Higgs boson. We also provide the result for the Higgs-boson mass,

$$
M_{H}^{2}=M_{H, O S}^{2}\left[1+\frac{g^{2}}{16 \pi^{2}}\left(\operatorname{Re} \Sigma_{H H}-\delta Z_{M_{H}}^{(1)}\right)\right],
$$

where the counterterm can be found in Eq.(88), and we use a decomposition similar to Eq.(97), $\Sigma_{H H}=$ $\Sigma_{H H}^{f}+\Sigma_{H H}^{b}$. For one generation of fermions we get

$$
\Sigma_{H H}^{f(1)}=3 \sum_{f=l, u, d} \frac{x_{f}^{2}}{x_{H}} a_{0}\left(m_{f}\right)+\sum_{f=l, u, d} N_{f}^{c} x_{f}\left(\frac{1}{2}-2 \frac{x_{f}}{x_{H}}\right) b_{0}\left(1,1, M_{H}^{2}, m_{f}, m_{f}\right)
$$

whereas the bosonic component reads as follows,

$$
\begin{aligned}
\Sigma_{H H}^{b} & =\frac{1}{x_{H}}\left(n-1+\frac{x_{H}}{2}\right) a_{0}(M)+\frac{1}{c_{\theta}^{2} x_{H}}\left(\frac{n-1}{2 c_{\theta}^{2}}+\frac{x_{H}}{4}\right) a_{0}\left(M_{0}\right)+\frac{3}{4} x_{H} a_{0}\left(M_{H}\right) \\
& -3 \sum_{i=1,3} \sum_{f=l, u, d} N_{f, i}^{c} \frac{x_{f, i}^{2}}{x_{H}} a_{0}\left(m_{f, i}\right)-\left[1-\frac{1}{x_{H}}\left(n-1+\frac{x_{H}^{2}}{4}\right)\right] b_{0}\left(1,1, M_{H}^{2}, M, M\right) \\
& -\left[\frac{1}{2} \frac{1}{c_{\theta}^{2}}-\frac{1}{x_{H}}\left(\frac{n-1}{2 c_{\theta}^{4}}+\frac{x_{H}^{2}}{8}\right)\right] b_{0}\left(1,1, M_{H}^{2}, M_{0}, M_{0}\right)+\frac{9}{8} x_{H}^{2} b_{0}\left(1,1, M_{H}^{2}, M_{H}, M_{H}\right) .
\end{aligned}
$$

The sum over the fermion masses in Eq.(106) depends on the $\beta_{1}$ parameter (see Eq.(82)), which we included explicitly in the computation of the Higgs-boson self-energy. 


\subsection{Ward-Slavnov-Taylor identities}

Ward-Slavnov-Taylor (WST) identities play a crucial role in showing that the electroweak theory is renormalizable and respects unitarity at the same time. In fact, we can prove with their help that transition amplitudes are the same in every non-singular gauge. Having derived the one-loop counterterms, we analyze how renormalization affects the WST identities of the theory; note that here we consider only a particular class of WST identities, the so-called doubly-contracted ones with two external gauge-boson fields. Let us consider the gauge-fixing functions $\mathcal{C}^{a}$ of Eq.(58) written in terms of bare quantities,

$$
\mathcal{C}^{A}=-\frac{1}{\xi_{A}^{b}} \partial_{\mu} A_{\mu}^{b}-\xi_{A Z}^{b} \partial_{\mu} Z_{\mu}^{b}, \quad \mathcal{C}^{Z}=-\frac{1}{\xi_{Z}^{b}} \partial_{\mu} Z_{\mu}^{b}+\xi_{\varphi^{0}}^{b} M_{0}^{b} \varphi^{b, 0}, \quad \mathcal{C}^{ \pm}=-\frac{1}{\xi_{W}^{b}} \partial_{\mu} W_{\mu}^{b, \pm}+\xi_{\varphi}^{b} M^{b} \varphi^{b, \pm}
$$

Then, we introduce renormalized quantities and we set the renormalized gauge parameters to unity,

$$
\begin{aligned}
& \mathcal{C}^{A}=-Z_{A A}^{\frac{1}{2}} Z_{\xi_{A}}^{-1} \partial_{\mu} A_{\mu}-\left(Z_{A Z}^{\frac{1}{2}} Z_{\xi_{A}}^{-1}+Z_{Z}^{\frac{1}{2}} Z_{\xi_{A Z}}\right) \partial_{\mu} Z_{\mu}, \\
& \mathcal{C}^{Z}=-Z_{Z}^{\frac{1}{2}} Z_{\xi_{Z}}^{-1} \partial_{\mu} Z_{\mu}+Z_{\varphi^{0}}^{\frac{1}{2}} Z_{M}^{\frac{1}{2}} Z_{c_{\theta}}^{-1} Z_{\xi_{\varphi} 0} M_{0} \varphi^{0}, \\
& \mathcal{C}^{ \pm}=-Z_{W}^{\frac{1}{2}} Z_{\xi_{W}}^{-1} \partial_{\mu} W_{\mu}^{ \pm}+Z_{\varphi}^{\frac{1}{2}} Z_{M}^{\frac{1}{2}} Z_{\xi_{\varphi}} M \varphi^{ \pm} .
\end{aligned}
$$

Next, we associate a set of source vertices to the Fourier transform of a gauge-fixing function $\mathcal{C}^{a}$.

Let us consider $\mathcal{C}^{ \pm}$, where we get the two source vertices of Fig. 1. A doubly-contracted two-point WST identity is obtained by connecting two sources through vertices and propagators. Here we get, at every order in perturbation theory, the identity of Fig. 2. We analyze the WST identity at $\mathcal{O}\left(g^{2}\right)$. After expanding

$$
\begin{aligned}
& \overbrace{p}^{W}-(2 \pi)^{4} i i_{\mu} Z_{W}^{1 / 2} Z_{\xi_{W}}^{-1}
\end{aligned}
$$

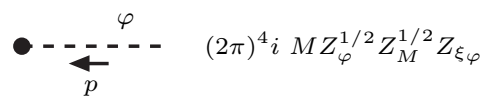

Figure 1: Sources related to the gauge-fixing functions $\mathcal{C}^{ \pm}$defined in Eq.(108). The momentum $p$ is flowing inwards.

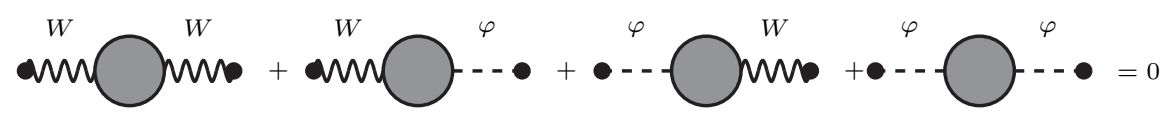

Figure 2: Doubly-contracted WST identity with two external $\mathcal{C}^{ \pm}$sources. Gray circles contain all the irreducible and reducible Feynman diagrams contributing to the needed Green functions. Black dotted circles represent sources. Their expression can be read in Fig. 1]

the renormalization constants $Z_{i}$ up to $\mathcal{O}\left(g^{2}\right)$ we obtain

$$
\mathcal{C}^{ \pm}=-\left[1+\frac{g^{2}}{16 \pi^{2}}\left(\frac{1}{2} \delta Z_{W}^{(1)}-\delta Z_{\xi_{W}}^{(1)}\right)\right] \partial_{\mu} W_{\mu}^{ \pm}+\left[1+\frac{g^{2}}{16 \pi^{2}}\left(\frac{1}{2} \delta Z_{\varphi}^{(1)}+\frac{1}{2} \delta Z_{M}^{(1)}+\delta Z_{\xi_{\varphi}}^{(1)}\right)\right] M \varphi^{ \pm} .
$$

and we get the source terms of Fig. 3. As a consequence, the doubly-contracted WST identity with two external $\mathcal{C}^{ \pm}$sources at $\mathcal{O}\left(g^{2}\right)$ of Fig. 2 receives two different contributions, whose graphical representations

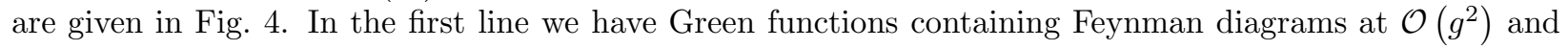


source vertices at $\mathcal{O}(1)$. The second line, instead, contains Green functions with Feynman diagrams at $\mathcal{O}(1)$ and source vertices at $\mathcal{O}\left(g^{2}\right)$. Finally we use Eq.(85),

$$
\delta Z_{\xi_{W}}^{(1)}=\frac{1}{2} \delta Z_{W}^{(1)}, \quad \delta Z_{\xi_{\varphi}}^{(1)}=-\frac{1}{2}\left(\delta Z_{\varphi}^{(1)}+\delta Z_{M}^{(1)}\right),
$$

and we are immediately left with the four contributions displayed in the first line of Fig. 4. Note that this is exactly the doubly-contracted identity with two external $\mathcal{C}^{ \pm}$-source vertices which appears in the 't Hooft-Feynman gauge. In order to get the complete set of doubly-contracted WST identities we insert the

$$
\underbrace{W}_{\mu}-(2 \pi)^{4} i i p_{\mu}
$$

Figure 3: Source vertices related to the gauge-fixing functions $\mathcal{C}^{ \pm}$. We start from Fig. 1 and expand the renormalization constants up to $\mathcal{O}\left(g^{2}\right)$. The black squares denote source vertices at $\mathcal{O}(1)$, whereas the white squares denote source vertices at $\mathcal{O}\left(g^{2}\right)$.

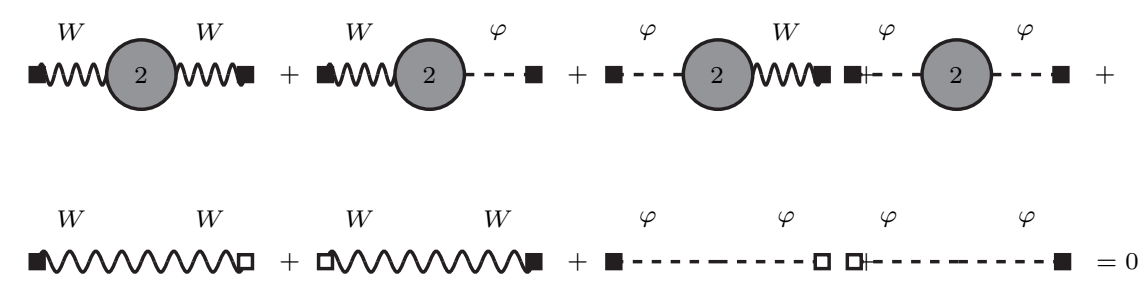

Figure 4: Doubly-contracted WST identity with two external $\mathcal{C}^{ \pm}$sources at $\mathcal{O}\left(g^{2}\right)$. Gray circles contain all the irreducible and reducible Feynman diagrams contributing to the Green functions at $\mathcal{O}\left(g^{2}\right)$. Black squares and white squares represent source vertices. Their expression can be read in Fig. 3

explicit expressions for the one-loop counterterms of Eq.(85) in Eq.(108). Besides Eq.(110) we get

$$
\delta Z_{\xi_{A}}^{(1)}=\frac{1}{2} \delta Z_{A A}^{(1)}, \quad \delta Z_{\xi_{A Z}}^{(1)}=-\delta Z_{A Z}^{(1)}, \quad \delta Z_{\xi_{Z}}^{(1)}=\frac{1}{2} \delta Z_{Z}^{(1)}, \quad \delta Z_{\xi_{\varphi^{0}}}^{(1)}=\delta Z_{c_{\theta}}^{(1)}-\frac{1}{2}\left(\delta Z_{\varphi^{0}}^{(1)}+\delta Z_{M}^{(1)}\right) .
$$

Using Eq.(110) and Eq.(111) in Eq.(108) we finally obtain

$$
\mathcal{C}^{A}=-\partial_{\mu} A_{\mu}+\mathcal{O}\left(g^{4}\right), \quad \mathcal{C}^{Z}=-\partial_{\mu} Z_{\mu}+M_{0} \varphi^{0}+\mathcal{O}\left(g^{4}\right), \quad \mathcal{C}^{ \pm}=-\partial_{\mu} W_{\mu}^{ \pm}+M \varphi^{ \pm}+\mathcal{O}\left(g^{4}\right) .
$$

The whole set of one-loop WST identities is given in Fig. 5, whereas source terms can be read in Fig. 6. The results are exactly the doubly-contracted identities with two external source vertices in 't Hooft-Feynman gauge. 


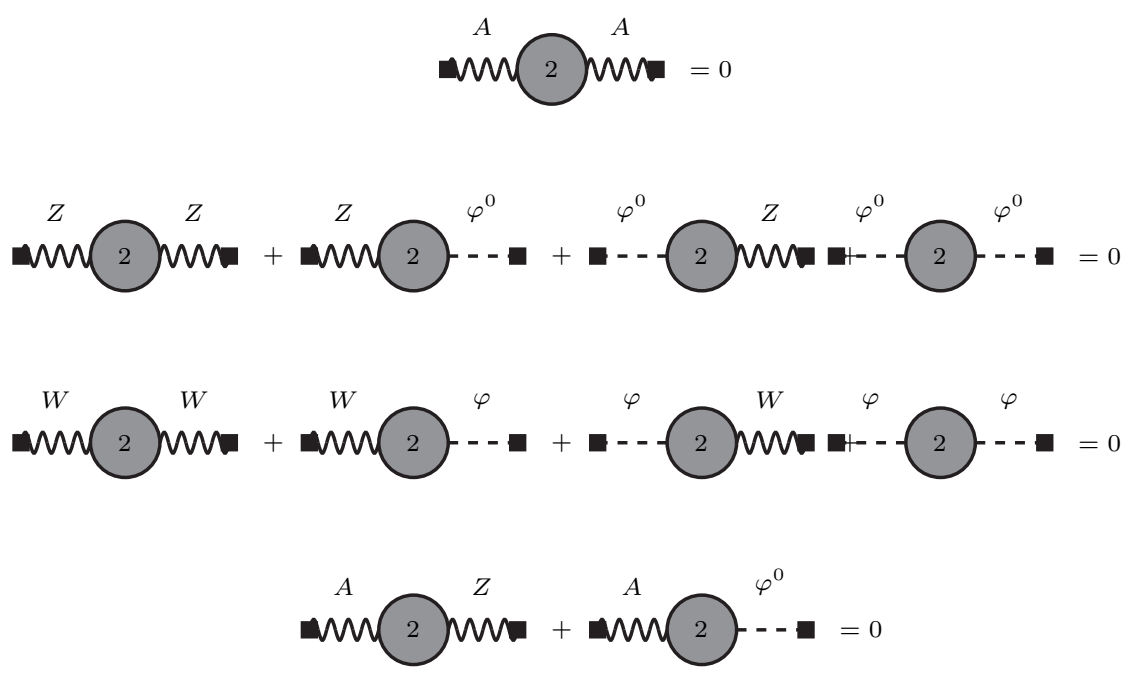

Figure 5: Doubly-contracted WST identities with two external gauge bosons at $\mathcal{O}\left(g^{2}\right)$. Gray circles denote the sum of the needed Feynman diagrams at $\mathcal{O}\left(g^{2}\right)$. Source vertices are given in Fig. 6 .

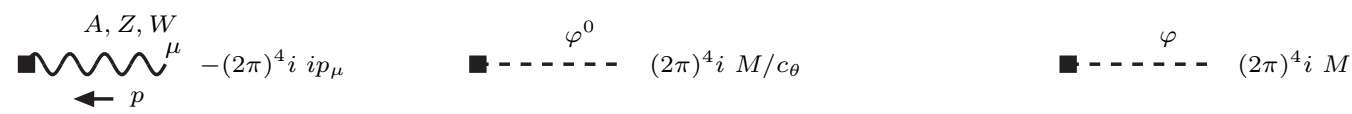

Figure 6: Renormalized source vertices for the WST identities of Fig. 5

\section{$6 \quad \beta$ and $\Gamma$ at two loops}

The first step in extending our renormalization procedure beyond one loop has to do, once again, with tadpole renormalization $(\beta$, Subsections 6.1) and 6.2) and neutral-sector diagonalization $(\Gamma$, Subsections 6.3 and 6.4).

\section{1 $\beta_{2}$ : reducible contributions}

We consider the contributions of Fig. 7 to the reducible Green function with one external Higgs boson at $\mathcal{O}\left(g^{3}\right)$

$$
G_{\mathrm{red}}^{H ; 2}=\sum_{i=1}^{9} F_{i ; \mathrm{red}}^{H ; 2},
$$

where the sum runs over the nine families of Feynman diagrams of Fig. 7 . The sum over the first six families is given by

$$
\sum_{i=1}^{6} F_{i ; \text { red }}^{H ; 2}=\left[\sum_{j=1}^{3} F_{j ; \text { irr }}^{H H ; 1}\right] \frac{1}{(2 \pi)^{4} i} \frac{1}{p^{2}+M_{H}^{2}}\left[\sum_{k=1}^{2} F_{k ; \mathrm{irr}}^{H ; 1}\right]
$$

where $F_{j ; \text { irr }}^{H H ; 1}$ denotes one of the three families of Feynman diagrams which contribute to the Green function with two external Higgs bosons at $\mathcal{O}\left(g^{2}\right)$ and $F_{k ; \text { irr }}^{H ; 1}$ is one of the two families of Feynman diagrams which contribute to the Green function with one external Higgs boson at $\mathcal{O}(g)$. As a consequence of our choice for 


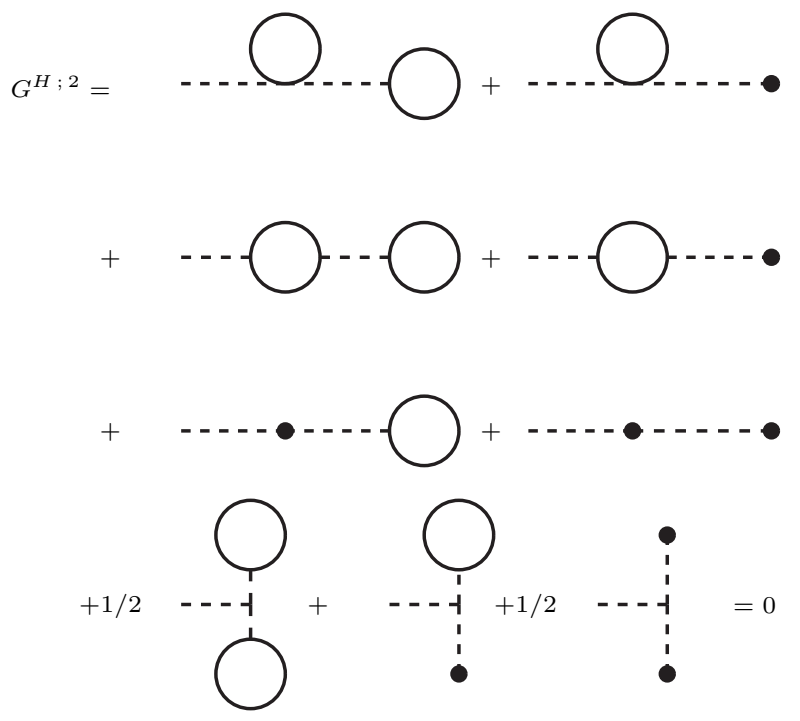

Figure 7: The reducible contributions to the renormalization of the Higgs-boson vacuum expectation value at $\mathcal{O}\left(g^{3}\right)$. Nine families of Feynman diagrams give a contribution. Dashed lines represent a Higgs boson. Dotted one-leg vertices are $\beta_{1}$-dependent vertices at $\mathcal{O}(g)$. Dotted two-leg vertices, instead, depend on $\beta_{1}$ and on one-loop counterterms. Combinatorial factors for two-loop reducible diagrams are given by the products of those for one-loop diagrams, except for two cases, where we indicated explicitly the additional multiplicative factor.

$\beta_{1}$ we get

$$
F_{1 ; \text { irr }}^{H ; 1}+F_{2 ; \text { irr }}^{H ; 1}=0 \quad \rightarrow \quad \sum_{i=1}^{6} F_{i ; \text { red }}^{H ; 2}=0 .
$$

For the last three diagrams of Fig. 7 we obtain another vanishing component,

$$
\sum_{i=7}^{9} F_{i ; \text { red }}^{H ; 2}=-\frac{1}{(2 \pi)^{4} i} \frac{3}{4} \frac{g M_{H}^{2}}{M} \frac{1}{\left(p^{2}+M_{H}^{2}\right)^{2}}\left[F_{1 ; \text { irr }}^{H ; 1}+F_{2 ; \text { irr }}^{H ; 1}\right]^{2}=0 .
$$

As a result, $\beta_{1}$ receives contributions only from irreducible diagrams.

\section{2 $\beta_{2}$ : irreducible contributions}

In order to derive $\beta_{2}$ we take in account only irreducible diagrams and we write

$$
G_{\mathrm{irr}}^{H ; 2}=\sum_{j=1}^{6} F_{j ; \mathrm{irr}}^{H ; 2}=0
$$

where the sum is over the six families of irreducible diagrams of Fig. 8 . Note that here we are dealing with three kinds of diagrams. The first three families contain two-loop diagrams at $\mathcal{O}\left(g^{3}\right)$. The fourth and the fifth family contain one-loop diagrams obtained with the insertion of a dotted vertex which represent a $\beta_{1}, \Gamma_{1}$ or one-loop counterterm insertion. Explicit expressions for $\beta_{1}$ and $\Gamma_{1}$ can be read respectively in Eqs.(82) and (83). One-loop counterterms are given in Eqs.(85)-(88), Eqs.(89)-(90) and Eq.(91). The last family contains one diagram with a $\beta_{2}$-dependent vertex. Here we present the UV divergent residue for $\beta_{2}$ 


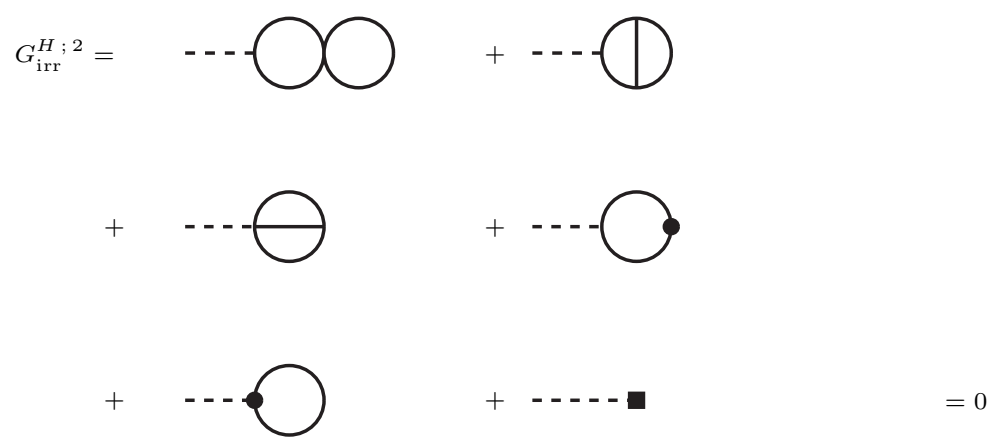

Figure 8: The irreducible contributions to the renormalization of the Higgs-boson vacuum expectation value at $\mathcal{O}\left(g^{3}\right)$. Six families of Feynman diagrams give a contribution. Dotted vertices depend on $\beta_{1}, \Gamma_{1}$ or the one-loop counterterms. The black-square vertex, instead, represents a $\beta_{2}$-dependent vertex.

neglecting the fermion masses, except for the top-quark mass $m_{t}$. Although not reported in this paper, the full result is available. As usual, we write the decomposition

$$
\beta_{2}=\left(\frac{1}{\epsilon}-\Delta_{U V}\right)\left(\frac{\beta_{2 ; U V, 1}}{\epsilon}+\beta_{2 ; U V, 2}\right)+\Delta_{U V}^{2} \beta_{2 ; U V, 3}+\beta_{2 ; F} .
$$

The expression for $\beta_{2 ; U V, 1}$ is given by

$$
\begin{aligned}
\beta_{2 ; U V, 1} & =\frac{1}{128 \pi^{4}}\left[\frac{9}{x_{H}^{2}}\left(-\frac{1}{16 c_{\theta}^{8}}+\frac{1}{2} \frac{x_{t}^{2}}{c_{\theta}^{4}}-\frac{1}{4 c_{\theta}^{4}}+x_{t}^{2}-x_{t}^{4}-\frac{1}{4}\right)\right. \\
& +\frac{1}{2 x_{H}}\left(\frac{185}{8} \frac{1}{c_{\theta}^{6}}-\frac{9}{2} \frac{x_{t}}{c_{\theta}^{4}}-\frac{153}{4} \frac{1}{c_{\theta}^{4}}+\frac{13}{2} \frac{x_{t}^{2}}{c_{\theta}^{2}}+\frac{65}{4} \frac{1}{c_{\theta}^{2}}-9 x_{t}-x_{t}^{2}-9 x_{t}^{3}-\frac{27}{2}\right) \\
& +\frac{1}{4}\left(-\frac{53}{12}-\frac{11}{16} \frac{1}{c_{\theta}^{4}}-\frac{43}{12} \frac{1}{c_{\theta}^{2}}+\frac{3}{2} \frac{x_{t}}{c_{\theta}^{2}}+3 x_{t}\right)+\frac{x_{H}}{8}\left(-\frac{15}{4} \frac{1}{c_{\theta}^{2}}+9 x_{t}-\frac{25}{2}\right) \\
& \left.+\frac{45}{64} x_{H}^{2}+48 \frac{g_{S}^{2}}{g^{2}} \frac{x_{t}^{2}}{x_{H}}\right] .
\end{aligned}
$$

The expression for $\beta_{2 ; U V, 2}$ reads as

$$
\begin{aligned}
\beta_{2 ; U V, 2} & =\frac{1}{128 \pi^{4}}\left\{\frac{3}{x_{H}^{2}}\left(\frac{1}{16 c_{\theta}^{8}}-\frac{x_{t}^{2}}{c_{\theta}^{4}}+\frac{1}{4 c_{\theta}^{4}}-2 x_{t}^{2}+3 x_{t}^{4}+\frac{1}{4}\right)\right. \\
& +\frac{1}{x_{H}}\left(-\frac{47}{6} \frac{1}{c_{\theta}^{6}}-\frac{19}{8} \frac{x_{t}}{c_{\theta}^{4}}+\frac{287}{24} \frac{1}{c_{\theta}^{4}}+10 \frac{x_{t}}{c_{\theta}^{2}}-\frac{2}{3} \frac{x_{t}^{2}}{c_{\theta}^{2}}-\frac{151}{24} \frac{1}{c_{\theta}^{2}}-\frac{35}{4} x_{t}+\frac{19}{6} x_{t}^{2}\right. \\
& \left.+\frac{15}{4} x_{t}^{3}+\frac{127}{6}\right)+\frac{1}{2}\left(\frac{9}{4} \frac{1}{c_{\theta}^{4}}-\frac{3}{8} \frac{x_{t}}{c_{\theta}^{2}}-\frac{23}{24} \frac{1}{c_{\theta}^{2}}-\frac{3}{4} x_{t}-3 x_{t}^{2}+\frac{143}{24}\right)+\frac{x_{H}}{16}\left(\frac{3}{c_{\theta}^{2}}-9 x_{t}+1\right)-\frac{3}{8} x_{H}^{2} \\
& +\frac{1}{4} \frac{\ln c_{\theta}^{2}}{c_{\theta}^{2}}\left[9 \frac{1}{x_{H}^{2} c_{\theta}^{2}}\left(\frac{1}{4 c_{\theta}^{4}}-x_{t}^{2}+\frac{1}{2}\right)+\frac{1}{x_{H}}\left(-\frac{13}{4} \frac{1}{c_{\theta}^{2}}-\frac{3}{2} x_{t}^{2}+\frac{3}{4}\right)\right. \\
& \left.+\frac{1}{8}\left(\frac{17}{2} \frac{1}{c_{\theta}^{2}}-\frac{13}{3}\right)+\frac{3}{16} x_{H}\right]+\frac{1}{8} \ln x_{H}\left[-\frac{9}{2}-\frac{9}{4} \frac{1}{c_{\theta}^{4}}+\frac{3}{8} \frac{x_{H}}{c_{\theta}^{2}}+\frac{13}{4} x_{H}-\frac{9}{8} x_{H}^{2}+9 x_{t}^{2}\right] \\
& \left.+x_{t}^{2} \ln x_{t}\left[\frac{9}{4} \frac{1}{c_{\theta}^{4} x_{H}^{2}}-\frac{3}{8} \frac{1}{c_{\theta}^{2} x_{H}}+\frac{9}{2} \frac{1}{x_{H}^{2}}-9 \frac{x_{t}^{2}}{x_{H}^{2}}-\frac{13}{4} \frac{1}{x_{H}}+\frac{9}{8}\right]-8 \frac{g_{S}^{2}}{g^{2}} \frac{x_{t}^{2}}{x_{H}}\right\} .
\end{aligned}
$$


Now, the expression for $\beta_{2 ; U V, 3}$ is given by

$$
\begin{aligned}
\beta_{2 ; U V, 3} & =\frac{1}{128 \pi^{4}}\left[\frac{1}{x_{H}}\left(\frac{185}{64} \frac{1}{c_{\theta}^{6}}-\frac{9}{16} \frac{x_{t}}{c_{\theta}^{4}}+\frac{13}{16} \frac{x_{t}^{2}}{c_{\theta}^{2}}+\frac{17}{32} \frac{1}{c_{\theta}^{2}}-\frac{79}{16} \frac{1}{c_{\theta}^{4}}-\frac{9}{8} x_{t}+\frac{x_{t}^{2}}{2}-\frac{9}{8} x_{t}^{3}-5\right)\right. \\
& +\frac{1}{16}\left(-1+\frac{3}{2} \frac{1}{c_{\theta}^{4}}-\frac{17}{4} \frac{1}{c_{\theta}^{2}}+3 x_{t}-9 x_{t}^{2}+\frac{3}{2} \frac{x_{t}}{c_{\theta}^{2}}\right)+\frac{3}{32} x_{H}\left(-\frac{5}{4} \frac{1}{c_{\theta}^{2}}+3 x_{t}-5\right) \\
& \left.+\frac{27}{128} x_{H}^{2}+12 \frac{g_{S}^{2}}{g^{2}} \frac{x_{t}^{2}}{x_{H}}\right] .
\end{aligned}
$$

It is worth nothing that, as a consequence of our choice for $\beta_{2}$, reducible Feynman diagrams containing a
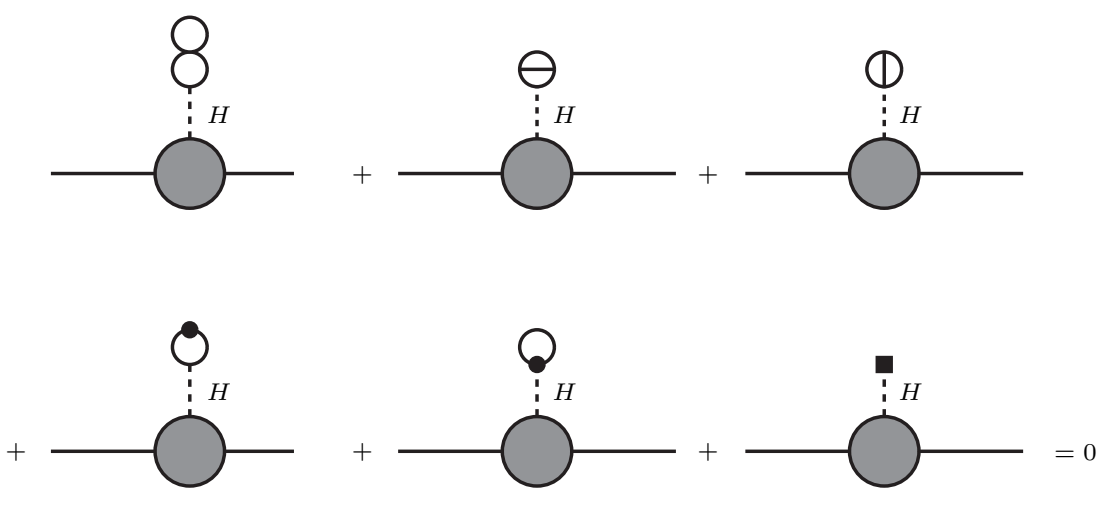

Figure 9: $\mathcal{O}\left(g^{3}\right)$ tadpole insertions contributing to the reducible two-point Green function at $\mathcal{O}\left(g^{k}\right)$. The gray circle denotes the three-point Green function, with an additional Higgs boson line, at $\mathcal{O}\left(g^{k-3}\right)$. These diagrams cancel as a consequence of our choice for $\beta_{2}$. Dotted and squared vertices are the same introduced in Fig. 8 .

tadpole subdiagram at $\mathcal{O}(g)^{3}$ should not be included in any computation (this is displayed in Fig. 9).

\section{3 $\quad \Gamma_{2}$ : reducible contributions}

Let us start with the reducible contributions of Fig. 10, whose contribution to the Green function with one

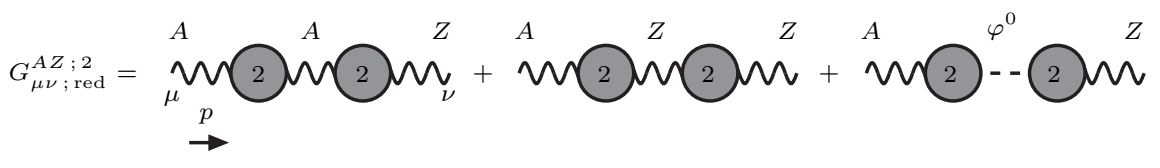

Figure 10: Reducible contributions to the Green function with one external photon and one external $Z$ boson at $\mathcal{O}\left(g^{4}\right)$. Gray circles denote the sum over the irreducible Feynman diagrams at $\mathcal{O}\left(g^{2}\right)$.

external photon and one external $Z$ boson at $\mathcal{O}\left(g^{4}\right)$ can be written through the two-point Green functions 
at one loop involving neutral gauge bosons,

$$
G_{\mu \nu ; \operatorname{red}}^{A Z ; 2}=\frac{1}{(2 \pi)^{4} i}\left\{G_{\mu \alpha ; \operatorname{irr}}^{A A ; 1} \frac{1}{p^{2}} G_{\alpha \nu ; \operatorname{irr}}^{A Z ; 1}+G_{\mu \alpha ; \operatorname{irr}}^{A Z ; 1} \frac{1}{p^{2}+M_{0}^{2}} G_{\alpha \nu ; \operatorname{irr}}^{Z Z ; 1}+G_{\mu ; \operatorname{irr}}^{A \varphi^{0} ; 1} \frac{1}{p^{2}+M_{0}^{2}} G_{\nu ; \operatorname{irr}}^{\varphi^{0} Z ; 1}\right\} .
$$

We extract form factors as

$$
\begin{aligned}
& G_{\mu \nu}^{i j ; 2}=G_{d}^{i j ; 2}\left(p^{2}\right) \delta_{\mu \nu}+G_{p p}^{i j ; 2}\left(p^{2}\right) p_{\mu} p_{\nu} \quad(i, j \text { vectors }) \\
& G_{\mu}^{i j ; 2}=G_{p}^{i j ; 2}\left(p^{2}\right) i p_{\mu} \quad(i \text { vector, } \quad j \quad \text { scalar })
\end{aligned}
$$

and obtain

$$
G_{d ; \text { red }}^{A Z ; 2}\left(p^{2}\right)=\frac{1}{(2 \pi)^{4} i} G_{d ; \text { irr }}^{A Z ; 1}\left(p^{2}\right)\left\{G_{d ; \text { irr }}^{A A ; 1}\left(p^{2}\right) \frac{1}{p^{2}}+G_{d ; \text { irr }}^{Z Z ; 1}\left(p^{2}\right) \frac{1}{p^{2}+M_{0}^{2}}\right\} .
$$

The photon self-energy shows factorization,

$$
G_{d ; \text { irr }}^{A A ; 1}\left(p^{2}\right)=p^{2} \Pi_{d}^{A A ; 1}\left(p^{2}\right) .
$$

It follows that Eq.(124), at $p^{2}=0$, can be written as

$$
G_{d ; \text { red }}^{A Z ; 2}(0)=\frac{1}{(2 \pi)^{4} i} G_{d ; \text { irr }}^{A Z ; 1}(0)\left\{\Pi_{d}^{A A ; 1}(0)+G_{d ; \text { irr }}^{Z Z ; 1}(0) \frac{1}{M_{0}^{2}}\right\} .
$$

Following our choice for $\Gamma_{1}$ we have $G_{d ; \text { irr }}^{A Z ; 1}(0)=0$. As a consequence, the reducible contributions of Eq.(124) vanish. Therefore, $\Gamma_{2}$ is fixed through irreducible Feynman diagrams,

$$
G_{d}^{A Z ; 2}(0)=G_{d ; \text { irr }}^{A Z ; 2}(0)=0 .
$$

\section{4 $\Gamma_{2}$ : irreducible contributions}

To fix $\Gamma_{2}$ we use

$$
G_{d ; \text { irr }}^{A Z ; 2}(0)=\sum_{i=1}^{13} F_{d ; i ; \operatorname{irr}}^{A Z ; 2}(0)=0,
$$

where the sum is over the form factors of the thirteen families of irreducible diagrams of Fig. 14. Here we get three kinds of diagrams. The first eight families contain two-loop diagrams at $\mathcal{O}\left(g^{4}\right)$. Next, we have four families of one-loop diagrams obtained by inserting a vertex which depends on $\beta_{1}, \Gamma_{1}$, or on a one-loop counterterm. Note that these diagrams depend also on the counterterms for the gauge parameters $\delta Z_{\xi_{i}}$. Obviously, there will be no track of the gauge parameters in the final results since we made the identification $\xi_{i}=1$. Nevertheless, gauge-parameter counterterms play an essential role in making every Green function UV-finite. Finally, the last family contains a single Feynman diagram with a $\Gamma_{2}$-dependent vertex.

The contribution of the thirteenth family of Fig. 14 is given by

$$
\begin{aligned}
F_{d ; 13 ; \operatorname{irr}}^{A Z ; 2}(0) & =-(2 \pi)^{4} i g^{4} M^{2} s_{\theta} / c_{\theta}\left\{\Gamma_{2}+\left(c_{\theta}^{2} \Gamma_{1}^{2}+2 \Gamma_{1} \beta_{1}\right)\right. \\
& \left.+\frac{\Gamma_{1}}{16 \pi^{2}}\left[\delta Z_{s_{\theta}}^{(1)}-\delta Z_{c_{\theta}}^{(1)}+2 \delta Z_{g}^{(1)}+\delta Z_{M}^{(1)}+\frac{1}{2}\left(\delta Z_{A A}^{(1)}+\delta Z_{Z}^{(1)}\right)\right]\right\} .
\end{aligned}
$$

Using Eq.(129) in Eq.(128) we get

$$
\begin{aligned}
\Gamma_{2} & =\frac{1}{(2 \pi)^{4} i} \frac{c_{\theta} \sum_{i=1}^{12} F_{d \mid 12 ; \mathrm{irr}}^{A Z ;}(0)}{g^{4} s_{\theta} M^{2}}-\left(c_{\theta}^{2} \Gamma_{1}^{2}+2 \Gamma_{1} \beta_{1}\right) \\
& -\frac{\Gamma_{1}}{16 \pi^{2}}\left[\delta Z_{s_{\theta}}^{(1)}-\delta Z_{c_{\theta}}^{(1)}+2 \delta Z_{g}^{(1)}+\delta Z_{M}^{(1)}+\frac{1}{2}\left(\delta Z_{A A}^{(1)}+\delta Z_{Z}^{(1)}\right)\right] .
\end{aligned}
$$


$\Gamma_{2}$ can be expressed through a linear combination of products of one- and two-loop scalar integrals, evaluated at zero-momentum transfer. As usual, we write the decomposition

$$
\Gamma_{2}=\left(\frac{1}{\epsilon^{2}}-\Delta_{U V}\right)\left(\frac{\Gamma_{2 ; U V, 1}}{\epsilon}+\Gamma_{2 ; U V, 2}\right)+\Delta_{U V}^{2} \Gamma_{2 ; U V, 3}+\Gamma_{2 ; F},
$$

where the UV residues are

$$
\Gamma_{2 ; U V, 1}=\frac{169}{1536 \pi^{4}}, \quad \Gamma_{2 ; U V, 2}=-\frac{137}{6144 \pi^{4}}, \quad \Gamma_{2 ; U V, 3}=\frac{111}{2048 \pi^{4}} .
$$

\section{Two-loop self-energies}

In this section we will show that two-loop self-energies develop UV poles whose residues are polynomials in the external momentum (local residues). Therefore, a suitable choice for the counterterms in the $\overline{M S}$ scheme removes UV divergencies also at two loops. We generate the needed Feynman diagrams with $\mathcal{G}$ raphShot 8 ] and express every two-loop two-point Green function through scalar integrals using standard tensor-reduction techniques. Finally, we extract UV residues by means of the results collected in Section 2

In Subsection 7.1 we introduce our notations, and in Subsection 7.2 and Subsection 7.3 we review the transverse form factors for the photon self-energy and the transition between the photon and the $Z$ boson. Explicit results for the transverse components of the $Z$ - and $W$-boson self-energies and for the Higgs-boson self-energy are shown in Subsection 7.4. Subsection 7.5 and Subsection 7.6.

\subsection{Notation}

Reducible two-loop two-point Green functions with two external boson lines are displayed in Fig. [1]. They are UV finite by construction, since one-loop counterterms have already been fixed. Therefore, we can limit our discussion to irreducible two-loop two-point Green functions, which can be written through the form factors introduced in Eq.(123),

$$
G_{a ; \text { irr }}^{i j ; 2}(s)=\sum_{k=1}^{13} F_{a ; \operatorname{irr} ; k}^{i j ; 2}(s) .
$$

Here $i$ and $j$ are the external fields, the subscript $a$ labels the form factor and the sum runs over the thirteen families of Feynman diagrams of Fig. 14. We shall organize the list of results according to the following decomposition:

$$
G_{a ; \text { irr }}^{i j ; 2}(s)=G_{a ; \text { irr }}^{i j ; 2 \text { bos }}(s)+G_{a ; \operatorname{irr}}^{i j ; 2 ; \text { lep }}(s)+G_{a ; \text { irr }}^{i j ; 2 ; l q}(s)+G_{a ; \text { irr }}^{i j ; 2 ; t b}(s),
$$

where we split the complete result into purely-bosonic diagrams (bos), diagrams with one lepton sub-loop (lep), diagrams with one light-quark sub-loop (lq; $u, d, c$ and $s$ ) and diagrams with one top-bottom sub-loop (tb). Furthermore, we extract UV poles using Eq. (799) and we always leave out an overall factor $(2 \pi)^{4} i$. In the following we show results neglecting the masses of the leptons and the five light quarks. Note, however, that the full result is available.

\subsection{The photon self-energy}

When computing the photon self-energy at a given order in perturbation theory we adopt the convention of excluding diagrams which contain light quarks coupled to photons or gluons with no other hard scale. In this case the correct treatment requires the use of non-perturbative dispersion relations [23]. So there are two preliminary steps: first, we exclude from the computation two-loop diagrams which contain only light quarks and photons (or gluons). Next, we derive again one-loop counterterms for light-quark fields and masses neglecting one-loop diagrams which contain only light quarks and photons (or gluons). The results 


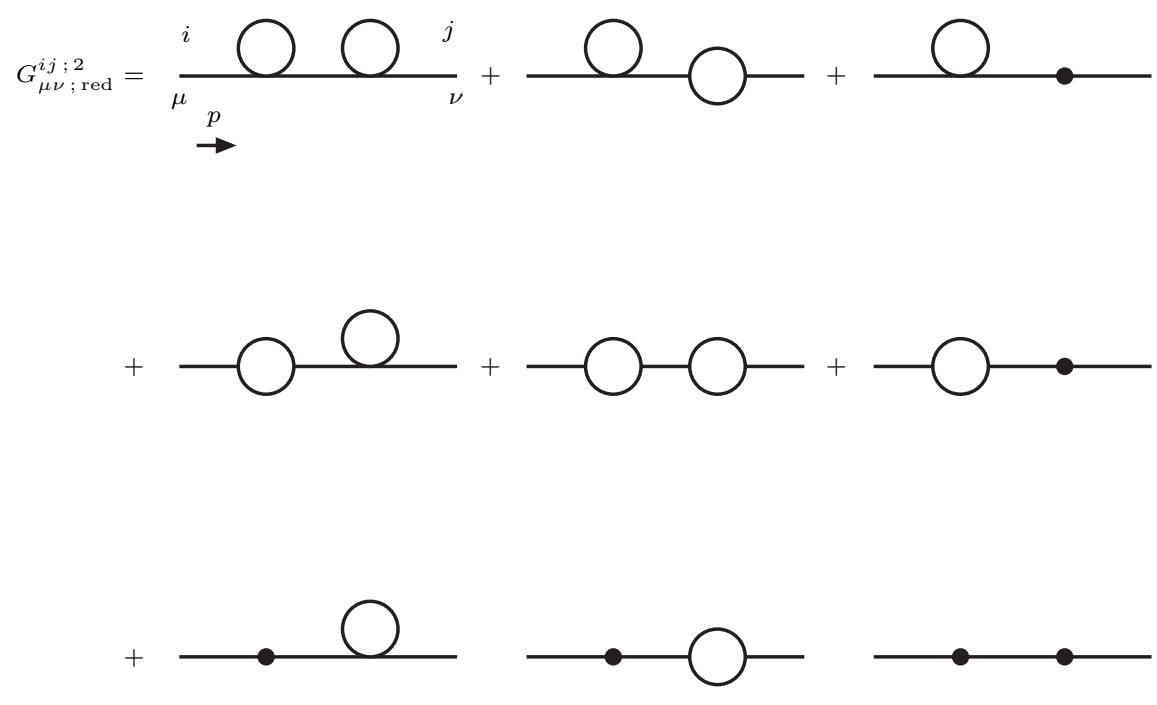

Figure 11: Reducible contributions to the two-point Green function with external boson fields $i$ and $j$ at $\mathcal{O}\left(g^{4}\right)$. The dotted two-leg vertex represents a $\beta_{1^{-}}, \Gamma_{1^{-}}$or one-loop-counterterm-dependent vertex.

for up- and down-quark fields reads as

$$
\begin{array}{rlrl}
\Delta Z_{u_{L}}^{(1)} & =\frac{1}{4}\left(\frac{1}{9} \frac{1}{c_{\theta}^{2}}+\frac{10}{9}+\frac{16}{9} c_{\theta}^{2}+x_{u}+x_{d}\right), & \Delta Z_{d_{L}}^{(1)}=\frac{1}{4}\left(\frac{1}{9} \frac{1}{c_{\theta}^{2}}+\frac{22}{9}+\frac{4}{9} c_{\theta}^{2}+x_{u}+x_{d}\right), \\
\Delta Z_{u_{R}}^{(1)}=\frac{1}{4}\left(\frac{16}{9} \frac{1}{c_{\theta}^{2}}-\frac{32}{9}+\frac{16}{9} c_{\theta}^{2}+2 x_{u}\right), & \Delta Z_{d_{R}}^{(1)}=\frac{1}{4}\left(\frac{4}{9} \frac{1}{c_{\theta}^{2}}-\frac{8}{9}+\frac{4}{9} c_{\theta}^{2}+2 x_{d}\right) .
\end{array}
$$

Light-quark mass counterterms are given by

$$
\begin{aligned}
\Delta Z_{m_{u}}^{(1)} & =-\frac{1}{3}\left(-2 \frac{1}{c_{\theta}^{2}}+10-8 c_{\theta}^{2}\right)+\frac{3}{x_{H}}\left[\frac{1}{2} \frac{1}{c_{\theta}^{4}}+1-2\left(X_{u}^{2}+X_{d}^{2}\right)-\frac{2}{3} X_{l}^{2}\right]+\frac{3}{4}\left(x_{H}-x_{u}+x_{d}\right), \\
\Delta Z_{m_{d}}^{(1)} & =-\frac{1}{3}\left(\frac{1}{c_{\theta}^{2}}+1-2 c_{\theta}^{2}\right)+\frac{3}{x_{H}}\left[\frac{1}{2} \frac{1}{c_{\theta}^{4}}+1-2\left(X_{u}^{2}+X_{d}^{2}\right)-\frac{2}{3} X_{l}^{2}\right]+\frac{3}{4}\left(x_{H}+x_{u}-x_{d}\right) .
\end{aligned}
$$

\section{Two-loop result}

Before showing explicit expressions for the individual components of Eq.(134) let us recall that scaled masses are defined by $x_{i}=M_{i}^{2} / M^{2}$, where $M$ is the renormalized $W$-boson mass, and where $y_{i}=M_{i}^{2} / s$, with $s=-p^{2} ; p$ is the external momentum. Each term in the r.h.s. of Eq.(134) develops a non-local residue containing the (logarithmic) function

$$
L_{\beta}(x)=\ln \frac{\beta(x)+1}{\beta(x)-1},
$$

where $\beta$ can be found in Eq. (21). We will show explicitly that non-local residues cancel when summing up all the contributions, and UV poles will be subtracted by polynomial counterterms.

$\underline{\text { Two light-quark doublets }}$

$$
G_{d ; \operatorname{irr} ; U V ; 1}^{A A ; 2 ; l q}(s)=2 G_{d ; \text { irr;UV;3 }}^{A A ; 2 ; l q}(s)=-\frac{g^{4} s_{\theta}^{2}}{\pi^{4}} \frac{3}{64} s,
$$




$$
G_{d ; \text { irr } ; U V ; 2}^{A A ; l q}(s)=\frac{g^{4} s_{\theta}^{2}}{\pi^{4}} \frac{1}{8}\left\{\frac{s}{108}\left(\frac{115}{8}+\frac{73}{8} \frac{1}{c_{\theta}^{2}}+17 c_{\theta}^{2}\right)+\frac{3}{2} M^{2}+\frac{L_{\beta}\left(y_{W}\right)}{\beta\left(y_{W}\right)}\left[-\frac{s}{8}+M^{2}\left(\frac{1}{4}+3 y_{W}\right)\right]\right\} .
$$

$\underline{\text { Top-bottom doublet }}$

$$
\begin{aligned}
& G_{d ; \text { irr } ; U V ; 1}^{A A ; 2 ; t b}(s)=2 G_{d ; \text { irr } ; U V ; 3}^{A A ; 2 ; t b}(s)=-\frac{g^{4} s_{\theta}^{2}}{\pi^{4}} \frac{3}{128} s \\
& G_{d ; \text { irr } ; U V ; 2}^{A A ; 2 ; t b}(s)= \frac{g^{4} s_{\theta}^{2}}{\pi^{4}}\left\{\frac{s}{64}\left[\frac{1}{27}\left(\frac{73}{8} \frac{1}{c_{\theta}^{2}}+c_{\theta}^{2}\right)+\frac{1}{8}\left(9-\frac{13}{3} x_{t}\right)\right]\right. \\
&+ \frac{3}{32} M^{2}\left(1-\frac{3}{2} x_{t}-x_{t}^{2}\right)+\frac{1}{16} \frac{L_{\beta}\left(y_{W}\right)}{\beta\left(y_{W}\right)}\left[-\frac{s}{8}+\frac{M^{2}}{4}\left(1-\frac{9}{2} x_{t}+3 x_{t}^{2}\right)\right. \\
&+\left.\left.3 M^{2} y_{W}\left(1-\frac{3}{2} x_{t}-\frac{3}{4} x_{t}^{2}\right)\right]+\frac{s}{36} \frac{g_{S}^{2}}{g^{2}}\right\} .
\end{aligned}
$$

Note the presence of mixed electroweak-QCD corrections proportional to the strong coupling constant $g_{S}$, which do not show up in Eq.(139) because they are subtracted from the light-quark components.

Three lepton doublets

$$
\begin{gathered}
G_{d ; \text { irr } ; U V ; 1}^{A A ; 2 \text { lep }}(s)=2 G_{d ; \text { ir } ; U V ; 3}^{A A ; 2 ; \text { lep }}(s)=-\frac{g^{4} s_{\theta}^{2}}{\pi^{4}} \frac{3}{128} s \\
G_{d ; \text { irr;UV;2 }}^{A A ; \text { lep }}(s)=\frac{g^{4} s_{\theta}^{2}}{\pi^{4}}\left\{\frac{s}{512}\left(1+15 \frac{1}{c_{\theta}^{2}}\right)+\frac{3}{32} M^{2}+\frac{1}{16} \frac{L_{\beta}\left(y_{W}\right)}{\beta\left(y_{W}\right)}\left[-\frac{s}{8}+M^{2}\left(\frac{1}{4}+3 y_{W}\right)\right]\right\} .
\end{gathered}
$$

$\underline{\text { Bosonic contributions }}$

$$
\begin{gathered}
G_{d ; \text { irr } ; U V ; 1}^{A A ; 2 ; \text { bos }}(s)=\frac{g^{4} s_{\theta}^{2}}{\pi^{4}} \frac{47}{768} s \\
G_{d ; \text { irr; } ; \text {; } ; 2}^{A A ; 2}(s)=\frac{g^{4} s_{\theta}^{2}}{\pi^{4}}\left\{\frac{s}{256}\left(-\frac{21}{4}+\frac{1}{c_{\theta}^{2}}\right)+\frac{3}{8} M^{2}\left(-1+\frac{3}{8} x_{t}+\frac{1}{4} x_{t}^{2}\right)\right. \\
\left.+\frac{1}{4} \frac{L_{\beta}\left(y_{W}\right)}{\beta\left(y_{W}\right)}\left[\frac{s}{8}+\frac{M^{2}}{2}\left(-\frac{1}{2}+\frac{9}{16} x_{t}-\frac{3}{8} x_{t}^{2}\right)+M^{2} y_{W}\left(-3+\frac{9}{8} x_{t}+\frac{9}{16} x_{t}^{2}\right)\right]\right\} \\
G_{d ; \text { irr } ; U V ; 3}^{A A ; 2 ; \text { bos }}(s)=\frac{g^{4} s_{\theta}^{2}}{\pi^{4}} \frac{33}{1024} s .
\end{gathered}
$$

$\underline{\text { Total contributions }}$

The sum of the four components gives

$$
\begin{gathered}
G_{d ; \text { irr } ; U V ; 1}^{A A ; 2}(s)=-\frac{g^{4} s_{\theta}^{2}}{\pi^{4}} \frac{25}{768} s, \\
G_{d ; \text { irr } ; U V ; 2}^{A A ;}(s)=\frac{g^{4} s_{\theta}^{2}}{\pi^{4}} \frac{s}{192}\left(\frac{433}{144}+\frac{113}{12} \frac{1}{c_{\theta}^{2}}+\frac{35}{9} c_{\theta}^{2}-\frac{13}{8} x_{t}+\frac{16}{3} \frac{g_{S}^{2}}{g^{2}}\right), \\
G_{d ; \text { irr } ; U V ; 3}^{A A ; 2}(s)=-\frac{g^{4} s_{\theta}^{2}}{\pi^{4}} \frac{15}{1024} s .
\end{gathered}
$$

Non-local residues cancel and the UV residue can be reabsorbed by the counterterm contributions,

$$
G_{d ; \text { irr } ; \text { it } ; a}^{A A ;}(s)=\frac{g^{4} s}{256 \pi^{4}} \Delta Z_{A A ; a}^{(2)}, \quad a=1,2,3 .
$$

\section{An alternative method}



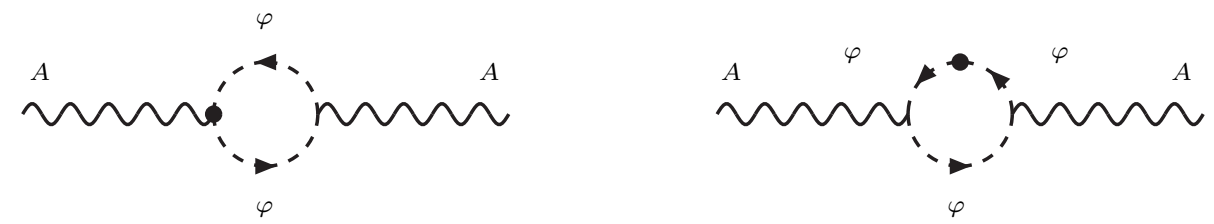

Figure 12: Counterterm-dependent Feynman diagrams with a $\varphi^{ \pm}$loop. Dotted vertices represent counterterm insertions.

Here we outline another strategy to verify the cancellation of logarithmic residues. Let us consider Fig. 12 , corresponding to two Feynman diagrams with a Higgs-Kibble $\varphi^{ \pm}$loop and a one-loop counterterm insertion. The contributions of these two diagrams is given by

$$
\begin{gathered}
H_{\mu \nu ; 1}^{A A ; 2}=\frac{g^{4} s_{\theta}^{2}}{16 \pi^{2}} \mu^{4-n} \int d^{n} q \frac{(2 q+p)_{\mu}(2 q+p)_{\nu}}{\left(q^{2}+M^{2}\right)\left[(q+p)^{2}+M^{2}\right]}\left(\delta Z_{g}^{(1)}+\delta Z_{s_{\theta}}^{(1)}+\delta Z_{\varphi}^{(1)}+\frac{\delta Z_{A A}^{(1)}}{2}\right) \\
H_{\mu \nu ; 2}^{A A ; 2}=-\frac{g^{4} s_{\theta}^{2}}{16 \pi^{2}} \mu^{4-n} \int d^{n} q \frac{(2 q+p)_{\mu}(2 q+p)_{\nu}}{\left(q^{2}+M^{2}\right)^{2}\left[(q+p)^{2}+M^{2}\right]}\left[\left(q^{2}+M^{2}\right) \delta Z_{\varphi}^{(1)}+M^{2}\left(\delta Z_{M}^{(1)}+2 \delta Z_{\xi_{\varphi}}^{(1)}\right)\right]
\end{gathered}
$$

After summing Eqs.151)-152) the terms which depend on $\delta Z_{\varphi}^{(1)}$ cancel out,

$$
\begin{aligned}
H_{\mu \nu ; 1}^{A A ; 2} & +H_{\mu \nu ; 2}^{A A ; 2}=\frac{g^{4} s_{\theta}^{2}}{16 \pi^{2}} \mu^{4-n} \int d^{n} q \frac{(2 q+p)_{\mu}(2 q+p)_{\nu}}{\left(q^{2}+M^{2}\right)\left[(q+p)^{2}+M^{2}\right]} \\
& \times\left[\delta Z_{g}^{(1)}+\delta Z_{s_{\theta}}^{(1)}+\frac{\delta Z_{A A}^{(1)}}{2}-\frac{M^{2}}{q^{2}+M^{2}}\left(\delta Z_{M}^{(1)}+2 \delta Z_{\xi_{\varphi}}^{(1)}\right)\right] .
\end{aligned}
$$

Moreover, after using the relation

$$
\delta Z_{g}^{(1)}=-\delta Z_{s_{\theta}}^{(1)}-\frac{1}{2} \delta Z_{A A}^{(1)}
$$

the answer contains only the $W$-mass- and gauge-parameter-dependent counterterms,

$$
H_{\mu \nu ; 1}^{A A ; 2}+H_{\mu \nu ; 2}^{A A ; 2}=-\frac{g^{4} s_{\theta}^{2} M^{2}}{16 \pi^{2}}\left(\delta Z_{M}^{(1)}+2 \delta Z_{\xi_{\varphi}}^{(1)}\right) \mu^{4-n} \int d^{n} q \frac{(2 q+p)_{\mu}(2 q+p)_{\nu}}{\left(q^{2}+M^{2}\right)^{2}\left[(q+p)^{2}+M^{2}\right]} .
$$

Furthermore, all Feynman diagrams with the insertion of counterterms can be paired like in Fig. 12 and we can repeat the argument for any of the pairs. Consequently, counterterms for masses and gauge parameters suffice in removing logarithmic residues. Here we outline the procedure: we take in account the irreducible Green function at $\mathcal{O}\left(g^{2}\right)$ which are written as follows:

$$
G_{d ; \text { irr }}^{A A ; 1}(s)=G_{d ; \text { irr }}^{A A ; 1 \text { fer }}(s)+G_{d ; \text { irr }}^{A A ; 1 ; \text { bos }}(s)
$$

where the first term receives contributions from the three lepton families and the top quark (light-quark components are subtracted), whereas the second one gets contributions from bosonic loops. The essential point is that only bare quantities appear in Eq.(156); in other words, the gauge-fixing terms are

$$
\mathcal{C}^{A}=-\frac{1}{\xi_{A}^{b}} \partial_{\mu} A_{\mu}^{b}-\xi_{A Z}^{b} \partial_{\mu} Z_{\mu}^{b}, \quad \mathcal{C}^{Z}=-\frac{1}{\xi_{Z}^{b}} \partial_{\mu} Z_{\mu}^{b}+\xi_{\varphi^{0}}^{b} M_{0}^{b} \varphi^{0, b}, \quad \mathcal{C}^{ \pm}=-\frac{1}{\xi_{W}^{b}} \partial_{\mu} W_{\mu}^{ \pm, b}+\xi_{\varphi^{b}}^{b} M^{b} \varphi^{ \pm, b}
$$


where we used the superscript $b$ to denote bare quantities. Next, we use fermion mass renormalization, $m^{b}=Z_{m}^{1 / 2} m$, where the renormalization constant is expanded as

$$
Z_{m}=1+\frac{g^{2}}{16 \pi^{2}} \delta Z_{m}^{(1)}+\mathcal{O}\left(g^{4}\right)
$$

and the counterterms for lepton and top masses can be read in Eq.91). We rewrite the first term in the r.h.s. of Eq.(156) as

$$
G_{d ; \text { irr }}^{A A ; 1 \text { fer }}(s)=G_{d ; \text { irr } ; \text { ren }}^{A A ; 1 \text { fer }}(s)+\Delta G_{d ; \text { irr } ; \text { ren }}^{A A ; 2 ; \text { fer }}(s),
$$

where functions in in the r.h.s. of Eq.(159) depend on renormalized quantities. The first term in r.h.s. of Eq.(159) represents the one-loop fermionic contribution to the renormalized photon self-energy, whereas the second term gives an additional contribution to the two-loop result.

We verified that, after adding the second term in the r.h.s. of Eq.(159) to two-loop diagrams, the answer is free from logarithmic residues. Therefore, fermion-mass renormalization removes all logarithms in the residue of the simple UV pole for the fermionic part.

However, a non-local residue remains in the bosonic part. Unfortunately, a simple procedure of $W$-mass renormalization is not enough to get rid of logarithmic residues in the bosonic component and the reason is that in a bosonic loop we may have three different fields, the $W$, the $\varphi$ and the charged ghost fields, and only one mass is available. The situation is illustrated in Fig. 13 where the black dot denotes insertion of a counterterm $\delta Z_{M}$. The latter is fixed in order to remove the UV pole in the $W$ self-energy and one verifies that the total in the second and third line of Fig. 13 ( $\varphi$ and $X$ self-energies, respectively) is not UV finite. The procedure has to be changed if we want to make the result in the bosonic sector as similar as possible
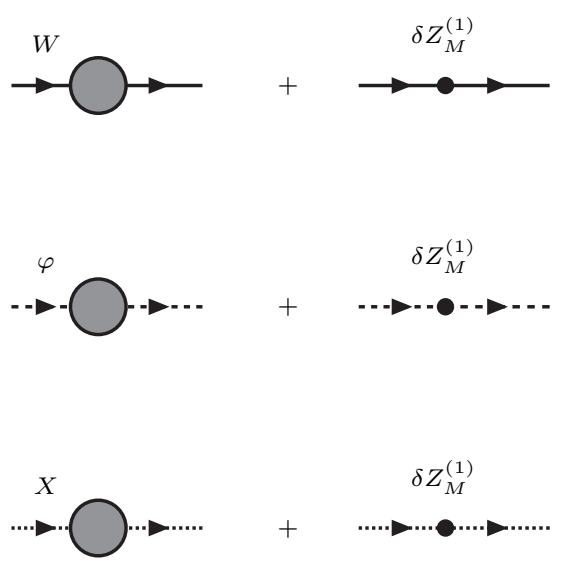

Figure 13: $W$-mass counterterm insertion in the charged one-loop transitions. The $W-W$ one is UV finite, whereas the same is not true for $\varphi-\varphi$ and ghost-ghost transitions.

to the one in the fermionic sector. A diagrammatic interpretation of the method is displayed in Fig. 15, For the bosonic part we have to take into account Dyson-resummed propagators. Let us consider the following integral, corresponding to a $\varphi$ loop in the photon self-energy,

$$
H_{\mu \nu}^{A A ; 1}=\left(g^{b}\right)^{2}\left(s_{\theta}^{b}\right)^{2} \mu^{4-n} \int d^{n} q \frac{\left[q^{2}+\left(\xi_{W}^{b}\right)^{2} M_{b}^{2}\right]\left[(q+p)^{2}+\left(\xi_{W}^{b}\right)^{2} M_{b}^{2}\right]}{\left[q^{2}+\xi_{W}^{b} \xi_{\varphi}^{b} M_{b}^{2}\right]\left[(q+p)^{2}+\xi_{W}^{b} \xi_{\varphi}^{b} M_{b}^{2}\right]}\left(2 q_{\mu}+p_{\mu}\right)\left(2 q_{\nu}+p_{\nu}\right) .
$$

We introduce renormalized quantities for the gauge parameters and the $W$-boson mass,

$$
M^{b}=Z_{M}^{1 / 2} M, \quad \xi_{W}=Z_{\xi_{W}} \xi_{W}, \quad \xi_{\varphi}=Z_{\xi_{\varphi}} \xi_{\varphi},
$$


and expand the renormalization constants,

$$
Z_{i}=1+\frac{g^{2}}{16 \pi^{2}} \delta Z_{i}^{(1)}+\mathcal{O}\left(g^{2}\right)
$$

For the propagators we get

$$
\left[q^{2}+\xi_{\varphi}^{b} \xi_{W}^{b} M_{b}^{2}\right]^{-1}=\left(q^{2}+M^{2}\right)^{-1}-\frac{g^{2}}{16 \pi^{2}}\left(\delta Z_{\xi_{W}}+\delta Z_{\xi_{\varphi}}+\delta Z_{M}\right) M^{2}\left(q^{2}+M^{2}\right)^{-2}+\ldots
$$

Therefore, each one-loop bosonic diagram can be written as

$$
H_{d ; \text { irr }}^{A A ; 1}(s)=H_{d ; \text { irr } ; \text { ren }}^{A A ;}(s)+\Delta H_{d ; \text { irr } ; \text { ren }}^{A A ; 2}(s)
$$

where the second term in the r.h.s. of Eq.(164) gives an additional contribution to the two-loop photon self-energy which depends on the $W$-mass and gauge-parameter counterterms. Collecting all the diagrams, we get

$$
G_{d ; \text { irr }}^{A A ; 1 ; \text { bos }}(s)=G_{d ; \text { irr } ; \text { ren }}^{A A ; 1 ; \text { bos }}(s)+\Delta G_{d ; \text { irr } ; \text { ren }}^{A A ;}(s) .
$$

We verified that $W$-mass and gauge-parameter renormalization removes all the non-local residues also for the bosonic component of the photon two-loop self-energy.

We emphasize the importance of using the $R_{\xi \xi}$ gauge of Eq.(58): renormalization of gauge parameters is essential in removing UV divergencies at one loop; furthermore, unitarity requires that any subtraction term must be local.

Summarizing, we have been able to verify that the electroweak theory can be made (two-loop) UV finite by adding local counterterms with two different methods, each leading to the same result. In other words, the well-known one-loop result that self-energies suffice in performing renormalization can be extended up to two loops. Although counterterms have been explicitly included it remains true that:

- In extracting $\alpha$ from Thomson scattering at zero momentum transfer we find four classes of two-loop diagrams:

I) irreducible two-loop vertices and wave-function factors, product of one-loop corrected vertices with one-loop wave-function factors;

II) one-loop vacuum polarization $\otimes$ one-loop vertices or one-loop wave-function factors;

III) irreducible two-loop $A A, A Z, A \phi^{0}$ transitions;

IV) reducible two-loop $A A, A Z, A \phi^{0}$ transitions.

We have verified that the non-vanishing contribution originates from III and IV only and, within these terms, only the reducible and irreducible $A A$ transition survives. For this result the role of $\Gamma$ is vital.

- In extracting the Fermi coupling constant from the muon lifetime all corrections to

$$
\frac{G_{F}}{\sqrt{2}}=\frac{g^{2}}{8 M^{2}}(1+\Delta g)
$$

which do not originate from the $W$ self-energy and that are UV (and IR) finite at one loop remain finite at two loops after one-loop renormalization (i.e. two-loop counterterms are not needed),

The proof has been obtained by using $\mathcal{G}$ raphShot [8], generating the whole set of corrections. The result follows by algebraic methods (see Appendix (D), i.e. full reduction of tensor structures without using the explicit expressions for the scalar integrals. 


\subsection{The $\gamma-Z$ transition}

For the transition between the photon and the $Z$ boson we show again all the various components of Eq.(134).

$\underline{\text { Two light-quark doublets }}$

$$
\begin{gathered}
G_{d ; \text { irr } ; U V ; 1}^{A Z ; 2 ; l q}(s)=2 G_{d ; \text { irr } ; U V ; 3}^{A Z ; 2 ; l q}(s)=\frac{g^{4}}{\pi^{4}} \frac{s_{\theta}}{c_{\theta}} \frac{3}{64}\left(-c_{\theta}^{2} s+\frac{M^{2}}{2}\right), \\
G_{d ; \text { irr } ; U V ; 2}^{A Z ; l q}(s)=\frac{g^{4}}{\pi^{4}} \frac{s_{\theta}}{c_{\theta}}\left\{\frac{s}{54}\left(1-\frac{137}{256} \frac{1}{c_{\theta}^{2}}+\frac{251}{128} c_{\theta}^{2}\right)+\frac{M^{2}}{16}\left(\frac{29}{32}+3 c_{\theta}^{2}\right)\right. \\
\left.+\frac{1}{8} \frac{L_{\beta}\left(y_{W}\right)}{\beta\left(y_{W}\right)}\left[-s \frac{c_{\theta}^{2}}{8}+M^{2}\left(-\frac{3}{16}+\frac{c_{\theta}^{2}}{4}\right)+M^{2} y_{W}\left(1+3 c_{\theta}^{2}\right)\right]+\frac{g_{S}^{2}}{g^{2}} s\left(\frac{5}{72} c_{\theta}^{2}-\frac{11}{288}\right)\right\} .
\end{gathered}
$$

$\underline{\text { Top-bottom doublet }}$

$$
\begin{aligned}
& G_{d ; \text { irr } ; U V ; 1}^{A Z ; 2 ; t b}(s)=2 G_{d ; \text { irr } ; U V ; 3}^{A Z ; 2 ; t b}(s)=\frac{g^{4}}{\pi^{4}} \frac{s_{\theta}}{c_{\theta}} \frac{3}{128}\left(-c_{\theta}^{2} s+\frac{M^{2}}{2}\right) \\
& G_{d ; \text { ir } ; U V ; 2}^{A Z ; 2 ; t b}(s)= \frac{g^{4}}{\pi^{4}} \frac{s_{\theta}}{c_{\theta}}\left\{\frac{s}{12}\left(\frac{1}{9}-\frac{137}{2304} \frac{1}{c_{\theta}^{2}}-\frac{13}{128} c_{\theta}^{2} x_{t}+\frac{251}{1152} c_{\theta}^{2}+\frac{17}{256} x_{t}\right)\right. \\
&+\frac{M^{2}}{32}\left(\frac{29}{32}-\frac{9}{2} c_{\theta}^{2} x_{t}-3 c_{\theta}^{2} x_{t}^{2}+3 c_{\theta}^{2}-\frac{3}{4} x_{t}+\frac{3}{2} x_{t}^{2}\right) \\
&+\frac{1}{16} \frac{L_{\beta}\left(y_{W}\right)}{\beta\left(y_{W}\right)}\left[-\frac{s}{8} c_{\theta}^{2}+\frac{M^{2}}{4}\left(-\frac{3}{4}-\frac{9}{2} c_{\theta}^{2} x_{t}+3 c_{\theta}^{2} x_{t}^{2}+c_{\theta}^{2}+\frac{9}{4} x_{t}-\frac{3}{2} x_{t}^{2}\right)\right. \\
&\left.\left.+M^{2} y_{W}\left(1-\frac{9}{2} c_{\theta}^{2} x_{t}-\frac{9}{4} c_{\theta}^{2} x_{t}^{2}+3 c_{\theta}^{2}-\frac{9}{8} x_{t}+\frac{3}{4} x_{t}^{2}\right)\right]+\frac{g_{S}^{2}}{g^{2}} s\left(\frac{5}{144} c_{\theta}^{2}-\frac{11}{576}\right)\right\}
\end{aligned}
$$

$\underline{\text { Three lepton doublets }}$

$$
\begin{gathered}
G_{d ; \text { irr } ; U V ; 1}^{A Z ; 2 ; \text { lep }}(s)=2 G_{d ; \text { irr } ; U V ; 3}^{A Z ; \text { lep }}(s)=\frac{g^{4}}{\pi^{4}} \frac{s_{\theta}}{c_{\theta}} \frac{3}{128}\left(-c_{\theta}^{2} s+\frac{M^{2}}{2}\right), \\
G_{d ; \text { irr } ; U V ; 2}^{A Z ; 2 ; \text { lep }}(s)=\frac{g^{4}}{\pi^{4}} \frac{s_{\theta}}{c_{\theta}}\left\{\frac{s}{64}\left(3-\frac{27}{16} \frac{1}{c_{\theta}^{2}}+\frac{1}{8} c_{\theta}^{2}\right)+\frac{M^{2}}{32}\left(\frac{29}{32}+3 c_{\theta}^{2}\right)\right. \\
\left.+\frac{1}{16} \frac{L_{\beta}\left(y_{W}\right)}{\beta\left(y_{W}\right)}\left[-\frac{s}{8} c_{\theta}^{2}+\frac{M^{2}}{4}\left(-\frac{3}{4}+c_{\theta}^{2}\right)+M^{2} y_{W}\left(1+3 c_{\theta}^{2}\right)\right]\right\} .
\end{gathered}
$$

$\underline{\text { Bosonic contributions }}$

$$
\begin{aligned}
& G_{d ; \text { irr } ; U V ; 1}^{A Z ; 2}(s)=\frac{g^{4}}{\pi^{4}} \frac{s_{\theta}}{c_{\theta}} \frac{1}{64}\left[\frac{s}{2}\left(-\frac{943}{18}+119 c_{\theta}^{2}-\frac{529}{9} c_{\theta}^{4}\right)-3 M^{2}\right], \\
& G_{d ; \text { irr } ; U V ; 2}^{A Z ; 2 ; \text { bos }}(s)= \frac{g^{4}}{\pi^{4}} \frac{s_{\theta}}{c_{\theta}}\left\{\frac{s}{512}\left(-\frac{1}{c_{\theta}^{2}}-\frac{21}{2} c_{\theta}^{2}\right)+\frac{M^{2}}{8}\left(-\frac{29}{32}+\frac{9}{8} c_{\theta}^{2} x_{t}+\frac{3}{4} c_{\theta}^{2} x_{t}^{2}-3 c_{\theta}^{2}+\frac{3}{16} x_{t}-\frac{3}{8} x_{t}^{2}\right)\right. \\
&+\frac{1}{4} \frac{L_{\beta}\left(y_{W}\right)}{\beta\left(y_{W}\right)}\left[\frac{s}{8} c_{\theta}^{2}+\frac{M^{2}}{4}\left(\frac{3}{4}+\frac{9}{8} c_{\theta}^{2} x_{t}-\frac{3}{4} c_{\theta}^{2} x_{t}^{2}-c_{\theta}^{2}-\frac{9}{16} x_{t}+\frac{3}{8} x_{t}^{2}\right)\right. \\
&\left.\left.+M^{2} y_{W}\left(-1+\frac{9}{8} c_{\theta}^{2} x_{t}+\frac{9}{16} c_{\theta}^{2} x_{t}^{2}-3 c_{\theta}^{2}+\frac{9}{32} x_{t}-\frac{3}{16} x_{t}^{2}\right)\right]\right\},
\end{aligned}
$$




$$
G_{d ; \text { irr } ; U V ; 3}^{A Z ; 2 ; \text { bos }}(s)=\frac{g^{4}}{\pi^{4}} \frac{s_{\theta}}{c_{\theta}} \frac{1}{128}\left[\frac{s}{12}\left(-\frac{1886}{3}+383 c_{\theta}^{2}-\frac{529}{3} c_{\theta}^{4}\right)-3 M^{2}\right] .
$$

\section{$\underline{\text { Total contributions }}$}

The sum of the four components gives

$$
\begin{gathered}
G_{d ; \text { irr } ; U V ; 1}^{A Z ; 2}(s)=\frac{g^{4}}{\pi^{4}} \frac{s_{\theta}}{c_{\theta}} \frac{s}{128}\left(-\frac{943}{18}+107 c_{\theta}^{2}-\frac{529}{9} c_{\theta}^{4}\right), \\
G_{d ; \text { irr } ; U V ; 2}^{A Z ; 2}(s)=\frac{g^{4}}{\pi^{4}} \frac{s_{\theta}}{c_{\theta}} \frac{s}{64}\left[\frac{43}{9}-\frac{199}{72} \frac{1}{c_{\theta}^{2}}-\frac{13}{24} c_{\theta}^{2} x_{t}+\frac{331}{144} c_{\theta}^{2}+\frac{17}{48} x_{t}+\frac{g_{S}^{2}}{g^{2}}\left(\frac{20}{3} c_{\theta}^{2}-\frac{11}{3}\right)\right], \\
G_{d ; \text { irr } ; U V ; 3}^{A Z ; 2}(s)=\frac{g^{4}}{\pi^{4}} \frac{s_{\theta}}{c_{\theta}} \frac{s}{1536}\left(-\frac{1886}{3}+311 c_{\theta}^{2}-\frac{529}{3} c_{\theta}^{4}\right),
\end{gathered}
$$

and can be absorbed by the contribution of the counterterms,

$$
G_{d ; \text { irr } ; U V ; a}^{A Z ; 2}(s)=\frac{g^{4} s}{256 \pi^{4}} \Delta Z_{A Z ; a}^{(2)}, \quad a=1,2,3 .
$$

\subsection{The $Z$-boson self-energy}

The explicit expressions for the individual components of the $Z$-boson self-energy are rather lengthy. Here we provide the total result for the coefficients of the UV factors and we show that non-local residues cancel,

$$
\begin{aligned}
G_{d ; \text { irr } ; U V ; 1}^{Z Z ; 2}(s) & =\frac{g^{4}}{\pi^{4} c_{\theta}^{2}}\left[\frac{s}{576}\left(\frac{1681}{4}-\frac{5453}{4} c_{\theta}^{2}+\frac{5813}{4} c_{\theta}^{4}-529 c_{\theta}^{6}\right)\right. \\
& +\frac{M^{2}}{32}\left(\frac{10385}{288}+\frac{9}{8} \frac{1}{c_{\theta}^{8} x_{H}^{2}}-\frac{25}{4} \frac{1}{c_{\theta}^{6} x_{H}}-9 \frac{x_{t}^{2}}{c_{\theta}^{4} x_{H}^{2}}+\frac{9}{2} \frac{1}{c_{\theta}^{4} x_{H}^{2}}+\frac{9}{4} \frac{x_{t}}{c_{\theta}^{4} x_{H}}\right. \\
& +\frac{59}{8} \frac{1}{c_{\theta}^{4} x_{H}}+\frac{4667}{576} \frac{1}{c_{\theta}^{4}}+\frac{1}{4} \frac{x_{t}^{2}}{c_{\theta}^{2} x_{H}}-5 \frac{1}{c_{\theta}^{2} x_{H}}-\frac{1}{32} \frac{x_{t}}{c_{\theta}^{2}}-\frac{425}{18} \frac{1}{c_{\theta}^{2}} \\
& -\frac{1771}{72} c_{\theta}^{2}+\frac{529}{72} c_{\theta}^{4}-18 \frac{x_{t}^{2}}{x_{H}^{2}}+18 \frac{x_{t}^{4}}{x_{H}^{2}}+\frac{9}{2} \frac{1}{x_{H}^{2}}+\frac{9}{2} \frac{x_{t}}{x_{H}}+9 \frac{x_{t}^{2}}{x_{H}}-\frac{9}{4} \frac{x_{t}^{3}}{x_{H}} \\
& \left.\left.-\frac{1}{x_{H}}-\frac{5}{16} x_{H}-\frac{9}{64} x_{H}^{2}-\frac{23}{16} x_{t}-\frac{117}{32} x_{t}^{2}\right)+\frac{g_{S}^{2}}{g^{2}} M^{2}\left(-\frac{3}{4} \frac{x_{t}^{2}}{x_{H}}+\frac{3}{32} x_{t}\right)\right], \\
G_{d ; \text { irr } ; U V ; 2}^{Z Z ; 2}= & \frac{g^{4}}{\pi^{4} c_{\theta}^{2}}\left[\frac{s}{384}\left(-43+\frac{199}{12} \frac{1}{c_{\theta}^{2}}+\frac{17}{4} c_{\theta}^{2} x_{t}+\frac{77}{2} c_{\theta}^{2}-\frac{13}{4} c_{\theta}^{4} x_{t}+\frac{331}{24} c_{\theta}^{4}-\frac{17}{8} x_{t}\right)\right. \\
+ & \frac{M^{2}}{32}\left(-\frac{5}{12}+\frac{379}{96} \frac{1}{c_{\theta}^{6} x_{H}}+\frac{19}{16} \frac{x_{t}}{c_{\theta}^{4} x_{H}}-\frac{289}{48} \frac{1}{c_{\theta}^{4} x_{H}}-\frac{3383}{2304} \frac{1}{c_{\theta}^{4}}-5 \frac{x_{t}}{c_{\theta}^{2} x_{H}}\right. \\
+ & \frac{1}{3} \frac{x_{t}^{2}}{c_{\theta}^{2} x_{H}}+\frac{77}{24} \frac{1}{c_{\theta}^{2} x_{H}}-\frac{3}{32} \frac{x_{H}}{c_{\theta}^{2}}-\frac{85}{384} \frac{x_{t}}{c_{\theta}^{2}}+\frac{4}{3} \frac{1}{c_{\theta}^{2}}+\frac{35}{8} \frac{x_{t}}{x_{H}}-\frac{1}{3} \frac{x_{t}^{2}}{x_{H}} \\
& \left.-\frac{15}{8} \frac{x_{t}^{3}}{x_{H}}-\frac{32}{3} \frac{1}{x_{H}}+\frac{9}{32} x_{t} x_{H}-\frac{3}{16} x_{H}+\frac{63}{256} x_{H}^{2}-\frac{25}{192} x_{t}-\frac{21}{128} x_{t}^{2}\right) \\
+ & \left.\frac{g_{S}^{2}}{g^{2}} s\left(\frac{11}{192}-\frac{11}{96} c_{\theta}^{2}+\frac{5}{48} c_{\theta}^{4}\right)+\frac{g_{S}^{2}}{g^{2}} M^{2}\left(\frac{1}{8} \frac{x_{t}^{2}}{x_{H}}-\frac{5}{128} x_{t}\right)\right], \\
G_{d ; \text { irr } ; U V ; 3}^{Z Z}(s)= & \frac{g^{4}}{\pi^{4} c_{\theta}^{2}}\left[\frac{s}{2304}\left(\frac{1681}{4}-\frac{5453}{4} c_{\theta}^{2}+\frac{5753}{4} c_{\theta}^{4}-529 c_{\theta}^{6}\right)\right. \\
+ & \frac{9737}{288}+\frac{9}{8} \frac{1}{c_{\theta}^{8} x_{H}^{2}}-\frac{25}{4} \frac{1}{c_{\theta}^{6} x_{H}}-9 \frac{x_{t}^{2}}{c_{\theta}^{4} x_{H}^{2}}+\frac{9}{2} \frac{1}{c_{\theta}^{4} x_{H}^{2}}+\frac{9}{4} \frac{x_{t}}{c_{\theta}^{4} x_{H}} \\
&
\end{aligned}
$$




$$
\begin{aligned}
& +\frac{59}{8} \frac{1}{c_{\theta}^{4} x_{H}}+\frac{4667}{576} \frac{1}{c_{\theta}^{4}}+\frac{1}{4} \frac{x_{t}^{2}}{c_{\theta}^{2} x_{H}}-2 \frac{1}{c_{\theta}^{2} x_{H}}-\frac{1}{32} \frac{x_{t}}{c_{\theta}^{2}}-\frac{425}{18} \frac{1}{c_{\theta}^{2}} \\
& -\frac{1771}{72} c_{\theta}^{2}+\frac{529}{72} c_{\theta}^{4}-18 \frac{x_{t}^{2}}{x_{H}^{2}}+18 \frac{x_{t}^{4}}{x_{H}^{2}}+\frac{9}{2} \frac{1}{x_{H}^{2}}+\frac{9}{2} \frac{x_{t}}{x_{H}}+9 \frac{x_{t}^{2}}{x_{H}}-\frac{9}{4} \frac{x_{t}^{3}}{x_{H}} \\
& \left.\left.+5 \frac{1}{x_{H}}-\frac{5}{16} x_{H}-\frac{9}{64} x_{H}^{2}-\frac{23}{16} x_{t}-\frac{117}{32} x_{t}^{2}\right)+\frac{g_{S}^{2}}{g^{2}} M^{2}\left(-\frac{3}{16} \frac{x_{t}^{2}}{x_{H}}+\frac{3}{128} x_{t}\right)\right] .
\end{aligned}
$$

As evident, the two-loop counterterms contain only local residues,

$$
G_{d ; \text { irr } ; U V ; a}^{Z Z ; 2 ; \mathrm{ct}}(s)=\frac{g^{4}}{256 \pi^{4}}\left[s \Delta Z_{Z ; a}^{(2)}-\frac{M^{2}}{c_{\theta}^{2}}\left(\Delta Z_{Z ; a}^{(2)}+\Delta Z_{M ; a}^{(2)}-2 \Delta Z_{c_{\theta} ; a}^{(2)}\right)\right] .
$$

\subsection{The $W$-boson self-energy}

For the $W$-boson self-energy we provide the total result which shows once again absence of non-local residues.

$$
\begin{aligned}
& G_{d ; i r r ; U V ; 1}^{W W ; 2}(s)=\frac{g^{4}}{\pi^{4}}\left[-\frac{25}{768} s+\frac{M^{2}}{32}\left(\frac{49}{18}+\frac{9}{8} \frac{1}{c_{\theta}^{8} x_{H}^{2}}-\frac{25}{4} \frac{1}{c_{\theta}^{6} x_{H}}\right.\right. \\
& -9 \frac{x_{t}^{2}}{c_{\theta}^{4} x_{H}^{2}}+\frac{9}{2} \frac{1}{c_{\theta}^{4} x_{H}^{2}}+\frac{9}{4} \frac{x_{t}}{c_{\theta}^{4} x_{H}}+\frac{59}{8} \frac{1}{c_{\theta}^{4} x_{H}}+\frac{145}{64} \frac{1}{c_{\theta}^{4}}+\frac{1}{4} \frac{x_{t}^{2}}{c_{\theta}^{2} x_{H}} \\
& -5 \frac{1}{c_{\theta}^{2} x_{H}}-\frac{1}{32} \frac{x_{t}}{c_{\theta}^{2}}-\frac{27}{16} \frac{1}{c_{\theta}^{2}}-18 \frac{x_{t}^{2}}{x_{H}^{2}}+18 \frac{x_{t}^{4}}{x_{H}^{2}}+\frac{9}{2} \frac{1}{x_{H}^{2}}+\frac{9}{2} \frac{x_{t}}{x_{H}} \\
& \left.\left.+9 \frac{x_{t}^{2}}{x_{H}}-\frac{9}{4} \frac{x_{t}^{3}}{x_{H}}-\frac{1}{x_{H}}-\frac{5}{16} x_{H}-\frac{9}{64} x_{H}^{2}-\frac{23}{16} x_{t}-\frac{117}{32} x_{t}^{2}\right)+\frac{g_{S}^{2}}{g^{2}} M^{2}\left(-\frac{3}{4} \frac{x_{t}^{2}}{x_{H}}+\frac{3}{32} x_{t}\right)\right] \\
& G_{d ; \text { irr } ; U V ; 2}^{W W ; 2}(s)=\frac{g^{4}}{\pi^{4}}\left[\frac{s}{512}\left(\frac{63}{2}+3 \frac{1}{c_{\theta}^{2}}-\frac{3}{2} x_{t}\right)\right. \\
& +\frac{M^{2}}{32}\left(-\frac{5}{12}+\frac{379}{96} \frac{1}{c_{\theta}^{6} x_{H}}+\frac{19}{16} \frac{x_{t}}{c_{\theta}^{4} x_{H}}-\frac{289}{48} \frac{1}{c_{\theta}^{4} x_{H}}-\frac{701}{768} \frac{1}{c_{\theta}^{4}}\right. \\
& -5 \frac{x_{t}}{c_{\theta}^{2} x_{H}}+\frac{1}{3} \frac{x_{t}^{2}}{c_{\theta}^{2} x_{H}}+\frac{77}{24} \frac{1}{c_{\theta}^{2} x_{H}}-\frac{3}{32} \frac{x_{H}}{c_{\theta}^{2}}-\frac{85}{384} \frac{x_{t}}{c_{\theta}^{2}}+\frac{4}{3} \frac{1}{c_{\theta}^{2}}+\frac{35}{8} \frac{x_{t}}{x_{H}} \\
& \left.-\frac{1}{3} \frac{x_{t}^{2}}{x_{H}}-\frac{15}{8} \frac{x_{t}^{3}}{x_{H}}-\frac{32}{3} \frac{1}{x_{H}}+\frac{9}{32} x_{t} x_{H}-\frac{3}{16} x_{H}+\frac{63}{256} x_{H}^{2}-\frac{25}{192} x_{t}-\frac{21}{128} x_{t}^{2}\right) \\
& \left.+\frac{g_{S}^{2}}{g^{2}} M^{2}\left(\frac{1}{8} \frac{x_{t}^{2}}{x_{H}}-\frac{5}{128} x_{t}\right)+\frac{g_{S}^{2}}{g^{2}} s \frac{3}{64}\right] \\
& G_{d ; \mathrm{irr} ; U V ; 3}^{W W ; 2}(s)=\frac{g^{4}}{\pi^{4}}\left[-\frac{15}{1024} s+\frac{M^{2}}{32}\left(\frac{17}{144}+\frac{9}{32} \frac{1}{c_{\theta}^{8} x_{H}^{2}}-\frac{25}{16} \frac{1}{c_{\theta}^{6} x_{H}}\right.\right. \\
& -\frac{9}{4} \frac{x_{t}^{2}}{c_{\theta}^{4} x_{H}^{2}}+\frac{9}{8} \frac{1}{c_{\theta}^{4} x_{H}^{2}}+\frac{9}{16} \frac{x_{t}}{c_{\theta}^{4} x_{H}}+\frac{59}{32} \frac{1}{c_{\theta}^{4} x_{H}}+\frac{145}{256} \frac{1}{c_{\theta}^{4}}+\frac{1}{16} \frac{x_{t}^{2}}{c_{\theta}^{2} x_{H}} \\
& -\frac{1}{2} \frac{1}{c_{\theta}^{2} x_{H}}-\frac{1}{128} \frac{x_{t}}{c_{\theta}^{2}}-\frac{27}{64} \frac{1}{c_{\theta}^{2}}-\frac{9}{2} \frac{x_{t}^{2}}{x_{H}^{2}}+\frac{9}{2} \frac{x_{t}^{4}}{x_{H}^{2}}+\frac{9}{8} \frac{1}{x_{H}^{2}}+\frac{9}{8} \frac{x_{t}}{x_{H}} \\
& \left.+\frac{9}{4} \frac{x_{t}^{2}}{x_{H}}-\frac{9}{16} \frac{x_{t}^{3}}{x_{H}}+\frac{5}{4} \frac{1}{x_{H}}-\frac{5}{64} x_{H}-\frac{9}{256} x_{H}^{2}-\frac{23}{64} x_{t}-\frac{117}{128} x_{t}^{2}\right) \\
& \left.+\frac{g_{S}^{2}}{g^{2}} M^{2}\left(-\frac{3}{16} \frac{x_{t}^{2}}{x_{H}}+\frac{3}{128} x_{t}\right)\right] \text {. } \\
& G_{d ; \text { irr;UV;a }}^{W W ; 2 ; \mathrm{ct}}(s)=\frac{g^{4}}{256 \pi^{4}}\left[s \Delta Z_{W ; a}^{(2)}-M^{2}\left(\Delta Z_{W ; a}^{(2)}+\Delta Z_{M ; a}^{(2)}\right)\right] .
\end{aligned}
$$




\subsection{The Higgs-boson self-energy}

Here we provide the result for the Higgs-boson self-energy, showing the absence of non-local residues,

$$
\begin{aligned}
& G_{U V ; \text { irr } ; 1}^{H H}(s)=\frac{g^{4}}{\pi^{4}}\left[\frac{s}{64}\left(-1-\frac{43}{16} \frac{1}{c_{\theta}^{4}}-\frac{5}{16} \frac{x_{t}}{c_{\theta}^{2}}+\frac{37}{8} \frac{1}{c_{\theta}^{2}}+\frac{7}{8} x_{t}+\frac{9}{16} x_{t}^{2}\right)\right. \\
&+ \frac{M^{2}}{256}\left(\frac{31}{8} \frac{x_{H}}{c_{\theta}^{4}}-\frac{15}{2} \frac{x_{H} x_{t}}{c_{\theta}^{2}}+\frac{13}{2} \frac{x_{H}}{c_{\theta}^{2}}-\frac{9}{2} \frac{x_{H}^{2}}{c_{\theta}^{2}}-15 x_{H} x_{t}\right. \\
&+\left.\left.17 x_{H}+9 x_{H}^{2} x_{t}-9 x_{H}^{2}+\frac{27}{8} x_{H}^{3}\right)-\frac{3}{32} \frac{g_{S}^{2}}{g^{2}} s x_{t}\right] \\
& G_{U V ; \text { irr } ; 2}^{H H ; 2}(s)=\frac{g^{4}}{\pi^{4}}\left[\frac{s}{512}\left(-\frac{7}{3}+\frac{431}{48} \frac{1}{c_{\theta}^{4}}+\frac{85}{24} \frac{x_{t}}{c_{\theta}^{2}}-\frac{101}{6} \frac{1}{c_{\theta}^{2}}+\frac{3}{16} x_{H}^{2}+\frac{25}{12} x_{t}-\frac{27}{8} x_{t}^{2}\right)\right. \\
&\left.+\frac{M^{2}}{256}\left(\frac{21}{16} \frac{x_{H}}{c_{\theta}^{4}}-\frac{9}{4} \frac{x_{H}}{c_{\theta}^{2}}+\frac{3}{2} \frac{x_{H}^{2}}{c_{\theta}^{2}}+\frac{3}{2} x_{H}+\frac{9}{4} x_{H}^{2} x_{t}+3 x_{H}^{2}-\frac{9}{16} x_{H}^{3}\right)+\frac{5}{128} \frac{g_{S}^{2}}{g^{2}} s x_{t}\right], \\
& G_{U V ; \text { irr } ; 3}^{H H ; 2}= \frac{g^{4}}{\pi^{4}}\left[\frac{s}{128}\left(1-\frac{43}{32} \frac{1}{c_{\theta}^{4}}-\frac{5}{32} \frac{x_{t}}{c_{\theta}^{2}}+\frac{37}{16} \frac{1}{c_{\theta}^{2}}+\frac{7}{16} x_{t}+\frac{9}{32} x_{t}^{2}\right)\right. \\
&+ \frac{M^{2}}{1024}\left(\frac{31}{8} \frac{x_{H}}{c_{\theta}^{4}}-\frac{15}{2} \frac{x_{H} x_{t}}{c_{\theta}^{2}}+\frac{13}{2} \frac{x_{H}}{c_{\theta}^{2}}-\frac{9}{2} \frac{x_{H}^{2}}{c_{\theta}^{2}}-15 x_{H} x_{t}\right. \\
&+\left.\left.23 x_{H}+9 x_{H}^{2} x_{t}-9 x_{H}^{2}+\frac{27}{8} x_{H}^{3}\right)-\frac{3}{128} \frac{g_{S}^{2}}{g^{2}} s x_{t}\right] .
\end{aligned}
$$

Therefore, we can employ a non-local subtraction term,

$$
G_{U V ; \text { irr } ; a}^{H H ; 2 ; c t}(s)=\frac{g^{4}}{256 \pi^{4}}\left[s \Delta Z_{H ; a}^{(2)}-M_{H}^{2}\left(\Delta Z_{H ; a}^{(2)}+\Delta Z_{M_{H} ; a}^{(2)}\right)\right] .
$$

\section{Two-Loop Counterterms}

In this Section we provide the full list of two-loop counterterms (for reason of space fermion masses other than the top-quark one are not shown, except for the first result).

Let us start with field counterterms, where the sum over the fermion masses was defined in Eq.(84),

$$
\begin{gathered}
\Delta Z_{A A ; 1}^{(2)}=\frac{25}{3} s_{\theta}^{2}, \\
\Delta Z_{A A ; 2}^{(2)}=\frac{923}{108}-\frac{113}{9} \frac{1}{c_{\theta}^{2}}+\frac{13}{6} s_{\theta}^{2} X_{u}+\frac{7}{6} s_{\theta}^{2} X_{d}+\frac{3}{2} s_{\theta}^{2} X_{l}-\frac{127}{108} c_{\theta}^{2}+\frac{140}{27} c_{\theta}^{4}-\frac{64}{9} s_{\theta}^{2} \frac{g_{S}^{2}}{g^{2}} \\
\Delta Z_{A A ; 3}^{(2)}=\frac{15}{4} s_{\theta}^{2} . \\
\Delta Z_{A Z ; 1}^{(2)}=s_{\theta}\left(\frac{943}{9} \frac{1}{c_{\theta}}-214 c_{\theta}+\frac{1058}{9} c_{\theta}^{3}\right), \\
\Delta Z_{A Z ; 2}^{(2)}=s_{\theta}\left(\frac{199}{18} \frac{1}{c_{\theta}^{3}}-\frac{17}{12} \frac{x_{t}}{c_{\theta}}-\frac{172}{9} \frac{1}{c_{\theta}}+\frac{13}{6} c_{\theta} x_{t}-\frac{331}{36} c_{\theta}+\frac{44}{3} \frac{1}{c_{\theta}} \frac{g_{S}^{2}}{g^{2}}-\frac{80}{3} c_{\theta} \frac{g_{S}^{2}}{g^{2}}\right), \\
\Delta Z_{A Z ; 3}^{(2)}=s_{\theta}\left(\frac{943}{9} \frac{1}{c_{\theta}}-\frac{311}{6} c_{\theta}+\frac{529}{18} c_{\theta}^{3}\right) . \\
\Delta Z_{Z ; 1}^{(2)}=\frac{1}{9}\left(5453-1681 \frac{1}{c_{\theta}^{2}}-5813 c_{\theta}^{2}+2116 c_{\theta}^{4}\right),
\end{gathered}
$$




$$
\begin{gathered}
\Delta Z_{Z ; 2}^{(2)}=-\frac{77}{3}-\frac{199}{18} \frac{1}{c_{\theta}^{4}}+\frac{17}{12} \frac{x_{t}}{c_{\theta}^{2}}+\frac{86}{3} \frac{1}{c_{\theta}^{2}}+\frac{13}{6} c_{\theta}^{2} x_{t}-\frac{331}{36} c_{\theta}^{2}-\frac{17}{6} x_{t} \\
-\frac{44}{3} \frac{1}{c_{\theta}^{2}} \frac{g_{S}^{2}}{g^{2}}-\frac{80}{3} c_{\theta}^{2} \frac{g_{S}^{2}}{g^{2}}+\frac{88}{3} \frac{g_{S}^{2}}{g^{2}}, \\
\Delta Z_{Z ; 3}^{(2)}=\frac{5453}{36}-\frac{1681}{36} \frac{1}{c_{\theta}^{2}}-\frac{5753}{36} c_{\theta}^{2}+\frac{529}{9} c_{\theta}^{4} . \\
\Delta Z_{W ; 1}^{(2)}=\frac{25}{3}, \quad \Delta Z_{W}^{(2)}=-\frac{63}{4}-\frac{3}{2} \frac{1}{c_{\theta}^{2}}+\frac{3}{4} x_{t}-12 \frac{g_{S}^{2}}{g^{2}}, \quad \Delta Z_{W ; 3}^{(2)}=\frac{15}{4} . \\
\Delta Z_{H ; 1}^{(2)}=4+\frac{43}{4} \frac{1}{c_{\theta}^{4}}+\frac{5}{4} \frac{x_{t}}{c_{\theta}^{2}}-\frac{37}{2} \frac{1}{c_{\theta}^{2}}-\frac{7}{2} x_{t}-\frac{9}{4} x_{t}^{2}+24 x_{t} \frac{g_{S}^{2}}{g^{2}}, \\
\Delta Z_{H ; 3}^{(2)}=-2+\frac{43}{16} \frac{1}{c_{\theta}^{4}}+\frac{5}{16} \frac{x_{t}}{c_{\theta}^{2}}-\frac{37}{8} \frac{1}{c_{\theta}^{2}}-\frac{7}{8} x_{t}-\frac{9}{16} x_{t}^{2}+6 x_{t} \frac{g_{S}^{2}}{g^{2}} . \\
\Delta Z_{H ; 2}^{(2)}=\frac{7}{6}-\frac{431}{96} \frac{1}{c_{\theta}^{4}}-\frac{85}{48} \frac{x_{t}}{c_{\theta}^{2}}+\frac{101}{12} \frac{1}{c_{\theta}^{2}}-\frac{25}{24} x_{t}+\frac{27}{16} x_{t}^{2}-\frac{3}{32} x_{H}^{2}-10 x_{t} \frac{g_{S}^{2}}{g^{2}},
\end{gathered}
$$

For the $W$-mass counterterm we get

$$
\begin{aligned}
& \Delta Z_{M ; 1}^{(2)}=\frac{121}{9}+9 \frac{1}{c_{\theta}^{8} x_{H}^{2}}-50 \frac{1}{c_{\theta}^{6} x_{H}}+18 \frac{x_{t}}{c_{\theta}^{4} x_{H}}-72 \frac{x_{t}^{2}}{c_{\theta}^{4} x_{H}^{2}}+36 \frac{1}{c_{\theta}^{4} x_{H}^{2}}+59 \frac{1}{c_{\theta}^{4} x_{H}}+\frac{145}{8} \frac{1}{c_{\theta}^{4}} \\
& -\frac{1}{4} \frac{x_{t}}{c_{\theta}^{2}}+2 \frac{x_{t}^{2}}{c_{\theta}^{2} x_{H}}-40 \frac{1}{c_{\theta}^{2} x_{H}}-\frac{27}{2} \frac{1}{c_{\theta}^{2}}+36 \frac{x_{t}}{x_{H}}-\frac{23}{2} x_{t}-144 \frac{x_{t}^{2}}{x_{H}^{2}}+72 \frac{x_{t}^{2}}{x_{H}} \\
& -\frac{117}{4} x_{t}^{2}-18 \frac{x_{t}^{3}}{x_{H}}+144 \frac{x_{t}^{4}}{x_{H}^{2}}+36 \frac{1}{x_{H}^{2}}-8 \frac{1}{x_{H}}-\frac{5}{2} x_{H}-\frac{9}{8} x_{H}^{2}+24 x_{t} \frac{g_{S}^{2}}{g^{2}}-192 \frac{x_{t}^{2}}{x_{H}} \frac{g_{S}^{2}}{g^{2}}, \\
& \Delta Z_{M ; 2}^{(2)}=\frac{149}{12}+\frac{379}{12} \frac{1}{c_{\theta}^{6} x_{H}}+\frac{19}{2} \frac{x_{t}}{c_{\theta}^{4} x_{H}}-\frac{289}{6} \frac{1}{c_{\theta}^{4} x_{H}}-\frac{701}{96} \frac{1}{c_{\theta}^{4}} \\
& -40 \frac{x_{t}}{c_{\theta}^{2} x_{H}}-\frac{85}{48} \frac{x_{t}}{c_{\theta}^{2}}+\frac{8}{3} \frac{x_{t}^{2}}{c_{\theta}^{2} x_{H}}+\frac{77}{3} \frac{1}{c_{\theta}^{2} x_{H}}-\frac{3}{4} \frac{x_{H}}{c_{\theta}^{2}}+\frac{73}{6} \frac{1}{c_{\theta}^{2}}+35 \frac{x_{t}}{x_{H}}+\frac{9}{4} x_{t} x_{H}-\frac{43}{24} x_{t}-\frac{8}{3} \frac{x_{t}^{2}}{x_{H}} \\
& -\frac{21}{16} x_{t}^{2}-15 \frac{x_{t}^{3}}{x_{H}}-\frac{256}{3} \frac{1}{x_{H}}-\frac{3}{2} x_{H}+\frac{63}{32} x_{H}^{2}-10 x_{t} \frac{g_{S}^{2}}{g^{2}}+32 \frac{x_{t}^{2}}{x_{H}} \frac{g_{S}^{2}}{g^{2}}+12 \frac{g_{S}^{2}}{g^{2}}, \\
& \Delta Z_{M ; 3}^{(2)}=-\frac{101}{36}+\frac{9}{4} \frac{1}{c_{\theta}^{8} x_{H}^{2}}-\frac{25}{2} \frac{1}{c_{\theta}^{6} x_{H}}+\frac{9}{2} \frac{x_{t}}{c_{\theta}^{4} x_{H}}-18 \frac{x_{t}^{2}}{c_{\theta}^{4} x_{H}^{2}}+9 \frac{1}{c_{\theta}^{4} x_{H}^{2}}+\frac{59}{4} \frac{1}{c_{\theta}^{4} x_{H}}+\frac{145}{32} \frac{1}{c_{\theta}^{4}} \\
& -\frac{1}{16} \frac{x_{t}}{c_{\theta}^{2}}+\frac{1}{2} \frac{x_{t}^{2}}{c_{\theta}^{2} x_{H}}-4 \frac{1}{c_{\theta}^{2} x_{H}}-\frac{27}{8} \frac{1}{c_{\theta}^{2}}+9 \frac{x_{t}}{x_{H}}-\frac{23}{8} x_{t}-36 \frac{x_{t}^{2}}{x_{H}^{2}}+18 \frac{x_{t}^{2}}{x_{H}}-\frac{117}{16} x_{t}^{2} \\
& -\frac{9}{2} \frac{x_{t}^{3}}{x_{H}}+36 \frac{x_{t}^{4}}{x_{H}^{2}}+9 \frac{1}{x_{H}^{2}}+10 \frac{1}{x_{H}}-\frac{5}{8} x_{H}-\frac{9}{32} x_{H}^{2}+6 x_{t} \frac{g_{S}^{2}}{g^{2}}-48 \frac{x_{t}^{2}}{x_{H}} \frac{g_{S}^{2}}{g^{2}} \text {. }
\end{aligned}
$$


For the cosine of the weak-mixing angle we obtain

$$
\begin{gathered}
\Delta Z_{c_{\theta} ; 1}^{(2)}=\frac{11911}{72}-\frac{1681}{72} \frac{1}{c_{\theta}^{4}}-\frac{205}{36} \frac{1}{c_{\theta}^{2}}-\frac{2021}{9} c_{\theta}^{2}+\frac{529}{6} c_{\theta}^{4}, \\
\Delta Z_{c_{\theta} ; 2}^{(2)}=-\frac{119}{24}-\frac{199}{36} \frac{1}{c_{\theta}^{4}}+\frac{17}{24} \frac{x_{t}}{c_{\theta}^{2}}+\frac{181}{12} \frac{1}{c_{\theta}^{2}} \\
+\frac{13}{12} c_{\theta}^{2} x_{t}-\frac{331}{72} c_{\theta}^{2}-\frac{43}{24} x_{t}-\frac{22}{3} \frac{1}{c_{\theta}^{2}} \frac{g_{S}^{2}}{g^{2}}-\frac{40}{3} c_{\theta}^{2} \frac{g_{S}^{2}}{g^{2}}+\frac{62}{3} \frac{g_{S}^{2}}{g^{2}}, \\
\Delta Z_{c_{\theta} ; 3}^{(2)}=\frac{11671}{288}-\frac{1681}{288} \frac{1}{c_{\theta}^{4}}-\frac{205}{144} \frac{1}{c_{\theta}^{2}}-\frac{1991}{36} c_{\theta}^{2}+\frac{529}{24} c_{\theta}^{4} .
\end{gathered}
$$

Counterterms for the Higgs-boson mass read as

$$
\begin{aligned}
\Delta Z_{M_{H} ; 1}^{(2)} & =13-\frac{55}{8} \frac{1}{c_{\theta}^{4}}-\frac{35}{4} \frac{x_{t}}{c_{\theta}^{2}}-\frac{9}{2} \frac{x_{H}}{c_{\theta}^{2}}+25 \frac{1}{c_{\theta}^{2}}+9 x_{t} x_{H} \\
& -\frac{23}{2} x_{t}+\frac{9}{4} x_{t}^{2}-9 x_{H}+\frac{27}{8} x_{H}^{2}-24 x_{t} \frac{g_{S}^{2}}{g^{2}}, \\
\Delta Z_{M_{H} ; 2}^{(2)}= & \frac{1}{3}+\frac{557}{96} \frac{1}{c_{\theta}^{4}}+\frac{85}{48} \frac{x_{t}}{c_{\theta}^{2}}+\frac{3}{2} \frac{x_{H}}{c_{\theta}^{2}}-\frac{32}{3} \frac{1}{c_{\theta}^{2}}+\frac{9}{4} x_{t} x_{H} \\
& +\frac{25}{24} x_{t}-\frac{27}{16} x_{t}^{2}+3 x_{H}-\frac{15}{32} x_{H}^{2}+10 x_{t} \frac{g_{S}^{2}}{g^{2}}, \\
\Delta Z_{M_{H} ; 3}^{(2)} & =\frac{31}{4}-\frac{55}{32} \frac{1}{c_{\theta}^{4}}-\frac{35}{16} \frac{x_{t}}{c_{\theta}^{2}}-\frac{9}{8} \frac{x_{H}}{c_{\theta}^{2}}+\frac{25}{4} \frac{1}{c_{\theta}^{2}}+\frac{9}{4} x_{t} x_{H} \\
& -\frac{23}{8} x_{t}+\frac{9}{16} x_{t}^{2}-\frac{9}{4} x_{H}+\frac{27}{32} x_{H}^{2}-6 x_{t} \frac{g_{S}^{2}}{g^{2}} .
\end{aligned}
$$

Here we present also the two-loop counterterms for the Higgs-Kibble $\varphi^{0}$ and $\varphi$ scalar bosons,

$$
\begin{gathered}
\Delta Z_{\varphi^{0} ; 1}^{(2)}=\Delta Z_{\varphi ; 1}^{(2)}=4+\frac{43}{4} \frac{1}{c_{\theta}^{4}}+\frac{5}{4} \frac{x_{t}}{c_{\theta}^{2}}-\frac{37}{2} \frac{1}{c_{\theta}^{2}}-\frac{7}{2} x_{t}-\frac{9}{4} x_{t}^{2}+24 x_{t} \frac{g_{S}^{2}}{g^{2}}, \\
\Delta Z_{\varphi^{0} ; 2}^{(2)}=\Delta Z_{\varphi ; 2}^{(2)}=\frac{7}{6}-\frac{431}{96} \frac{1}{c_{\theta}^{4}}-\frac{85}{48} \frac{x_{t}}{c_{\theta}^{2}}+\frac{101}{12} \frac{1}{c_{\theta}^{2}}-\frac{25}{24} x_{t}+\frac{27}{16} x_{t}^{2}-\frac{3}{32} x_{H}^{2}-10 x_{t} \frac{g_{S}^{2}}{g^{2}}, \\
\Delta Z_{\varphi^{0} ; 3}^{(2)}=\Delta Z_{\varphi ; 3}^{(2)}=-2+\frac{43}{16} \frac{1}{c_{\theta}^{4}}+\frac{5}{16} \frac{x_{t}}{c_{\theta}^{2}}-\frac{37}{8} \frac{1}{c_{\theta}^{2}}-\frac{7}{8} x_{t}-\frac{9}{16} x_{t}^{2}+6 x_{t} \frac{g_{S}^{2}}{g^{2}} .
\end{gathered}
$$

The equalities in Eq.(86) and in Eqs.(214) -(216) are related to the custodial symmetry $S U(2)_{L} \otimes S U(2)_{R} \rightarrow$ $S U(2)_{V}$. Finally, gauge-parameter counterterms can be expressed by means of Eqs.(192)-(216), similarly to the one-loop case,

$$
\begin{aligned}
\delta Z_{\xi_{A}}^{(2)}=\frac{1}{2}\left\{\delta Z_{A A}^{(2)}-\frac{1}{4}\left[\delta Z_{A A}^{(1)}\right]^{2}\right\}, & \delta Z_{\xi_{Z}}^{(2)}=\frac{1}{2}\left\{\delta Z_{Z}^{(2)}-\frac{1}{4}\left[\delta Z_{Z}^{(1)}\right]^{2}\right\} \\
\delta Z_{\xi_{W}}^{(2)}=\frac{1}{2}\left\{\delta Z_{W}^{(2)}-\frac{1}{4}\left[\delta Z_{W}^{(1)}\right]^{2}\right\}, & \delta Z_{\xi_{A Z}}^{(2)}=-\delta Z_{A Z}^{(2)}+\frac{1}{2} \delta Z_{A Z}^{(1)}\left[\delta Z_{A A}^{(1)}+\delta Z_{Z}^{(1)}\right],
\end{aligned}
$$




$$
\begin{aligned}
\delta Z_{\xi_{\varphi}}^{(2)} & =-\frac{1}{2}\left[\delta Z_{\varphi}^{(2)}+\delta Z_{M}^{(2)}\right]+\frac{3}{8}\left\{\left[\delta Z_{\varphi}^{(1)}\right]^{2}+\left[\delta Z_{M}^{(1)}\right]^{2}\right\}+\frac{1}{4} \delta Z_{\varphi}^{(1)} \delta Z_{M}^{(1)}, \\
\delta Z_{\xi_{\varphi^{0}}}^{(2)} & =-\frac{1}{2}\left[\delta Z_{\varphi^{0}}^{(2)}+\delta Z_{M}^{(2)}-2 \delta Z_{c_{\theta}}^{(2)}\right]+\frac{3}{8}\left\{\left[\delta Z_{\varphi^{0}}^{(1)}\right]^{2}+\left[\delta Z_{M}^{(1)}\right]^{2}\right\} \\
& +\frac{1}{4} \delta Z_{\varphi^{0}}^{(1)} \delta Z_{M}^{(1)}-\frac{1}{2} \delta Z_{c_{\theta}}^{(1)}\left[\delta Z_{\varphi^{0}}^{(1)}+\delta Z_{M}^{(1)}\right] .
\end{aligned}
$$

After deriving the relations for the two-loop gauge-parameter counterterms, we can verify immediately an important result: the doubly-contracted WST identities with two external gauge-boson fields have the same form derived in 't Hooft-Feynman gauge. The gauge-fixing functions given in Eq.(58) are expressed by means of bare quantities. After introducing renormalized quantities, the gauge-fixing functions change; their expression, order-by-order in perturbation theory, can be read in Eq.(108). By expanding Eq.(108) up to two loops, and using the expression of Eqs.(217)-(218) for the gauge-parameter counterterms, we derive

$$
\mathcal{C}^{A}=-\partial_{\mu} A_{\mu}+\mathcal{O}\left(g^{6}\right), \quad \mathcal{C}^{Z}=-\partial_{\mu} Z_{\mu}+M_{0} \Phi^{0}+\mathcal{O}\left(g^{6}\right), \quad \mathcal{C}^{ \pm}=-\partial_{\mu} W_{\mu}+M \Phi+\mathcal{O}\left(g^{6}\right)
$$

and the doubly-contracted WST identities are formally equivalent to those of Fig. 5 at $\mathcal{O}\left(g^{4}\right)$.

\section{Conclusions}

In this paper, we have collected all the ingredients that are needed to construct a two-loop renormalization procedure for the minimal Standard Model and to work out the details for constructing theoretical predictions for physical (pseudo-)observables.

The procedure covers broadly (and not relying on other sources) all aspects of the problem, from diagram generation, to definition of the renormalization scheme, to evaluation (mostly numerically) of Green functions, including all customary approximations that are usually made as the small fermion-mass approximation (with particular attention to the analytical extraction of collinear logarithms). Our scheme can deal with arbitrary scales and goes beyond the introduction of $\overline{M S}$ couplings in the electroweak sector.

Among the technical aspects which we consider to be innovative and that we have introduced in this paper we point out a universal ultraviolet decomposition of one- and two-loop diagrams which is closed with respect to multiplication and a non-negligible simplification in the structure of renormalized Green functions due to the (possible) introduction of a non-minimal $\overline{M S}$ prescription. We also present residues of ultraviolet poles, up to two-loop vertices, in terms of familiar one-loop functions.

Techniques for expanding one- and two-loop diagrams in different regions, small fermion masses or small momentum transfer or both, have been introduced and discussed as well as their relevance to renormalization.

Applications, as well as the introduction of unstable particles in the renormalization procedure, will be presented in a third, forthcoming, paper.

In several appendices we have presented examples of our computational techniques. Although there is no substitute for the original FORM codes in modern technology, we are confident enough that the examples shown here suffice in illustrating our methods. The parallel development of $\mathcal{G}$ raphShot and of $\mathcal{L}$ oop $\mathcal{B} a c k$ is aimed to give to most complete documentation for obtaining all results which are too long to be presented here; if we ask the question 'might the theory in general be a lot simpler than we thought?' we may conclude that we have a 'nearly nifty' formulation 2 showing that the Standard Model is canonically as simple as QED, even at the two-loop level.

\section{Acknowledgments}

The contribution of Andrea Ferroglia and Massimo Passera to an early stage of this paper is gratefully acknowledged. We recognize the role played by Sandro Uccirati in several steps of our global project. Finally, we gratefully acknowledge several discussions with Ansgar Denner, Stefan Dittmaier and Mikhail Kalmykov. S.A. is indebted to N. Glover for hospitality at the Institute for Particle Physics Phenomenology of the University of Durham where part of the manuscript was written.

\footnotetext{
${ }^{2}$ see Z. Bern talk, High precision for hard processes at the LHC, Zurich 2006
} 

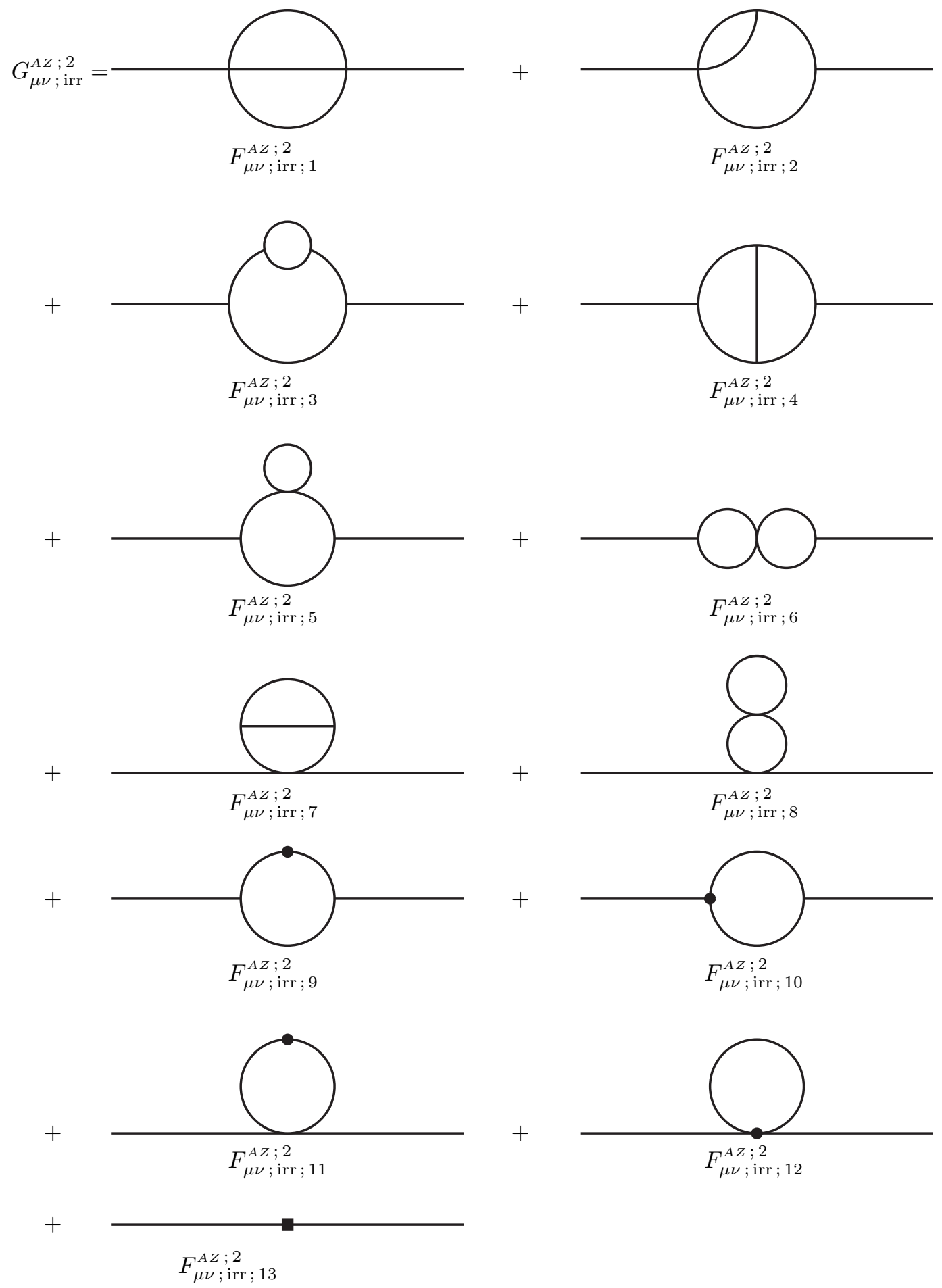

Figure 14: The irreducible contributions to the Green function with one external photon and one external $Z$ boson at $\mathcal{O}\left(g^{4}\right)$ (denoted by the superscript 2). Thirteen families of Feynman diagrams give a contribution (each gets a subscript-label). Dotted vertices depend on $\beta_{1}, \Gamma_{1}$ or the counterterms at $\mathcal{O}\left(g^{2}\right)$. The black-square vertex represents a vertex which depends on $\Gamma_{2}$ or the counterterms at $\mathcal{O}\left(g^{4}\right)$. 

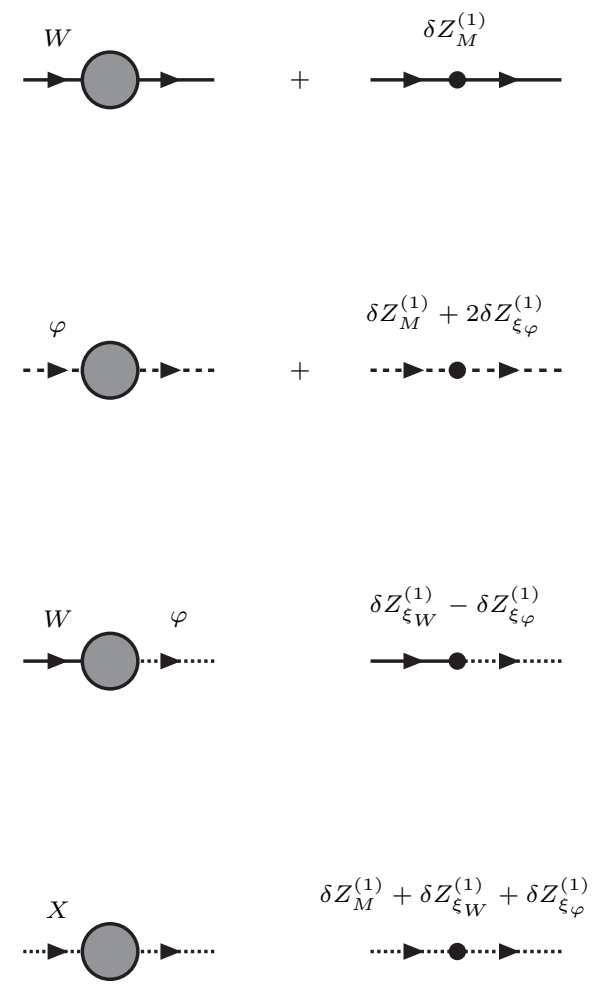

Figure 15: The correct recipe for renormalization of mass-dependent UV poles in the charged sector. 


\section{A Reduction of vacuum bubbles}

Following the definitions of Section 3.3 we give few examples of relations between two-loop vacuum bubbles and the master bubble, which we select to be $T_{121}$. For instance, we have

$$
\begin{aligned}
T_{211}\left(m_{3}, m_{1}, m_{2}\right) & =-\frac{1}{2 m_{3}^{2}}\left[A_{0}\left(1, m_{1}\right) A_{0}\left(2, m_{2}\right)+A_{0}\left(1, m_{3}\right) A_{0}\left(2, m_{2}\right)\right. \\
& \left.+(n-3) T_{111}\left(m_{3}, m_{1}, m_{2}\right)+\left(m_{3}^{2}+m_{2}^{2}-m_{1}^{2}\right) T_{112}\left(m_{3}, m_{1}, m_{2}\right)\right] \\
T_{112}\left(m_{3}, m_{1}, m_{2}\right) & =-\frac{1}{m_{1}^{2}-m_{2}^{2}-m_{3}^{2}}\left[A_{0}\left(1, m_{2}\right) A_{0}\left(2, m_{1}\right)\right. \\
& -A_{0}\left(1, m_{1}\right) A_{0}\left(2, m_{2}\right)-A_{0}\left(1, m_{3}\right) A_{0}\left(2, m_{1}\right)+A_{0}\left(1, m_{3}\right) A_{0}\left(2, m_{2}\right) \\
& \left.+\left(m_{3}^{2}-m_{2}^{2}+m_{1}^{2}\right) T_{121}\left(m_{3}, m_{1}, m_{2}\right)\right], \\
(2-n) T_{111}\left(m_{3}, m_{1}, m_{2}\right) & =\frac{1}{m_{1}^{2}-m_{2}^{2}-m_{3}^{2}} \\
& \times\left\{\left(m_{1}^{2}+m_{2}^{2}-m_{3}^{2}\right)\left[A_{0}\left(1, m_{1}\right) A_{0}\left(2, m_{2}\right)+A_{0}\left(2, m_{1}\right) A_{0}\left(1, m_{3}\right)\right]\right. \\
& -\left(m_{1}^{2}+m_{2}^{2}-m_{3}^{2}\right)\left[A_{0}\left(2, m_{2}\right) A_{0}\left(1, m_{3}\right)+A_{0}\left(2, m_{1}\right) A_{0}\left(1, m_{2}\right)\right] \\
& \left.+\left[\left(m_{2}^{2}-m_{3}^{2}\right)^{2}-m_{1}^{4}\right] T_{121}\left(m_{3}, m_{1}, m_{2}\right)\right\}+A_{0}\left(1, m_{3}\right) A_{0}\left(2, m_{2}\right) \\
& -A_{0}\left(1, m_{1}\right) A_{0}\left(2, m_{2}\right)+2 m_{1}^{2} T_{121}\left(m_{3}, m_{1}, m_{2}\right) .
\end{aligned}
$$

Vacuum bubbles with arbitray powers can always be reduced to a combination of products of $A_{0}$ functions and of $T_{121}$. This results are relevant for processes where all external scales can be put to zero.

An efficient algorithmic solution of the IBP equations for vacuum bubbles must be able to isolate special cases; an example is given by the following two cases:

$$
\begin{aligned}
T_{112}(0, m, m) & =\frac{1}{2 m^{2}} A_{0}(1, m) A_{0}(2, m), \\
T_{112}\left(0, m_{1}, m_{2}\right) & =2 \frac{m_{2}^{2}}{m_{1}^{2}} A_{0}\left(1, m_{1}\right) A_{0}\left(3, m_{2}\right)-A_{0}\left(2, m_{1}\right) A_{0}\left(2, m_{2}\right)-T_{121}\left(0, m_{1}, m_{2}\right) .
\end{aligned}
$$

We can now compute the master vacuum bubble of Eq.(30), which is representable as

$$
\begin{gathered}
T_{121}^{\mathrm{fin}}\left(m_{1}, m_{2}, m_{3}\right) \equiv T_{121}\left(m_{1}, m_{2}, m_{3} ; 0\right)=\frac{7}{2}-\frac{1}{2} \zeta(2)-\ln \frac{m_{2}^{2}}{M^{2}}\left(1+\frac{1}{2} \ln \frac{m_{2}^{2}}{M^{2}}\right)+\int_{0}^{1} d x T(x), \\
T(x)=-\frac{Q(x)}{P(x)}\left[\ln m_{2}^{2}-\ln \frac{Q(x)}{X}\right]-\ln |P(x)|\left[\ln m_{2}^{2}+\ln X\right]+\frac{1}{2} \ln ^{2}|P(x)|+\operatorname{Li}_{2}\left(\frac{m_{2}^{2}}{M^{2}} \frac{X}{P(x)}\right), \\
Q(x)=\frac{m_{1}^{2}-m_{3}^{2}}{M^{2}} x-\frac{m_{1}^{2}}{M^{2}}, \quad P(x)=Q(x)+\frac{m_{2}^{2}}{M^{2}} X,
\end{gathered}
$$

with $X=x(1-x)$; special cases are given in Appendix $\mathbf{C}$

\section{B Singularities whose origin is infrared}

As mentioned in Section 2 after reduction we may have a migration of poles, from the IR to the UV; in this appendix we collect two examples. 
B.1 Evaluation of $s^{C}\left(s=0 ; m_{1}, m_{2}, 0,0\right)$

In this appendix we consider the following integral, belonging to the $C$-family (Sect. 5 of Ref. [12]),

$$
\begin{aligned}
s^{C}\left(s=0 ; m_{1}, m_{2}, 0,0\right) & =-2\left(\pi M^{2}\right)^{-\epsilon} \Gamma(\epsilon) \int_{0}^{1} d x \int_{0}^{1} d y \int_{0}^{y} d z[x(1-x)]^{-\epsilon / 2}(1-y)^{\epsilon / 2} \\
& \times\left[m_{x}^{2} V_{C}^{-1-\epsilon}+\left(\frac{2}{\epsilon}-\frac{1}{2}\right) V_{C}^{-\epsilon}\right] .
\end{aligned}
$$

When $s=0$ and $m_{3}=m_{4}=0$ we have $V_{C}=(1-y) m_{x}^{2}$ and the first term in square brackets in Eq.(225) is singular at $y=1$. Furthermore,

$$
x(1-x) m_{x}^{2}=\frac{m_{1}^{2}}{M^{2}}+\frac{m_{2}^{2}-m_{1}^{2}}{M^{2}} x .
$$

We now perform the (trivial) $z$ integration and the $y$ integration using, e.g.,

$$
\int_{0}^{1}(1-y)^{-1-\epsilon / 2}=-\frac{2}{\epsilon}
$$

where we have naively assumed the relation $\epsilon_{U V}=-\epsilon_{I R}$. The last $x$ integration is finally performed by using the following relation, valid for any monomial $V(x)$,

$$
V^{-1-\epsilon / 2}(x)=\frac{M^{2}}{m_{1}^{2}}\left[1+\frac{x}{\epsilon} \frac{d}{d x}\right] V^{-\epsilon}(x) .
$$

Now, after integration by parts we obtain

$$
\frac{\left(\pi M^{2}\right)^{\epsilon}}{\Gamma(1+\epsilon)} s^{C}\left(0, m_{1}, m_{2}, 0,0\right)=\frac{2}{\epsilon^{2}}+\left[1+2 \frac{m_{1}^{2}}{m_{2}^{2}-m_{1}^{2}} \ln \frac{m_{1}^{2}}{M^{2}}-2 \frac{m_{2}^{2}}{m_{2}^{2}-m_{1}^{2}} \ln \frac{m_{2}^{2}}{M^{2}}\right] \frac{1}{\epsilon}+\mathcal{O}(1) .
$$

Note that the residue of the double pole has changed sign with respect to the non-IR configuration.

\section{B.2 Evaluation of $s^{E}\left(s, m_{1}, m_{2}, 0, m_{4}, 0\right)$}

Another example is represented by $s^{E}\left(s, m_{1}, m_{2}, 0, m_{4}, 0\right)$ where we have to compute the following threefold integral,

$$
\int_{0}^{1} d x \int_{0}^{1} d y \int_{0}^{y} d z[x(1-x)]^{-\epsilon / 2}(1-y)^{\epsilon / 2}(y-z) V_{E}^{-1-\epsilon}
$$

where the quadratic form $V_{E}$ (see Sect. 6 of Ref. [12]) is defined as,

$$
V_{E}=z^{2}-a z+b(1-y), \quad a=1-\frac{m_{4}^{2}}{s}, \quad b=m_{x}^{2} \frac{M^{2}}{s},
$$

with $m_{x}^{2}$ given in Eq.(226). After changing variable, $y^{\prime}=1-y$, in Eq.(230) we use a functional relation valid for quadratic forms,

$$
V^{-1-\epsilon}=-\frac{4}{a^{2}}\left[1+\frac{y}{\epsilon} \partial_{y}+\frac{1}{2}\left(z-\frac{a}{2}\right) \partial_{z}\right] V^{-\epsilon} .
$$

After inserting this result in Eq.(230) and after integration by parts we obtain the result,

$$
\begin{aligned}
\frac{(\pi s)^{\epsilon}}{\Gamma(1+\epsilon)} s^{E}\left(s, m_{1}, m_{2}, 0, m_{4}, 0\right) & =-\frac{2}{a} \frac{1}{\epsilon^{2}}+\left[-\frac{1}{a}+\left(1-\frac{1}{a}\right) \ln (1-a)-\left(1-\frac{2}{a}\right) \ln (-a)\right. \\
& \left.-\frac{m_{1}^{2}}{m_{2}^{2}-m_{1}^{2}} \ln \frac{m_{1}^{2}}{s}+\frac{m_{2}^{2}}{m_{2}^{2}-m_{1}^{2}} \ln \frac{m_{2}^{2}}{s}\right] \frac{1}{\epsilon}+\mathcal{O}(1),
\end{aligned}
$$

where, once more, $a=1-m_{4}^{2} / s$; this function, for a non-IR configuration $\left(m_{3} \neq 0\right)$, shows only a single pole at $n=4$; in the case $m_{3}=0$ (coming from reduction of integrals with irreducible scalar products in the numerator) there is, instead, a double pole. 
Note that all these features arise from the reduction of $S_{p p}^{E}(1,1, \cdots)$ and of $S_{d}^{E}(1,1, \cdots)$; in the case of pure QED all these terms are absent and, as a matter of fact, they are also absent in the doubly contracted Ward identity for the $A A$ transition, even in the full standard model. It is only in the evaluation of $\Pi(s)-$ vacuum polarization in the full standard model - that they are needed; here, for the first time, the explicit form of the two-loop, two-point, form factors is actually needed and represents a severe test for the final result.

There are virtues and vices in any reduction algorithm; note, however, that a check on any calculation cannot do without reduction, i.e. without the possibility of expressing the result in terms of a basis.

\section{The limit $m_{f} \rightarrow 0$}

All diagrams contributing to a certain Green function are generated by our code $\mathcal{G}$ raphShot; the output of $\mathcal{G}$ raphShot is fully general and fermion masses are kept in the final answer. However, in most cases, we are interested in taking the limit $m_{f} \rightarrow 0, f \neq t$, extracting at the same time all the collinear logarithms.

After renormalization, we aim to compute physical observables with an algebraic-numerical approach; all contributions that are potentially large, e.g. collinear logarithms, are extracted with the use of an analytical calculation and only the collinear-free reminder is subject to a numerical evaluation. In few cases even this last step can be avoided given that also the finite part (in the collinear limit) is expressed in terms of polylogarithms. In this way we are always able to produce and explicit proof of the expected cancellations: no power behavior in a gauge theory etc.

Collinear logarithms has two different origins; for instance they are present in the vacuum polarization function due to QED effects; for two loop irreducible contributions, however, they are sub-leading; e.g. the correction factor is proportional to $g^{4} \ln m_{l}^{2}$ and not to $g^{4} \ln ^{2} m_{l}^{2}$. Thus, cancellation of the leading part is an important test of the calculation.

Otherwise, collinear logarithms (sometimes even power behavior) may be induced by the reduction procedure in different pieces of the final answer, although the total is collinear free. In this cases, where there is no enhancement, one could as well set $m_{f}$ to zero from the very beginning and control cancellation of collinear poles in $n-4$. The equivalence of the two formulations has not yet received a satisfactory answer.

To discuss the collinear limit we introduce scaled variables,

$$
z_{i}=\frac{m_{i}^{2}}{s}, \quad L_{i}=\ln z_{i}
$$

and collect our results.

\section{- Miscellanea}

The following expansions (see Eq.(21) for the corresponding definitions), which have been implemented in $\mathcal{G}$ raphShot, are very useful in deriving the limit of Green functions for small values of the light fermion masses:

$$
\begin{gathered}
\beta^{-1}\left(z_{f}\right) \ln \frac{\beta\left(z_{f}\right)+1}{\beta\left(z_{f}\right)-1}=-L_{f}-i \pi-2 z_{f}\left(1+L_{f}+i \pi\right)-z_{f}^{2}\left[7+6\left(L_{f}+i \pi\right)\right]+\mathcal{O}\left(z_{f}^{3}\right), \\
\lambda^{1 / 2}\left(1, z_{t}, z_{b}\right)=1-z_{t}-z_{b}+\mathcal{O}\left(z_{b}^{3}\right), \\
\lambda^{-1 / 2}\left(1, z_{t}, z_{b}\right)=-\frac{1}{z_{t}-1}\left[1-\frac{z_{b}}{z_{t}-1}+\left(\frac{z_{b}}{z_{t}-1}\right)^{2}\right]+\mathcal{O}\left(z_{b}^{3}\right),
\end{gathered}
$$

while, for a massive top quark, we obtain

$$
L\left(1, z_{t}, z_{b}\right)=-\frac{1}{2} L_{t}-\frac{1}{2} L_{b}+\ln \left(z_{t}-1\right)-\frac{z_{b}}{\left(z_{t}-1\right)^{2}}-\frac{z_{b}^{2}}{\left(z_{t}-1\right)^{3}}\left(1+\frac{3}{2} \frac{1}{z_{t}-1}\right)+\mathcal{O}\left(z_{b}^{3}\right) .
$$


- $\underline{\mathcal{O}(\epsilon) \text { one-loop functions }}$

$\mathcal{O}(\epsilon)$ one-loop functions, Eqs.(16)-(17), are expanded as follows:

$$
\begin{aligned}
b_{0}^{\epsilon}\left(2,1, s, m_{f}, m_{f}\right) & =4 \zeta(2)+L_{f}^{2}-2 z_{f}\left[2-4 \zeta(2)+L_{f}-L_{f}^{2}\right], \\
b_{0}^{\epsilon}\left(1,1, s, m_{f}, m_{f}\right) & =-8+8 \zeta(2)-2 z_{f}\left[2+8 \zeta(2)-2 L_{f}+L_{f}^{2}\right]-4 i \pi \\
b_{0}^{\epsilon}\left(1,1, s, 0, m_{f}\right) & =-8+8 \zeta(2)-z_{f}\left[2+8 \zeta(2)-2 L_{f}+L_{f}^{2}\right]-4 i \pi, \\
b_{0}^{\mathrm{fin}}\left(1,1, s, m_{b}, m_{t}\right) & =-\frac{1}{2}\left(L_{b}+L_{t}\right)-\frac{1}{2}\left(z_{b}-z_{t}\right)\left(L_{b}-L_{t}\right)+2-\lambda^{1 / 2}\left(1, z_{b}, z_{t}\right) L\left(1, z_{b}, z_{t}\right) . \\
b_{0}^{\epsilon}\left(2,1, s, m_{b}, m_{t}\right) & =-4\left\{-L_{t}+\ln \left(z_{t}-1\right)+\frac{1}{2} b_{0}^{\epsilon}\left(2,1, s, m_{t}, m_{b}\right)\right. \\
+ & \left.\frac{1}{z_{t}-1}\left[-L_{t}+\frac{1}{4} L_{b}^{2}+2+\frac{1}{4} b_{0}^{\epsilon}\left(1,1, s, m_{t}, m_{b}\right)+\frac{1}{2} b_{0}^{\epsilon}\left(2,1, s, m_{t}, m_{b}\right)\right]\right\}, \\
b_{0}^{\epsilon}\left(2,1, s, m_{f}, 0\right) & =2 \operatorname{Li}_{2}\left(\frac{1}{1-z_{f}}\right)-\ln ^{2}\left(z_{f}-1\right)+L_{f}^{2} .
\end{aligned}
$$

- Vacuum bubbles

For vacuum bubbles, see the definition in Eq. (31), we introduce variables

$$
x_{i}=\frac{m_{i}^{2}}{M_{W}^{2}}, \quad \mathcal{L}_{i}=\ln x_{i},
$$

and derive the following results,

$$
\begin{aligned}
T_{f}\left(0, M_{W}, 0\right) & =-\frac{1}{2}-\frac{3}{2} \zeta(2), \\
T_{f}\left(0, M_{Z}, 0\right) & =-\frac{1}{2}-\frac{3}{2} \zeta(2)+\mathcal{L}_{Z}-\mathcal{L}_{Z}^{2}, \\
T_{f}\left(0, m_{t}, 0\right) & =-\frac{1}{2}-\frac{3}{2} \zeta(2)+\mathcal{L}_{t}-\mathcal{L}_{t}^{2}, \\
T_{f}\left(0, m_{f}, M_{W}\right) & =-\frac{1}{2}+\frac{1}{2} \zeta(2)+\mathcal{L}_{f}-\frac{1}{2} \mathcal{L}_{f}^{2}, \\
T_{f}\left(0, m_{f}, M_{Z}\right) & =-\frac{1}{2}+\frac{1}{2} \zeta(2)+\mathcal{L}_{f}\left(1-\mathcal{L}_{Z}-\frac{1}{2} \mathcal{L}_{f}\right)+\frac{1}{2} \mathcal{L}_{Z}^{2}, \\
T_{f}\left(M_{Z}, m_{f}, m_{f}\right) & =-\frac{1}{2}+\frac{1}{2} \zeta(2)+\mathcal{L}_{f}\left(1-\mathcal{L}_{Z}-\frac{1}{2} \mathcal{L}_{f}\right)+\frac{1}{2} \mathcal{L}_{Z}^{2}, \\
T_{f}\left(M_{H}, m_{f}, m_{f}\right) & =-\frac{1}{2}+\frac{1}{2} \zeta(2)+\mathcal{L}_{f}\left(1-\mathcal{L}_{H}-\frac{1}{2} \mathcal{L}_{f}\right)+\frac{1}{2} \mathcal{L}_{H}^{2}, \\
T_{f}\left(0, m_{f}, M_{H}\right) & =-\frac{1}{2}+\frac{1}{2} \zeta(2)+\mathcal{L}_{f}\left(1-\mathcal{L}_{H}-\frac{1}{2} \mathcal{L}_{f}\right)+\frac{1}{2} \mathcal{L}_{H}^{2} .
\end{aligned}
$$

Here, $f$ is a generic light fermion, $f \neq t$. For the $t-b$ doublet we obtain instead,

$$
\begin{aligned}
T_{f}\left(M_{W}, m_{b}, m_{t}\right) & =-\frac{1}{2}-\frac{1}{2} \zeta(2)+\mathcal{L}_{b}-\frac{1}{2} \mathcal{L}_{b}^{2}-\frac{x_{t}}{x_{t}-1}\left[\mathcal{L}_{t} \mathcal{L}_{b}+\frac{1}{2} \mathcal{L}_{t}^{2}+\mathrm{Li}_{2}\left(1-\frac{1}{x_{t}}\right)\right] \\
& -\frac{1}{x_{t}-1} \operatorname{Li}_{2}\left(1-x_{t}\right), \\
T_{f}\left(0, m_{b}, m_{t}\right) & =-\frac{1}{2}+\frac{1}{2} \zeta(2)+\mathcal{L}_{b}\left(1-\frac{1}{2} \mathcal{L}_{b}-\mathcal{L}_{t}\right)+\frac{1}{2} \mathcal{L}_{t}^{2},
\end{aligned}
$$

and the above list includes all possible cases to be found at two loops. 


\section{- Vertices and self-energies}

For all the diagrams at two loops where we have large values of one of the external $\left|p^{2}\right|$ and small values for some of the internal masses it will be convenient to extract the large logarithms by defining regular functions. Note that individual scalar contributions may even show power behavior: such is the case, for instance, for $V_{0}^{M}$ when $p_{1,2}^{2}=0$ and $m_{2}=\ldots=m_{5}=m$ when $m^{2} \ll\left|P^{2}\right|$. In this case the behavior is $\left|P^{2}\right| / m^{2}$.

In computing the vacuum polarization function we have introduced few objects that are special in the sense that some of the masses that appear in the argument list are zero as a consequence of the algorithm of reduction in sub-loops. This special feature modifies the singular structure of the function and introduces finite reminders that should not be confused with the regular ones for which a regulator exists; something that is usually mispresented is that $m=0$ ( $\epsilon$ regulated) is different from $m \rightarrow 0$. Introducing the collinear factor

$$
C_{F}^{f}=-L_{f}+L_{F} L_{f}+\frac{1}{2} L_{f}^{2},
$$

we derive the following results, where $\{0\}_{n}$ stands for $n$ zero masses:

$$
\begin{aligned}
& s^{E ; \text { fin }}\left(s, M_{Z}, m_{f}, m_{f}, m_{f}, m_{f}\right)=s^{E ; \mathrm{reg}}\left(s, M_{Z},\{0\}_{4}\right)+C_{Z}^{f}, \\
& s^{E ; \text { fin }}\left(s, M_{Z}, m_{f}, m_{f}, 0, m_{f}\right)=s^{E ; \operatorname{reg}}\left(s, M_{Z},\{0\}_{4}\right)+C_{Z}^{f} \text {, } \\
& s^{E ; \text { fin }}\left(s, 0, M_{W}, m_{f}, m_{f}, m_{f}\right)=s^{E ; \operatorname{reg}}\left(s, M_{W},\{0\}_{4}\right)+C_{W}^{f} \text {, } \\
& s^{E ; \text { fin }}\left(s, M_{W}, 0, m_{f}, m_{f}, m_{f}\right)=s^{E ; \operatorname{reg}}\left(s, M_{W},\{0\}_{4}\right)+C_{W}^{f} \text {, } \\
& s^{E ; \text { fin }}\left(s, M_{W}, 0, m_{f}, 0, m_{f}\right)=s^{E ; \operatorname{reg}}\left(s, M_{W},\{0\}_{4}\right)+C_{W}^{f}, \\
& s^{E ; \operatorname{spec}}\left(s, m_{f}, m_{f}, 0, M_{W}, 0\right)=s^{E ; \operatorname{spec} R}\left(s,\{0\}_{3}, M_{W}, 0\right)+\frac{L_{f}+L_{f}^{2}}{x_{W}-1} . \\
& s^{E ; \text { fin }}\left(s, M_{W}, m_{u}, m_{d}, m_{u}, m_{d}\right)=s^{E ; \operatorname{reg}}\left(s, M_{W},\{0\}_{4}\right)+C_{W}^{d}, \\
& s^{E ; \text { fin }}\left(s, M_{W}, m_{d}, m_{u}, m_{d}, m_{u}\right)=s^{E ; \operatorname{reg}}\left(s, M_{W},\{0\}_{4}\right)+C_{W}^{u}, \\
& s^{E ; \text { fin }}\left(s, M_{W}, m_{u}, m_{d}, m_{d}, m_{d}\right)=s^{E ; \operatorname{reg}}\left(s, M_{W},\{0\}_{4}\right)+C_{W}^{d}, \\
& s^{E ; \operatorname{fin}}\left(s, M_{W}, m_{d}, m_{u}, m_{u}, m_{u}\right)=s^{E ; \operatorname{reg}}\left(s, M_{W},\{0\}_{4}\right)+C_{W}^{u} \text {, } \\
& s^{E ; \operatorname{fin}}\left(s, M_{Z}, m_{u}, m_{u}, m_{d}, m_{u}\right)=s^{E ; \operatorname{reg}}\left(s, M_{Z},\{0\}_{4}\right)+C_{Z}^{u} \text {, } \\
& s^{E ; \operatorname{fin}}\left(s, M_{z}, m_{u}, m_{u}, m_{u}, m_{u}\right)=s^{E ; \operatorname{reg}}\left(s, M_{Z},\{0\}_{4}\right)+C_{z}^{u} \text {, } \\
& s^{E ; \operatorname{fin}}\left(s, M_{Z}, m_{d}, m_{d}, m_{u}, m_{d}\right)=s^{E ; \operatorname{reg}}\left(s, M_{Z},\{0\}_{4}\right)+C_{z}^{d} \text {, } \\
& s^{E ; \operatorname{fin}}\left(s, M_{Z}, m_{d}, m_{d}, m_{d}, m_{d}\right)=s^{E ; \operatorname{reg}}\left(s, M_{Z},\{0\}_{4}\right)+C_{Z}^{d} \text {, } \\
& s^{E ; \operatorname{spec}}\left(s, m_{u}, m_{u}, 0, M_{W}, 0\right)=s^{E ; \operatorname{spec} R}\left(s,\{0\}_{3}, M_{W}, 0\right)+\frac{L_{u}+L_{u}^{2}}{x_{W}-1}, \\
& s^{E ; \operatorname{spec}}\left(s, m_{d}, m_{d}, 0, M_{W}, 0\right)=s^{E ; \operatorname{spec} R}\left(s,\{0\}_{3}, M_{W}, 0\right)+\frac{L_{d}+L_{d}^{2}}{x_{W}-1} . \\
& s^{E ; \operatorname{fin}}\left(s, M_{W}, m_{t}, m_{b}, m_{t}, m_{b}\right)=s^{E ; \operatorname{reg}}\left(s, M_{W}, m_{t}, 0, m_{t}, 0\right) \\
& +\frac{L_{b}}{z_{t}-1}\left[1-\frac{1}{2} L_{b}-\frac{1}{x_{t}-1}\left(x_{t} L_{t}-L_{W}\right)\right], \\
& s^{E ; \operatorname{fin}}\left(s, M_{W}, m_{t}, m_{b}, m_{b}, m_{b}\right)=s^{E ; \operatorname{reg}}\left(s, M_{W}, m_{t},\{0\}_{3}\right)-L_{b}\left(1-L_{W}-\frac{1}{2} L_{b}\right)+\frac{z_{t}}{z_{t}-1} L_{b} L_{t} \text {, } \\
& s^{E ; \text { fin }}\left(s, M_{Z}, m_{b}, m_{b}, m_{b}, m_{b}\right)=s^{E ; \operatorname{reg}}\left(s, M_{Z},\{0\}_{4}\right)-L_{b}\left(1-L_{Z}-\frac{1}{2} L_{b}\right), \\
& s^{E ; \text { fin }}\left(s, M_{Z}, m_{b}, m_{b}, m_{t}, m_{b}\right)=s^{E ; \operatorname{reg}}\left(s, M_{Z},\{0\}_{2}, m_{t}, 0\right)+\frac{1}{z_{t}-1} L_{b}\left(1-L_{Z}-\frac{1}{2} L_{b}\right) \text {, } \\
& s^{E ; \operatorname{spec}}\left(s, m_{b}, m_{b}, 0, M_{W}, 0\right)=s^{E ; \operatorname{spec} R}\left(s,\{0\}_{3}, M_{W}, 0\right)+\frac{L_{b}+L_{b}^{2}}{z_{t}-1} .
\end{aligned}
$$




\section{The limit $p^{2} \rightarrow 0$}

There are several places where one needs Green functions, self-energies in particular, evaluated at $p^{2}=0$; e.g. renormalization of the electric charge requires the vacuum polarization function subtracted at $p^{2}=0$ and corrections to $G_{F}$ require the $W-W$ self-energy in the same limit.

Of course, evaluation of two-loop two-point functions at $p^{2}=0$ follows a slightly different and somehow independent approach. Every two-loop two-point diagram at $p^{2}=0$ is a vacuum bubble that can be reduced to one master integral by means of integration-by-part identities. However, we have opted for a different approach where we start from the result at arbitrary $p^{2}$ and perform the limit $p^{2} \rightarrow 0$; in this way we have a powerful check on the correctness of the original procedure.

\section{D.1 One-loop functions}

For most of the functions appearing in an arbitrary self-energy the limit $s=-p^{2} \rightarrow 0$ is almost trivial; nevertheless we report the corresponding expansions:

$$
\beta^{-1}\left(z_{m}\right) L_{\beta}\left(z_{m}\right)=-\frac{1}{2} z_{m}^{-1}-\frac{1}{12} z_{m}^{-2}-\frac{1}{60} z_{m}^{-3},
$$

with $z_{m}=m^{2} / s$ and $L_{m}=\ln z_{m}$.

$$
b_{0}^{\epsilon}\left(2,1, s, m^{2}, m^{2}\right)=z_{m}^{-1}\left[L_{m}+\frac{1}{6} z_{m}^{-1}\left(L_{m}-1\right)+\frac{1}{30} z_{m}^{-2}\left(L_{m}-\frac{3}{2}\right)\right],
$$

where $L_{m}=\ln z_{m}$. Similarly we obtain

$$
\begin{aligned}
b_{0}^{\epsilon}\left(1,1, s, m^{2}, m^{2}\right) & =-L_{m}^{2}+\frac{1}{3} z_{m}^{-1} L_{m}+\frac{1}{30} z_{m}^{-2}\left(L_{m}-1\right)+\frac{1}{210} z_{m}^{-3}\left(L_{m}-\frac{21}{14}\right), \\
b_{0}^{\epsilon}\left(1,1, s, 0, m^{2}\right) & =-2+2 L_{m}-L_{m}^{2}-z_{m}^{-1}\left(\frac{3}{2}-L_{m}\right)-z_{m}^{-2}\left(\frac{17}{18}-\frac{1}{3} L_{m}\right), \\
b_{0}^{\epsilon}\left(2,1, s, m^{2}, 0\right) & =2 z_{m}^{-1}\left(L_{m}-1\right)+z_{m}^{-2}\left(L_{m}-\frac{5}{2}\right) .
\end{aligned}
$$

The expansions for $b_{0}^{\epsilon}$ with unequal masses are rather long and will not be reported here.

\section{D.2 Two-loop functions}

For two-loop functions we rewrite scaled functions, e.g. $s^{A}$; fin in terms of the original ones, e.g. $S^{A}$; when a Taylor expansion is needed we derive it in terms of vacuum bubbles. Consider an example; given

$$
\begin{aligned}
s^{A ; \text { fin }}\left(s, m_{1}, m_{2}, m_{3}\right) & =s^{-1}\left[L_{W}\left(\frac{s}{2}-3 \sum_{i} m_{i}^{2}\right)+2 L_{W} \sum_{i} m_{i}^{2} L_{i}-L_{W}^{2} \sum_{i} m_{i}^{2}\right. \\
& \left.+\mathcal{P}_{U V} S^{A}\left(s, m_{1}, m_{2}, m_{3}\right)\right],
\end{aligned}
$$

where the operator $P_{U V}$ acts as

$$
\mathcal{P}_{U V} \epsilon^{-n}=0, \quad \mathcal{P}_{U V} \Delta_{U V}=0 .
$$

we use the first few terms in the expansion, e.g.

$$
\begin{aligned}
S^{A}\left(s, m_{1}, m_{2}, m_{3}\right) & =T_{111}\left(m_{1}, m_{3}, m_{2}\right)+\left[T_{121}\left(m_{1}, m_{3}, m_{2}\right)-\frac{4}{n} T_{121}\left(m_{1}, m_{3}, m_{2}\right)\right. \\
& \left.+\frac{4}{n} m_{3}^{2} T_{131}\left(m_{1}, m_{3}, m_{2}\right)\right] s+\mathcal{O}\left(s^{2}\right)
\end{aligned}
$$

IBP techniques are then use to reduce vacuum bubbles to our master integral $T_{121}\left(m_{1}, m_{3}, m_{2}\right)$. Therefore, one function suffices in deriving the final result. This sort of results is relevant whenewer we need corrections 
to the limit $s \rightarrow 0$ or when we want to prove that vacuum bubbles give the correct limit of the theory. For tensor integrals some additional work is needed; first we give an example,

$$
\int d^{n} q_{1} d^{n} q_{2} \frac{q_{i}^{\mu} q_{j}^{\nu}}{\left(q_{1}^{2}+m_{1}^{2}\right)^{n_{1}}\left(q_{2}^{2}+m_{3}^{2}\right)^{n_{2}}\left(\left(q_{1}-q_{2}\right)^{2}+m_{2}^{2}\right)^{n_{3}}}=T_{n_{1}, n_{2}, n_{3}}^{i j}\left(m_{1}, m_{3}, m_{2}\right) \delta^{\mu \nu},
$$

and derive the reduction of vacuum bubble form factors in terms of scalar vacuum bubbles,

$$
\begin{aligned}
& n T_{n_{1}, n_{2}, n_{3}}^{11}=T_{n_{1}-1, n_{2}, n_{3}}-m_{1}^{2} T_{n_{1}, n_{2}, n_{3}}, \\
& n T_{n_{1}, n_{2}, n_{3}}^{12}=\frac{1}{2} T_{n_{1}-1, n_{2}, n_{3}}+\frac{1}{2} T_{n_{1}, n_{2}-1, n_{3}}-\frac{1}{2} T_{n_{1}, n_{2}, n_{3}-1}+\frac{1}{2}\left(m_{3}^{2}-m_{1}^{2}-m_{2}^{2}\right) T_{n_{1}, n_{2}, n_{3}}, \\
& n T_{n_{1}, n_{2}, n_{3}}^{22}=T_{n_{1}, n_{2}-1, n_{3}}-m_{2}^{2} T_{n_{1}, n_{2}, n_{3}},
\end{aligned}
$$

where all $T$ have argument $m_{1}, m_{2}, m_{3}$. Suppose now that we have to compute

$$
\begin{aligned}
S^{D}(\mu \mid 0) & =\int d^{n} q_{1} d^{n} q_{2} q_{1}^{\mu} \\
& \times\left[\left(q_{1}^{2}+m_{1}^{2}\right)\left(\left(q_{1}+p\right)^{2}+m_{1}^{2}\right)\left(\left(q_{1}-q_{2}\right)^{2}+m_{2}^{2}\right)\left(q_{2}^{2}+m_{3}^{2}\right)\left(\left(q_{2}+p\right)^{2}+m_{3}^{2}\right)\right]^{-1},
\end{aligned}
$$

in the limit $p^{2} \rightarrow 0$. the recipe is simple: expand the propagators, e.g.

$$
\left(\left(q_{1}+p\right)^{2}+m_{1}^{2}\right)^{-k}=\left(q_{1}^{2}+m_{1}^{2}\right)^{-k}-\left(p^{2}+2 p \cdot q_{1}\right)\left(q_{1}^{2}+m_{1}^{2}\right)^{-k-1}+\ldots,
$$

to obtain

$$
\begin{aligned}
n S_{11}^{D}(0,\{m\}) & =-T_{131}\left(m_{1}, m_{3}, m_{2}\right)-3 T_{221}\left(m_{1}, m_{3}, m_{2}\right) \\
& +\left(m_{1}^{2}-m_{2}^{2}+m_{3}^{2}\right) T_{231}\left(m_{1}, m_{3}, m_{2}\right)+2 m_{1}^{2} T_{321}\left(m_{1}, m_{3}, m_{2}\right)-A_{0}\left(2, m_{1}\right) A_{0}\left(3, m_{3}\right), \\
n S_{12}^{D}(0,\{m\}) & =-3 T_{221}\left(m_{1}, m_{3}, m_{2}\right)+2 m_{3}^{2} T_{231}\left(m_{1}, m_{3}, m_{2}\right) \\
& -T_{311}\left(m_{1}, m_{3}, m_{2}\right)+\left(m_{1}^{2}-m_{2}^{2}+m_{3}^{2}\right) T_{321}\left(m_{1}, m_{3}, m_{2}\right)-A_{0}\left(2, m_{3}\right) A_{0}\left(3, m_{1}\right) .
\end{aligned}
$$

For tensor integrals of higher rank we need to define the $T^{i j k l}$ form factors as

$$
\begin{aligned}
\int d^{n} q_{1} d^{n} q_{2} \frac{q_{i}^{\mu} q_{j}^{\nu} q_{k}^{\alpha} q_{l}^{\beta}}{\left(q_{1}^{2}+m_{1}^{2}\right)^{n_{1}}\left(q_{2}^{2}+m_{3}^{2}\right)^{n_{2}}\left(\left(q_{1}-q_{2}\right)^{2}+m_{2}^{2}\right)^{n_{3}}} & =T_{A ; n_{1}, n_{2}, n_{3}}^{i j k l}\left(m_{1}, m_{3}, m_{2}\right) \delta_{\mu \nu} \delta_{\alpha \beta} \\
& +T_{B ; n_{1}, n_{2}, n_{3}}^{i j k l}\left(m_{1}, m_{3}, m_{2}\right) \delta_{\mu \alpha} \delta_{\nu \beta} \\
& +T_{C ; n_{1}, n_{2}, n_{3}}^{i j k l}\left(m_{1}, m_{3}, m_{2}\right) \delta_{\mu \beta} \delta_{\nu \alpha}
\end{aligned}
$$

with the following results:

$$
\begin{gathered}
n^{2} T_{A ; n_{1}, n_{2}, n_{3}}^{1122}=-n T_{B ; n_{1}, n_{2}, n_{3}}^{1122}-n T_{C ; n_{1}, n_{2}, n_{3}}^{1122} \\
+T_{n_{1}-1, n_{2}-1, n_{3}}-m_{2}^{2} T_{n_{1}-1, n_{2}, n_{3}}-m_{1}^{2} T_{n_{1}, n_{2}-1, n_{3}}+m_{1}^{2} m_{2}^{2} T_{n_{1}, n_{2}, n_{3}} \\
(n-1) T_{C ; n_{1}, n_{2}, n_{3}}^{1122}=\left(1-n^{2}\right) T_{B ; n_{1}, n_{2}, n_{3}}^{1122}+\frac{1}{4} T_{n_{1}-2, n_{2}, n_{3}} \\
+\left(\frac{1}{2}-\frac{1}{n}\right) T_{n_{1}-1, n_{2}-1, n_{3}}-\frac{1}{2} T_{n_{1}-1, n_{2}, n_{3}-1}+\left[\left(\frac{1}{n}-\frac{1}{2}\right) m_{2}^{2}+\frac{1}{2}\left(m_{3}^{2}-m_{1}^{2}\right)\right] T_{n_{1}-1, n_{2}, n_{3}} \\
+\frac{1}{4} T_{n_{1}, n_{2}, n_{3}-2}+\frac{1}{2}\left(m_{1}^{2}+m_{2}^{2}-m_{3}^{2}\right) T_{n_{1}, n_{2}, n_{3}-1}+\left[\frac{n-1}{n} m_{1}^{2} m_{2}^{2}+\frac{1}{4} \lambda\left(m_{1}^{2}, m_{2}^{2}, m_{3}^{2}\right)\right] T_{n_{1}, n_{2}, n_{3}} \\
+\frac{1}{4} T_{n_{1}, n_{2}-2, n_{3}}-\frac{1}{2} T_{n_{1}, n_{2}-1, n_{3}-1}+\left[\left(\frac{1}{n}-\frac{1}{2}\right) m_{1}^{2}+\frac{1}{2}\left(m_{3}^{2}-m_{2}^{2}\right)\right] T_{n_{1}, n_{2}-1, n_{3}}
\end{gathered}
$$




$$
\begin{aligned}
& n(n-1)(n+2) T_{B ; n_{1}, n_{2}, n_{3}}^{1122}=\frac{1}{4} n T_{n_{1}-2, n_{2}, n_{3}}-\left(1-\frac{1}{2} n\right) T_{n_{1}-1, n_{2}-1, n_{3}} \\
& -\frac{1}{2} n T_{n_{1}-1, n_{2}, n_{3}-1}-\left[\left(\frac{1}{2} n-1\right) m_{2}^{2}+\frac{1}{2}\left(m_{1}^{2}-m_{3}^{2}\right)\right] T_{n_{1}-1, n_{2}, n_{3}}+\frac{1}{4} n T_{n_{1}, n_{2}-2, n_{3}}-\frac{1}{2} n T_{n_{1}, n_{2}-1, n_{3}-1} \\
& -\left[\left(\frac{1}{2} n-1\right) m_{1}^{2}+\frac{1}{2}\left(m_{2}^{2}-m_{3}^{2}\right)\right] T_{n_{1}, n_{2}-1, n_{3}}+\frac{1}{4} n T_{n_{1}, n_{2}, n_{3}-2}-\frac{1}{2} n\left(m_{3}^{2}-m_{1}^{2}-m_{2}^{2}\right) T_{n_{1}, n_{2}, n_{3}-1} \\
& -\left[(1-n) m_{1}^{2} m_{2}^{2}-\frac{1}{4} n \lambda\left(m_{1}^{2}, m_{2}^{2}, m_{3}^{2}\right)\right] T_{n_{1}, n_{2}, n_{3}} \text {, } \\
& n^{2} T_{A ; n_{1}, n_{2}, n_{3}}^{1222}=-n T_{B ; n_{1}, n_{2}, n_{3}}^{1222}-n T_{C ; n_{1}, n_{2}, n_{3}}^{1222} \\
& +\frac{1}{2} T_{n_{1}-1, n_{2}-1, n_{3}}-\frac{1}{2} m_{2}^{2} T_{n_{1}-1, n_{2}, n_{3}}+\frac{1}{2} T_{n_{1}, n_{2}-2, n_{3}}-\frac{1}{2} T_{n_{1}, n_{2}-1, n_{3}-1} \\
& +\frac{1}{2}\left(m_{3}^{2}-2 m_{2}^{2}-m_{1}^{2}\right) T_{n_{1}, n_{2}-1, n_{3}}+\frac{1}{2} m_{2}^{2} T_{n_{1}, n_{2}, n_{3}-1}+\frac{1}{2} m_{2}^{2}\left(m_{1}^{2}+m_{2}^{2}-m_{3}^{2}\right) T_{n_{1}, n_{2}, n_{3}} \\
& T_{C ; n_{1}, n_{2}, n_{3}}^{1222}=-(n+1) T_{B ; n_{1}, n_{2}, n_{3}}^{1222}+\frac{1}{2 n} T_{n_{1}-1, n_{2}-1, n_{3}} \\
& -\frac{1}{2 n} m_{2}^{2} T_{n_{1}-1, n_{2}, n_{3}}+\frac{1}{2 n} T_{n_{1}, n_{2}-2, n_{3}}-\frac{1}{2 n} T_{n_{1}, n_{2}-1, n_{3}-1} \\
& +\frac{1}{n}\left[\frac{1}{2}\left(m_{3}^{2}-m_{1}^{2}\right)-m_{2}^{2}\right] T_{n_{1}, n_{2}-1, n_{3}} \\
& +\frac{1}{2 n} m_{2}^{2} T_{n_{1}, n_{2}, n_{3}-1}-\frac{1}{2 n} m_{2}^{2}\left(m_{3}^{2}-m_{1}^{2}-m_{2}^{2}\right) T_{n_{1}, n_{2}, n_{3}}, \\
& n(n+2) T_{B ; n_{1}, n_{2}, n_{3}}^{1222}=\frac{1}{2} T_{n_{1}-1, n_{2}-1, n_{3}}-\frac{1}{2} m_{2}^{2} T_{n_{1}-1, n_{2}, n_{3}} \\
& +\frac{1}{2} T_{n_{1}, n_{2}-2, n_{3}}-\frac{1}{2} T_{n_{1}, n_{2}-1, n_{3}-1}-\left[\frac{1}{2} m_{1}^{2}+m_{2}^{2}-\frac{1}{2} m_{3}^{2}\right] T_{n_{1}, n_{2}-1, n_{3}} \\
& +\frac{1}{2} m_{2}^{2} T_{n_{1}, n_{2}, n_{3}-1}-\frac{1}{2} m_{2}^{2}\left(m_{3}^{2}-m_{1}^{2}-m_{2}^{2}\right) T_{n_{1}, n_{2}, n_{3}}, \\
& n^{2} T_{A ; n_{1}, n_{2}, n_{3}}^{2222}=-n T_{B ; n_{1}, n_{2}, n_{3}}^{2222}-n T_{C ; n_{1}, n_{2}, n_{3}}^{2222} \\
& +T_{n_{1}, n_{2}-2, n_{3}}-2 m_{2}^{2} T_{n_{1}, n_{2}-1, n_{3}}+m_{2}^{4} T_{n_{1}, n_{2}, n_{3}}, \\
& T_{C ; n_{1}, n_{2}, n_{3}}^{2222}=-(n+1) T_{B ; n_{1}, n_{2}, n_{3}}^{2222}+\frac{1}{n} T_{n_{1}, n_{2}-2, n_{3}} \\
& -2 \frac{1}{n} m_{2}^{2} T_{n_{1}, n_{2}-1, n_{3}}+\frac{1}{n} m_{2}^{4} T_{n_{1}, n_{2}, n_{3}}, \\
& n(n+2) T_{B ; n_{1}, n_{2}, n_{3}}^{2222}=T_{n_{1}, n_{2}-2, n_{3}}-2 m_{2}^{2} T_{n_{1}, n_{2}-1, n_{3}}+m_{2}^{4} T_{n_{1}, n_{2}, n_{3}} .
\end{aligned}
$$

For instance, we obtain

$$
S_{221}^{E}(0,\{m\})=4\left(\frac{1}{n}-\frac{1}{n+2}\right)\left[T_{131}-2 m_{3}^{2} T_{141}+m_{3}^{4} T_{151}\right] .
$$

\section{- Laurent expansion}

There are cases, however, where a Taylor expansion is not allowed. Consider $S^{C}\left(s, m_{1}, m_{2},\{0\}_{2}\right)$ which contains propagator factors $q_{2}^{2}$ and $\left(q_{2}+p\right)^{2}$. The limit $p^{2} \rightarrow 0$ is IR divergent and the corresponding 
singularity should not be regularized by $\epsilon$, a fact that would change the pole structure of the diagram, but a Laurent expansion around $p^{2}=0$ is needed. In the following list, where we define

$$
L_{W}=\ln \frac{M_{W}^{2}}{s}, \quad \mathcal{L}_{m}=\ln \frac{m^{2}}{M_{W}^{2}}, \quad \mathcal{L}_{m_{i}}=\ln \frac{m_{i}^{2}}{M_{W}^{2}},
$$

and where $L_{\text {an }}$ is given in Eq.(21), we present the relevant limits:

$$
\begin{aligned}
& S^{C} ; \text { fin }\left(s, 0, m,\{0\}_{2}\right)=L_{W} L_{\mathrm{an}}-\left(L_{W}+L_{\mathrm{an}}\right)\left(\frac{23}{6}-\mathcal{L}_{m}\right)-\frac{1}{2} L_{W}^{2}-\frac{107}{12}+\frac{9}{2} \zeta(2) \\
& +2 \mathcal{L}_{m}+\frac{1}{2} \mathcal{L}_{m}^{2}+\mathcal{O}(s) \\
& S^{C} ; \operatorname{fin}\left(s, m_{1}, m_{2},\{0\}_{2}\right)=L_{W} L_{\text {an }}-L_{W}\left[\frac{23}{6}-\mathcal{L}_{m_{1}}-\left(\mathcal{L}_{m_{1}}+\mathcal{L}_{m_{2}}\right) \frac{x_{2}}{x_{1}-x_{2}}\right]-\frac{1}{2} L_{W}^{2} \\
& +L_{\text {an }}\left[\frac{23}{6}-\mathcal{L}_{m_{1}}-\left(\mathcal{L}_{m_{1}}-\mathcal{L}_{m_{2}}\right) \frac{x_{2}}{x_{1}-x_{2}}\right]-\frac{113}{12}+3 \zeta(2)+3 \mathcal{L}_{m_{1}} \\
& -\frac{1}{2} \mathcal{L}_{m_{1}}^{2}-T_{121}^{\mathrm{fin}}\left(0, m_{1}, m_{2}\right)+\frac{x_{2}}{x_{1}-x_{2}}\left[-\mathcal{L}_{m_{1}} \mathcal{L}_{m_{2}}+4 \mathcal{L}_{m_{1}}-2 \mathcal{L}_{m_{2}}-\mathcal{L}_{m_{1}}^{2}\right. \\
& \left.-\zeta(2)-1-2 T_{121}^{\mathrm{fin}}\left(0, m_{1}, m_{2}\right)\right]+\mathcal{O}(s) \text {. }
\end{aligned}
$$

Note that these functions are of $\mathcal{O}\left(\ln ^{2} s\right)$ for $s \rightarrow 0$. Furthermore,

$$
\begin{aligned}
s S^{E ; \text { fin }}\left(s, 0, m,\{0\}_{3}\right) & =L_{W} L_{\text {an }}-\left(L_{W}-L_{\text {an }}\right)\left(\frac{7}{3}-L_{W 2}\right)-\frac{1}{2}\left(L_{W}^{2}+L_{\text {an }}^{2}+\mathcal{L}_{m}^{2}\right)+\frac{11}{6} \mathcal{L}_{m}-\frac{49}{12}, \\
s S^{E ; \text { fin }}\left(s, m, m,\{0\}_{3}\right) & =L_{W} L_{\text {an }}-\left(L_{W}-L_{\text {an }}\right)\left(\frac{4}{3}-\mathcal{L}_{m}\right)-\frac{1}{2}\left(L_{W}^{2}+L_{\text {an }}^{2}+\mathcal{L}_{m}^{2}\right)+\frac{5}{6} \mathcal{L}_{m}-\frac{9}{4}, \\
s S^{E ; \text { fin }}\left(s, m_{1}, m_{2},\{0\}_{3}\right) & =L_{W} L_{\text {an }}-\left(L_{W}-L_{\text {an }}\right)\left(\frac{7}{3}-\mathcal{L}_{m_{1}}\right)+\frac{x_{2}}{x_{1}-x_{2}}\left(\mathcal{L}_{m_{1}}-\mathcal{L}_{m_{2}}\right) \\
& -\frac{1}{2}\left(L_{W}^{2}+L_{\text {an }}^{2}+\mathcal{L}_{m_{1}}^{2}\right)-\frac{49}{12}+\frac{11}{6} \mathcal{L}_{m_{1}} \\
& +\frac{x_{2}}{x_{1}-x_{2}}\left[\frac{11}{6}\left(\mathcal{L}_{m_{1}}-\mathcal{L}_{m_{2}}\right)-\frac{1}{2}\left(\mathcal{L}_{m_{1}}^{2}-\mathcal{L}_{m_{2}}^{2}\right)\right] .
\end{aligned}
$$

\section{D.3 Limit $m_{f} \rightarrow 0$ of $p^{2} \rightarrow 0$}

After performing the limit $p^{2} \rightarrow 0$ we still would like to set light fermion masses to zero, whenever possible. Such is the situation for our main traget, the inclusion of $\alpha$ and of $G_{F}$ in the IPS. Functions for which a Laurent expansion is needed include all cases where we have a vacuum bubble $T_{n_{1} n_{2} n_{3}}(0, m, M)$, or permutations of it, and $n_{1} \geq 2$. For expanding these functions we have developed a special technique, based on the idea of writing differential equations for diagrams [24. This technique works in all cases where we know how to compute integration constants.

- $\underline{T_{121}(m, M, m) \text { with } m \rightarrow 0}$

Consider the following example: $T_{121}(m, M, m)$ with $m \rightarrow 0$. We write the equation

$$
\frac{d}{d m^{2}} T_{121}(m, M, m)=-T_{221}(m, M, m)-T_{122}(m, M, m) .
$$

We use IBP identities to express the r.h.s of Eq.(272) in terms of $T_{121}(m, M, m)$. Using Eq.(31) we make the ansätze,

$$
T_{f}(m, M, m)=\sum_{n=0} a_{n}\left(\frac{m^{2}}{M^{2}}\right)^{n}+\mathcal{L}_{m} \sum_{n=0} b_{n}\left(\frac{m^{2}}{M^{2}}\right)^{n}+\mathcal{L}_{m}^{2} \sum_{n=0} c_{n}\left(\frac{m^{2}}{M^{2}}\right)^{n}
$$


insert into Eq.(272) and derive

$$
a_{0}=T_{f}(0, M, 0)=-\frac{1}{2}-\frac{3}{2} \zeta(2)+\mathcal{L}_{M}-\mathcal{L}_{M}^{2},
$$

where $\mathcal{L}_{m}=\ln m^{2} / M_{W}^{2}$ and $\mathcal{L}_{M}=\ln M^{2} / M_{W}^{2}$. Here, the integration constant is known, see Eq.(242). Furthermore, we have

$$
\begin{aligned}
& a_{1}=2+2 \mathcal{L}_{M}, \quad a_{2}=-\frac{1}{2}\left[-2 \zeta(2)-5-2 \mathcal{L}_{M}-2 \mathcal{L}_{M}^{2}-4 a_{0}\right], \\
& a_{3}=-\frac{1}{3}\left[-12 \zeta(2)-\frac{92}{3}-8 \mathcal{L}_{M}-12 \mathcal{L}_{M}^{2}-24 a_{0}\right] \\
& b_{0}=0, \quad b_{1}=-2, \quad b_{2}=-3+2 \mathcal{L}_{M}, \quad b_{3}=-\frac{32}{3}+8 \mathcal{L}_{M}, \\
& c_{0}=0, \quad c_{1}=0, \quad c_{2}=-1, \quad c_{3}=-4,
\end{aligned}
$$

etc.

$\underline{T_{f}(m, M, 0) \text { with } m \rightarrow 0}$

Similarly to the previous case, we obtain

$$
T_{f}(m, M, 0)=\sum_{n=0} a_{n}\left(\frac{m^{2}}{M^{2}}\right)^{n}+\ln \frac{m^{2}}{M_{W}^{2}} \sum_{n=0} b_{n}\left(\frac{m^{2}}{M^{2}}\right)^{n},
$$

with coefficients $a_{0}=T_{f}(0, M, 0)$ again given in Eq.(242) and

$$
\begin{aligned}
& a_{1}=1+\mathcal{L}_{M}, \quad a_{2}=\frac{1}{2}\left(\frac{1}{2}+\mathcal{L}_{M}\right), \quad a_{3}=\frac{1}{3}\left(\frac{1}{3}+\mathcal{L}_{M}\right), \\
& b 0=0, \quad b_{1}=-1, \quad b_{2}=-\frac{1}{2}, \quad b_{3}=-\frac{1}{3} .
\end{aligned}
$$

- small, unequal, masses

For small, unequal, masses we write two differential equations,

$$
\frac{d}{d m_{1}^{2}} T_{121}\left(m_{1}, M, m_{2}\right)=-T_{221}\left(m_{1}, M, m_{2}\right), \quad \frac{d}{d m_{2}^{2}} T_{121}\left(m_{1}, M, m_{2}\right)=-T_{122}\left(m_{1}, M, m_{2}\right),
$$

and introduce the ansatz

$$
\begin{aligned}
T_{f}\left(m_{1}, M, m_{2}\right) & =a_{0}+a_{1} \frac{m_{1}^{2}+m_{2}^{2}}{M^{2}}+a_{2} \frac{m_{1}^{4}+m_{2}^{4}}{M^{4}}+a_{3} \frac{m_{1}^{2} m_{2}^{2}}{M^{4}}+a_{4} \frac{m_{1}^{6}+m_{2}^{6}}{M^{6}}+a_{5} m_{1}^{2} m_{2}^{2} \frac{m_{1}^{2}+m_{2}^{2}}{M^{6}} \\
& +\ln \frac{m_{1}^{2}}{M_{W}^{2}}\left[b_{1} \frac{m_{1}^{2}}{M^{2}}+b_{2} \frac{m_{1}^{2} m_{2}^{2}}{M^{4}}+b_{3} \frac{m_{1}^{4}}{M^{4}}+b_{4} \frac{m_{1}^{2} m_{2}^{4}}{M^{6}}+b_{5} \frac{m_{1}^{4} m_{2}^{2}}{M^{6}}+b_{6} \frac{m_{1}^{6}}{M^{6}}\right] \\
& +\ln \frac{m_{2}^{2}}{M_{W}^{2}}\left[b_{1} \frac{m_{2}^{2}}{M^{2}}+b_{2} \frac{m_{2}^{2} m_{1}^{2}}{M^{4}}+b_{3} \frac{m_{2}^{4}}{M^{4}}+b_{4} \frac{m_{2}^{2} m_{1}^{4}}{M^{6}}+b_{5} \frac{m_{2}^{4} m_{1}^{2}}{M^{6}}+b_{6} \frac{m_{2}^{6}}{M^{6}}\right] \\
& +\ln \frac{m_{1}^{2}}{M_{W}^{2}} \ln \frac{m_{2}^{2}}{M_{W}^{2}}\left[c_{1} \frac{m_{1}^{2} m_{2}^{2}}{M^{4}}+c_{2} m_{1}^{2} m_{2}^{2} \frac{m_{1}^{2}+m_{2}^{2}}{M^{6}}\right] .
\end{aligned}
$$

The solution is $a_{0}=T_{f}(0, M, 0)$ (Eq.(242) $)$ and

$$
\begin{aligned}
& a_{1}=1+\mathcal{L}_{M}, \quad a_{2}=\frac{1}{2}\left(\frac{1}{2}+\mathcal{L}_{M}\right), \quad a_{3}=2+\zeta(2)+\mathcal{L}_{M}^{2}+2 a_{0}, \\
& a_{4}=\frac{1}{3}\left(\frac{1}{3}+\mathcal{L}_{M}\right), \quad a_{5}=5+2 \zeta(2)+\mathcal{L}_{M}+2 \mathcal{L}_{M}^{2}+4 a_{0}, \\
& b_{1}=-1, \quad b_{2}=-1+\mathcal{L}_{M}, \quad b_{3}=-\frac{1}{2}, \quad b_{4}=-1+2 \mathcal{L}_{M}, \quad b_{5}=-4+2 \mathcal{L}_{M}, \quad b_{6}=-\frac{1}{3}, \\
& c_{1}=-1, \quad c_{2}=-2 .
\end{aligned}
$$




\section{- one small mass}

For one small mass, $m \ll m_{1}, m_{2}$, we obtain

$$
T_{f}\left(m, m_{1}, m_{2}\right)=\sum_{n=0} a_{n}\left(\frac{m^{2}}{M_{W}^{2}}\right)^{n}+\mathcal{L}_{m} \sum_{n=1} b_{n}\left(\frac{m^{2}}{M_{W}^{2}}\right)^{n},
$$

with a solution $a_{0}=T_{f}\left(0, m_{1}, m_{2}\right)$ and

$$
\begin{aligned}
a_{1} & =\mathcal{L}_{1} \mathcal{L}_{2} \frac{x_{2}}{X^{2}}+\mathcal{L}_{1}\left(-\frac{x_{2}}{X^{2}}+\frac{1}{X}\right)+\mathcal{L}_{1}^{2} \frac{x_{2}}{X^{2}}-\mathcal{L}_{2} \frac{x_{2}}{X^{2}}+\zeta(2) \frac{x_{2}}{X^{2}}+\frac{x_{2}}{X^{2}}+\frac{1}{X}+2 a_{0} \frac{x_{2}}{X^{2}}, \\
b_{1} & =\mathcal{L}_{1} \frac{x_{2}}{X^{2}}-\mathcal{L}_{2} \frac{x_{2}}{X^{2}}-\frac{1}{X} \\
a_{2} & =\mathcal{L}_{1} \mathcal{L}_{2}\left(3 \frac{x_{2}^{2}}{X^{4}}+2 \frac{x_{2}}{X^{3}}\right)+\mathcal{L}_{1}\left(-\frac{5}{2} \frac{x_{2}^{2}}{X^{4}}+\frac{1}{2} \frac{1}{X^{2}}\right)+\mathcal{L}_{1}^{2}\left(3 \frac{x_{2}^{2}}{X^{4}}+2 \frac{x_{2}}{X^{3}}\right) \\
& +\mathcal{L}_{2}\left(-\frac{7}{2} \frac{x_{2}^{2}}{X^{4}}-\frac{x_{2}}{X^{3}}\right)+3 \zeta(2) \frac{x_{2}^{2}}{X^{4}}+2 \zeta(2) \frac{x_{2}}{X^{3}}+3 \frac{x_{2}^{2}}{X^{4}}+\frac{9}{2} \frac{x_{2}}{X^{3}}+\frac{1}{4} \frac{1}{X^{2}}+a_{0}\left(6 \frac{x_{2}^{2}}{X^{4}}+4 \frac{x_{2}}{X^{3}}\right), \\
b_{2} & =\mathcal{L}_{1}\left(3 \frac{x_{2}^{2}}{X^{4}}+2 \frac{x_{2}}{X^{3}}\right)+\mathcal{L}_{2}\left(-3 \frac{x_{2}^{2}}{X^{4}}-2 \frac{x_{2}}{X^{3}}\right)-3 \frac{x_{2}}{X^{3}}-\frac{1}{2} \frac{1}{X^{2}}, \\
a_{3} & =\mathcal{L}_{1} \mathcal{L}_{2}\left(10 \frac{x_{2}^{3}}{X^{6}}+12 \frac{x_{2}^{2}}{X^{5}}+3 \frac{x_{2}}{X^{4}}\right)+\mathcal{L}_{1}\left(-\frac{23}{3} \frac{x_{2}^{3}}{X^{6}}-4 \frac{x_{2}^{2}}{X^{5}}+2 \frac{x_{2}}{X^{4}}+\frac{1}{3} \frac{1}{X^{3}}\right) \\
& +\mathcal{L}_{1}^{2}\left(10 \frac{x_{2}^{3}}{X^{6}}+12 \frac{x_{2}^{2}}{X^{5}}+3 \frac{x_{2}}{X^{4}}\right)+\mathcal{L}_{2}\left(-\frac{37}{3} \frac{x_{2}^{3}}{X^{6}}-10 \frac{x_{2}^{2}}{X^{5}}-\frac{x_{2}}{X^{4}}\right) \\
& +10 \zeta(2) \frac{x_{2}^{3}}{X^{6}}+12 \zeta(2) \frac{x_{2}^{2}}{X^{5}}+3 \zeta(2) \frac{x_{2}}{X^{4}}+10 \frac{x_{2}^{3}}{X^{6}}+\frac{59}{3} \frac{x_{2}^{2}}{X^{5}}+\frac{49}{6} \frac{x_{2}}{X^{4}}+\frac{1}{9} \frac{1}{X^{3}} \\
& +a_{0}\left(20 \frac{x_{2}^{3}}{X^{6}}+24 \frac{x_{2}^{2}}{X^{5}}+6 \frac{x_{2}}{X^{4}}\right), \\
b_{3} & =\mathcal{L}_{1}\left(10 \frac{x_{2}^{3}}{X^{6}}+12 \frac{x_{2}^{2}}{X^{5}}+3 \frac{x_{2}}{X^{4}}\right)+\mathcal{L}_{2}\left(-10 \frac{x_{2}^{3}}{X^{6}}-12 \frac{x_{2}^{2}}{X^{5}}-3 \frac{x_{2}}{X^{4}}\right)-10 \frac{x_{2}^{2}}{X^{5}}-7 \frac{x_{2}}{X^{4}}-\frac{1}{3} \frac{1}{X^{3}}, \quad(282
\end{aligned}
$$

where we have introduced

$$
x_{i}=\frac{m_{i}^{2}}{M_{W}^{2}}, \quad X=x_{1}-x_{2}, \quad \mathcal{L}_{i}=\ln x_{i}, \quad \mathcal{L}_{m}=\ln \frac{m^{2}}{M_{W}^{2}} .
$$

\section{E Finite mass renormalization in $\Pi^{R}$}

As we have discussed in the previous sections there is full control upon finite parts at arbitrary scale. In the following $M_{0}=M / c_{\theta}, M$ being the renormalized $W$ boson mass; furthermore, $M_{H}$ (etc) is the $H$ boson (etc) renormalized mass. Consider, for example, the subtracted two-loop photon self-energy, as defined in sect. 6 of I,

$$
\Pi^{R}(s)=\Pi_{Q Q ; \operatorname{ext}}^{(2)}(s)-\Pi_{Q Q ; \operatorname{ext}}^{(2)}(0)=\frac{1}{c_{\theta}^{2}} F_{1}^{A A}(s)+F_{2}^{A A}(s)+s_{\theta}^{2} F_{3}^{A A}(s) .
$$

Before using $\Pi^{R}(s)$ for physical predictions all renormalized masses must be replaced by on-shell masses, using $M_{R}=M_{O S}+\mathcal{O}\left(g^{2}\right)$ in one-loop terms and $M_{R}=M_{O S}$ in two-loop terms (see Section [5.3, Eqs. (100), (96) and (104)). For instance, the effect of finite mass renormalization on $\Pi(s)=\Pi^{R}(s)-\Pi^{R}(0)$ is as follows; define UV finite factors

$$
M^{2}=M_{W}^{2}\left(1+\frac{g^{2}}{\pi^{2}} \delta M^{2}\right)
$$


etc. In the massless limit for fermions we get

$$
\begin{gathered}
\Pi^{(1)}(s) \rightarrow \Pi^{(1)}(s)+\frac{g^{4}}{\pi^{4}} s_{\theta}^{2} \Delta \Pi(s), \\
\Delta \Pi(s)=\frac{1}{12} \sum_{l} \delta m_{l}^{2}+\frac{1}{8} x_{W}\left(1+12 x_{W}\right) \delta M^{2} b_{0}^{\mathrm{fin}}\left(2,1, s, M_{W}, M_{W}\right) \\
-\frac{4}{3} x_{T}^{2} \Delta m_{t}^{2} b_{0}^{\mathrm{fin}}\left(2,1, s, m_{t}, m_{t}\right)-\frac{3}{4}\left(x_{W}+\frac{1}{4}\right) \delta M^{2}+\frac{2}{3}\left(x_{T}+\frac{1}{6}\right) \delta m_{t}^{2},
\end{gathered}
$$

with $x_{i}=m_{i}^{2} / s$. The complete expression for $\Pi(s)$ is too long to be reported here and can be found at http://www.to.infn.it/ giampier/REN/pis.ps. 


\section{References}

[1] G. 't Hooft and M. J. G. Veltman, Nucl. Phys. B 44, 189 (1972);

G. 't Hooft and M. J. G. Veltman, Nucl. Phys. B 50, 318 (1972).

[2] S. Actis, A. Ferroglia, M. Passera and G. Passarino. "Two-Loop Renormalization of the Standard Model. Part I: Prolegomena" .

[3] A. Denner, G. Weiglein and S. Dittmaier, Nucl. Phys. B 440 (1995) 95 [arXiv hep-ph/9410338.

[4] F. Jegerlehner and J. Fleischer, Phys. Lett. B 151 (1985) 65;

F. Jegerlehner and J. Fleischer, Acta Phys. Polon. B 17 (1986) 709.

[5] J. C. Taylor, Nucl. Phys. B 33 (1971) 436;

A. A. Slavnov, Theor. Math. Phys. 10 (1972) 99 [Teor. Mat. Fiz. 10 (1972) 153];

M. J. G. Veltman, Nucl. Phys. B 21 (1970) 288;

Y. Takahashi, Nuovo Cim. 6 (1957) 371.

[6] A. Freitas, W. Hollik, W. Walter and G. Weiglein, Nucl. Phys. B 632 (2002) 189, [Erratum, ibidem B 666 (2003) 305], hep-ph/0202131;

A. Freitas, W. Hollik, W. Walter and G. Weiglein, Phys. Lett. B 495 (2000) 338 [Erratum-ibid. B 570 (2003) 260] [arXiv:hep-ph/0007091;

M. Awramik, M. Czakon, A. Freitas and G. Weiglein, Phys. Rev. D 69 (2004) 053006 [arXiv:hep-ph/0311148];

M. Awramik and M. Czakon, Phys. Lett. B 568 (2003) 48 [arXiv:hep-ph/0305248;

M. Awramik, M. Czakon, A. Onishchenko and O. Veretin, Phys. Rev. D 68 (2003) 053004 [arXiv:hep-ph/0209084];

G. Degrassi and A. Vicini, Phys. Rev. D 69 (2004) 073007 [arXiv:hep-ph/0307122].

[7] J. A. M. Vermaseren, [arXiv math-ph/0010025].

[8] S. Actis, A. Ferroglia, G. Passarino, M. Passera, C. Sturm and S. Uccirati, GraphShot, a FORM package for automatic generation and manipulation of one- and two-loop Feynman diagrams, unpublished.

[9] G. Passarino and S. Uccirati, LoopBack, a FORTRAN/95 code for numerical evaluation of one- and two-loop Feynman diagrams, unpublished.

[10] G. Passarino, Nucl. Phys. B 619 (2001) 257 [arXiv hep-ph/0108252].

[11] G. Passarino and S. Uccirati, Nucl. Phys. B 629 (2002) 97 [arXiv hep-ph/0112004].

[12] A. Ferroglia, M. Passera, G. Passarino and S. Uccirati, Nucl. Phys. B 680 (2004) 199 [arXiv:hep-ph/0311186].

[13] G. Passarino and S. Uccirati, Nucl. Phys. B 747 (2006) 113 [arXiv hep-ph/0603121].

[14] S. Actis, A. Ferroglia, G. Passarino, M. Passera and S. Uccirati, Nucl. Phys. B 703 (2004) 3 [arXiv:hep-ph/0402132].

[15] A. Ferroglia, M. Passera, G. Passarino and S. Uccirati, Nucl. Phys. B 650 (2003) 162 [arXiv:hep-ph/0209219].

[16] A. Denner, B. Jantzen and S. Pozzorini, arXiv hep-ph/0608326

S. Dittmaier, Nucl. Phys. B 675 (2003) 447 [arXiv'hep-ph/0308246.

[17] S. Actis, G. Passarino and S. Uccirati, Nucl. Phys. Proc. Suppl. 160 (2006) 145 [arXiv:hep-ph/0608294].

[18] G. Passarino and M. J. G. Veltman, Nucl. Phys. B 160 (1979) 151. 
[19] G. Weiglein, R. Scharf and M. Bohm, Nucl. Phys. B 416, 606 (1994) [arXiv hep-ph/9310358].

[20] F. V. Tkachov, Phys. Lett. B 100 (1981) 65;

F. V. Tkachov, New methods for evaluation of multi-loop Feynman diagrams, PhD thesis, INR, Moscow, March 1984;

K. G. Chetyrkin and F. V. Tkachov, Nucl. Phys. B 192 (1981) 159;

G. 't Hooft and M. Veltman, Nucl. Phys. B 44 (1972) 189.

[21] F. Jegerlehner and M. Y. Kalmykov, Nucl. Phys. B 676 (2004) 365 [arXiv:hep-ph/0308216];

F. Jegerlehner, M. Y. Kalmykov and O. Veretin, Nucl. Phys. B 658 (2003) 49 [arXiv:hep-ph/0212319;

F. Jegerlehner, M. Y. Kalmykov and O. Veretin, Nucl. Phys. B 641 (2002) 285 [arXiv:hep-ph/0105304];

J. Fleischer and F. Jegerlehner, Phys. Rev. D 23 (1981) 2001.

[22] G. Passarino and R. Pittau, Phys. Lett. B 228 (1989) 89.

[23] S. Eidelman and F. Jegerlehner, Z. Phys. C 67 (1995) 585 [arXiv hep-ph/9502298. F. Jegerlehner, arXiv hep-ph/0105283. F. Jegerlehner, arXiv hep-ph/0608329

[24] A. V. Kotikov, Phys. Lett. B 254 (1991) 158;

M. Caffo, H. Czyz, S. Laporta and E. Remiddi, Nuovo Cim. A 111 (1998) 365 [arXiv hep-th/9805118]. 